\title{
Synthesis of the Cationic Gallium Phthalocyanines and Their Catalytic Application in Gallium(III)-Activated Processes for Donor-Acceptor Substrates
}

Roman A. Novikov, ${ }^{*, a, c, \S}$ Anastasia A. Levina, ${ }^{a, \S}$ Denis D. Borisov, ${ }^{\mathrm{a},}$ Alexander D. Volodin, ${ }^{\mathrm{d}}$ Alexander A. Korlyukov, ${ }^{d}$ Yaroslav V. Tkachev, ${ }^{c}$ Yana B. Platonova, ${ }^{b}$ Larisa G. Tomilova, ${ }^{b}$ and Yury V. Tomilov*,a

${ }^{a}$ N. D. Zelinsky Institute of Organic Chemistry, Russian Academy of Sciences, 47 Leninsky prosp., 119991 Moscow, Russian Federation

${ }^{b}$ Department of Chemistry, Lomonosov Moscow State University, 1 Leninskie Gory, Moscow 119991, Russian Federation

${ }^{c}$ Engelhardt Institute of Molecular Biology, Russian Academy of Sciences, 32 Vavilov st., 119991 Moscow, Russian Federation

${ }^{d}$ Nesmeyanov Institute of Organoelement Compounds, Russian Academy of Sciences, Vavilov Str., 28, 119991 Moscow, Russian Federation

*e-mail: novikovfff@bk.ru

tom@ioc.ac.ru 
Table of contents:

General experimental details 3

General procedure for synthesis of gallium phthalocyanines 3

General procedure for synthesis of indenes 5

Synthesis of tetrahydronaphthalene 6

References 8

Spectral data and characterization of gallium phthalocyanines 9

(Micro)-photo of the phthalocyanine samples 9

SEM of gallium phthalocyanines 10

$\mathrm{UV} / \mathrm{Vis}$ 14

Crystal data for $n \mathrm{Bu}_{8} \mathrm{PcGaCl}$ 15

NMR spectra of gallium phthalocyanines 26

NMR spectra of indenes 51

NMR spectra of tetrahydronaphthalene 57 


\section{General experimental details}

All reagents used and solvents for chromatography were commercial-grade chemicals without additional purification. Styrylmalonates were synthesized from the corresponding D-A cyclopropanes according literature [1]. 1,2-dichloroethane as solvent for the reaction was distilled over $\mathrm{P}_{2} \mathrm{O}_{5}$. All operations with $\mathrm{AgSbF}_{6}$ and $\mathrm{GaCl}_{3}$ were carried out under dry argon atmosphere. TLC analysis was performed on Silufol chromatographic plates. For preparative chromatography, silica gel $60(0.040-0.063 \mathrm{~mm})$ was used. ${ }^{1} \mathrm{H},{ }^{13} \mathrm{C},{ }^{15} \mathrm{~N},{ }^{71} \mathrm{Ga}$ NMR spectra were recorded on a $300 \mathrm{MHz}(300.1,75.5,30.4$ and $91.5 \mathrm{MHz}$, respectively) and $400 \mathrm{MHz}(400.1$, 101.6, 40.1 and $122.0 \mathrm{MHz}$, respectively) spectrometers in $\mathrm{CDCl}_{3}, \mathrm{CD}_{2} \mathrm{Cl}_{2}, \mathrm{C}_{6} \mathrm{D}_{6}$ containing $0.05 \% \mathrm{Me}_{4} \mathrm{Si}$ as the internal standard. IR spectra were obtained on a Bruker IFS 113v. UV-Vis spectra registered on an Agilent $8453 \mathrm{UV}$-visible spectrophotometer using a $10 \mathrm{~mm}$ path length quartz cuvette. High-resolution mass spectra were obtained with a Bruker micrOTOF Instrument (ESI, positive or negative ion modes, capillary voltage $4500 \mathrm{~V}$ ).

Single crystals of $\mathrm{C}_{65} \mathrm{H}_{82} \mathrm{Cl}_{3} \mathrm{GaN}_{8}\left[n \mathrm{Bu}_{8} \mathrm{PcGaCl}\right]$ were recrystallized from $\mathrm{CH}_{2} \mathrm{Cl}_{2}$. A suitable crystal was selected and collected on a Bruker APEX-II CCD diffractometer. The crystal was kept at $120 \mathrm{~K}$ during data collection. Using Olex2 [2], the structure was solved with the ShelXT [3] structure solution program using Intrinsic Phasing and refined with the ShelXL [4] refinement package using Least Squares minimization. Crystallographic data for the gallium phthalocyanine $n \mathrm{Bu}_{8} \mathrm{PcGaCl}$ reported in this paper have been deposited in the Cambridge Crystallographic Data Centre as supplementary number CCDC 1979461.

Target-oriented approach was utilized for the optimization of the analytic measurements [5]. Before measurements the samples were mounted on a $25 \mathrm{~mm}$ aluminum specimen stub, fixed by conductive plasticine-like adhesive (powdered samples) or by conductive silver paint (films on aluminum foil) and coated with a thin film $(15 \mathrm{~nm})$ of carbon. The observations were carried out using Hitachi SU8000 field-emission scanning electron microscope (FE-SEM). Images were acquired in secondary electron mode at $10 \mathrm{kV}$ accelerating voltage. Morphology of the samples was studied taking into account possible influence of carbon coating on the surface. EDS-SEM studies were carried out using Oxford Instruments X-max 80 EDS system at $20 \mathrm{kV}$ accelerating voltage.

\section{General procedure for synthesis of gallium phthalocyanines}

The tube was charged with substituted phthalonitrile (4.00 equiv) then in inert atmosphere anhydrous gallium (III) chloride (1.05 equiv) was added, and finally DBU (2.5 equiv). Afterwards the tube was sealed and heated at $200{ }^{\circ} \mathrm{C}$ for $19 \mathrm{~h}$. The dark green residue was dissolved in $180 \mathrm{~mL} \mathrm{CHCl}_{3}$ and treated with an aqueous solution of $\mathrm{HCl}(2 \times 60 \mathrm{~mL}, 10 \%)$, then treated with distilled water $(2 \times 90 \mathrm{~mL})$. The organic layer was dried over $\mathrm{MgSO}_{4}$ and the solvent was removed in vacuo. 
Table S2. Solubility of gallium phtalocyanines in deuterated solvents for NMR:

\begin{tabular}{|l|l|l|}
\hline Deuterated solvent $(-0.5 \mathrm{~mL})$ & $\boldsymbol{t}$ Bu4 $4 \mathbf{P c G a C l}$ & $\boldsymbol{n B u} \mathbf{B P c G a C l}$ \\
\hline $\mathrm{CD}_{2} \mathrm{Cl}_{2}$ & $20-30 \mathrm{mg}$ & $10-15 \mathrm{mg}$ \\
\hline $\mathrm{C}_{6} \mathrm{D}_{6}$ & $5-10 \mathrm{mg}$ & $\sim 10 \mathrm{mg}$ \\
\hline $\mathrm{CD}_{3} \mathrm{CN}$ & insoluble & micellar \\
\hline DMSO-$d_{6}$ & poor soluble & micellar \\
\hline
\end{tabular}

\section{Chlorogallium(III) 2,(3)-tetra(tert-butyl)phtalocyanine ( $t$ Bu4PcGaCl)}

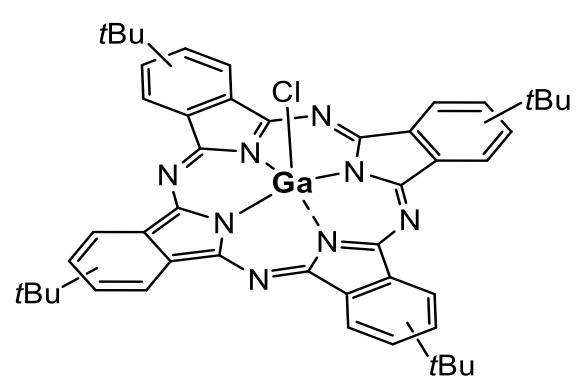

The title compound was prepared according to General Procedure using $2.21 \mathrm{~g}(12 \mathrm{mmol})$ of 4-(tertbutyl)phthalonitrile, $0.556 \mathrm{~g}(3.2 \mathrm{mmol})$ of anhydrous $\mathrm{GaCl}_{3}$, and $0.97 \mathrm{~g}(7.5 \mathrm{mmol})$ of DBU. After $19 \mathrm{~h} 200{ }^{\circ} \mathrm{C}$, workup, and removing the solvent in vacuo, the product was obtained in $2.3 \mathrm{~g}$ yield $(91 \%)$ as a blackish green with violet sparkle solid.

${ }^{1} \mathrm{H}$ NMR (300.13 MHz, $\mathrm{CD}_{2} \mathrm{Cl}_{2}$ ): $\delta$ 2.00, 2.03, 2.04, 2.06 and 2.07 (all s, 4:1:1:1:1, 36H, $t \mathrm{Bu}$ ), $8.20-8.48$ and $8.71-9.06$ (both $\mathrm{m}, 4+4 \mathrm{H}, \mathrm{H}_{\mathrm{Ar}}$ ), 8.87, 9.00, 9.28, 9.33, 9.41 and 9.44 (all $\mathrm{s}$, $\left.2: 2: 1: 1: 1: 1,4 \mathrm{H}, \mathrm{H}_{\mathrm{Ar}}\right)$.

${ }^{1} \mathrm{H}$ NMR (300.13 MHz, $\mathrm{C}_{6} \mathrm{D}_{6}$ ): $\delta$ 1.596, 1.602, 1.61, 1.63 and 1.66 (all s, 1:1:1:1:4, 4H, $\mathrm{H}_{\mathrm{Ar}}$ ), $8.08-8.21\left(\mathrm{~m}, 4 \mathrm{H}, \mathrm{H}_{\mathrm{Ar}}\right), 9.40-9.78$ (m, overlapped, $\left.8 \mathrm{H}, \mathrm{H}_{\mathrm{Ar}}\right)$.

${ }^{13} \mathrm{C}$ NMR (75.5 MHz, $\mathrm{CD}_{2} \mathrm{Cl}_{2}$ ): $\delta 31.9\left(\right.$ all $\left.\mathrm{CH}_{3}\right), 36.2$ (all $\mathrm{C}_{t \mathrm{Bu}}$ ), $118.0-120.3,121.3-124.0$ and 128.9 (all $\mathrm{CH}_{\mathrm{Ar}}$ ), 132.4 - 134.8, $134.8-137.6,149.2-153.0$ and $153.0-156.0$ (all C).

${ }^{71} \mathrm{Ga}$ NMR (91.5 MHz): $\delta+114 \mathrm{ppm}\left(\mathrm{W}_{1 / 2}=2200 \mathrm{~Hz}\right)$.

IR (KBr): $\tilde{v}$ 2959, 2904, 2865, 1615, 1507, 1484, 1394, 1365, 1335, 1282, 1258, 1202, 1156, $1088,1052,930,895,831,765,750,695,670,601,532 \mathrm{~cm}^{-1}$.

$\mathrm{UV} / \mathrm{Vis}\left(\mathrm{CHCl}_{3}\right): \lambda 695,665,626,358 \mathrm{~nm}$.

HRMS calcd for $\mathrm{C}_{48} \mathrm{H}_{48} \mathrm{ClGaN}_{8}(M): M+H, 841.3019$. Found: $m / z$ 841.3013.

\section{Chlorogallium(III) 2,3-octa( $n$-butyl)phtalocyanine ( $n$ BusPcGaCl)}

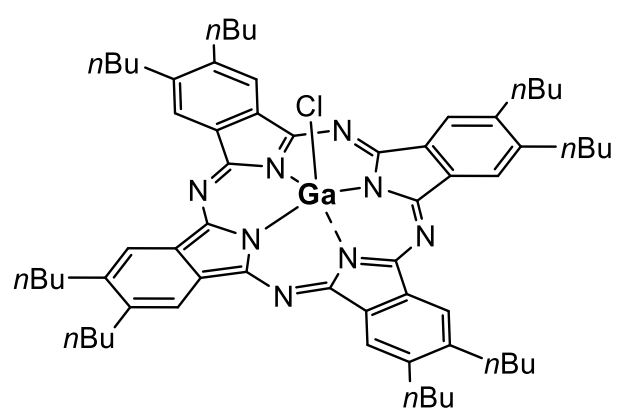

The title compound was prepared according to General Procedure using $2.21 \mathrm{~g}$ (9.2 mmol) of 4,5-dibutylphthalonitrile, $0.425 \mathrm{~g}(2.4 \mathrm{mmol})$ of anhydrous $\mathrm{GaCl}_{3}$, and $0.74 \mathrm{~g}(5.8 \mathrm{mmol})$ of DBU. After $19 \mathrm{~h} 200{ }^{\circ} \mathrm{C}$, workup, and removing the solvent in vacuo, the product was obtained in $2.10 \mathrm{~g}$ yield $(81 \%)$ as a blackish green solid. The compound can be recrystallized from a mixture of $\mathrm{CH}_{2} \mathrm{Cl}_{2} / \mathrm{MeOH}(\mathrm{v} / \mathrm{v} 4: 3)$ in $1.05 \mathrm{~g}$ yield

$(50 \%)$ as green with a blue tint microcrystals.

${ }^{1} \mathrm{H}$ NMR (300.13 MHz, $\left.\mathrm{CD}_{2} \mathrm{Cl}_{2}\right): \delta 1.29\left(\mathrm{t}, 24 \mathrm{H}, \mathrm{CH}_{3},{ }^{3} \mathrm{~J}=7.3 \mathrm{~Hz}\right), 1.70-2.97$ and $1.97-2.21$ and $3.07-3.42$ (all $\left.\mathrm{m}, 48 \mathrm{H}, \mathrm{CH}_{2}\right), 8.73\left(\mathrm{~s}, 8 \mathrm{H}, \mathrm{H}_{\mathrm{Ar}}\right)$.

${ }^{1} \mathrm{H}$ NMR (300.13 MHz, $\left.\mathrm{C}_{6} \mathrm{D}_{6}\right): \delta 1.07\left(\mathrm{t}, 24 \mathrm{H}, \mathrm{CH}_{3},{ }^{3} \mathrm{~J}=7.3 \mathrm{~Hz}\right), 1.42-1.71$ and $1.74-1.95$ and $2.89-3.18$ (all m, 48H, $\mathrm{CH}_{2}$ ), $9.17\left(\mathrm{~s}, 8 \mathrm{H}, \mathrm{H}_{\mathrm{Ar}}\right.$ ). 
${ }^{13} \mathrm{C}$ NMR (75.5 MHz, $\left.\mathrm{CD}_{2} \mathrm{Cl}_{2}\right): \delta 14.02\left(\right.$ all $\left.\mathrm{CH}_{3}\right), 23.4,33.8$ and $33.9\left(\right.$ all $\left.\mathrm{CH}_{2}\right), 122.5\left(\right.$ all $\left.\mathrm{CH}_{\mathrm{Ar}}\right)$, 134.1, 144.5 and 151.1 (all C).

${ }^{71} \mathrm{Ga}$ NMR (91.5 MHz): $\delta+115 \mathrm{ppm}\left(\mathrm{W}_{1 / 2}=4500 \mathrm{~Hz}\right)$.

IR (KBr): $\tilde{v}$ 2956, 2927, 2870, 1619, 1506, 1456, 1414, 1377, 1370, 1340, 1301, 1169, 1098, $1076,1029,872,748,461 \mathrm{~cm}^{-1}$.

$\mathrm{UV} / \mathrm{V}$ is $\left(\mathrm{CHCl}_{3}\right): \lambda 704,672,634,355 \mathrm{~nm}$.

HRMS calcd for $\mathrm{C}_{48} \mathrm{H}_{48} \mathrm{ClGaN}_{8}(M): M-C l, 1029.5756$. Found: $m / z$ 1029.5725.

Crystal Data for $\mathrm{C}_{65} \mathrm{H}_{82} \mathrm{Cl}_{3} \mathrm{GaN}_{8}$ see p. S15.

\section{General procedure for synthesis of indenes}

Under an argon atmosphere the Schlenk flask was charged with $0.012 \mathrm{mmol}_{\text {of }} \mathrm{AgSbF}_{6}, 0.019$ mmol of gallium phthalocyanine, a mixture of $0.24 \mathrm{mmol}$ of styrylmalonate, $0.48 \mathrm{mmol}$ of aromatic aldehyde, $3 \mathrm{~mL}$ DCE, and a magnetic stir bar. The reaction was heated at reflux for $3 \mathrm{~h}$. Then at room temperature an aqueous solution of $\mathrm{HCl}(10 \mathrm{~mL}, 10 \%)$ was added until $p \mathrm{H} 3$ achieved, the reaction mixture was extracted with $\mathrm{CH}_{2} \mathrm{Cl}_{2}(3 \times 10 \mathrm{~mL})$. A catalytic amount of $\mathrm{HBF}_{4}(50 \%$ aq.) and an excess of ethereal solution of diazomethane were added to the organic layer, an argon was bubbled through the solution and then it was dried over $\mathrm{MgSO}_{4}$ and the solvent was removed in vacuo. The residue was purified by column chromatography on silica gel (benzene to benzene-ethyl acetate, 20:1) to afford the product.

\section{Dimethyl 2-(2-phenyl-1H-inden-1-yl)malonate}

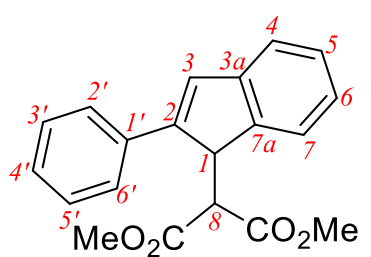

The title compound was prepared from $4.0 \mathrm{mg}(0.012 \mathrm{mmol})$ of $\mathrm{AgSbF}_{6}, 16 \mathrm{mg}(0.019 \mathrm{mmol})$ of $t \mathrm{Bu}_{4} \mathrm{PcGaCl}, 56 \mathrm{mg}(0.24 \mathrm{mmol})$ of the dimethyl 2-styrylmalonate, and $51 \mathrm{mg}(0.48 \mathrm{mmol})$ of the benzaldehyde in yield $58 \mathrm{mg}(75 \%)$ as colorless oil.

${ }^{1} \mathrm{H}$ NMR $\left(300.13 \mathrm{MHz}, \mathrm{CDCl}_{3}\right): \delta 3.34(\mathrm{~s}, 3 \mathrm{H}, \mathrm{OMe}), 3.73(\mathrm{~s}, 3 \mathrm{H}$, OMe), $3.91\left(\mathrm{~d}, 1 \mathrm{H}, \mathrm{H}(8),{ }^{3} \mathrm{~J}=4.0 \mathrm{~Hz}\right), 4.70$ (d, 1H, H(1), $\left.{ }^{3} \mathrm{~J}=4.0 \mathrm{~Hz}\right), 7.07$ (d, 1H, H(3), ${ }^{4} \mathrm{~J}=$ $0.9 \mathrm{~Hz}), 7.21\left(\mathrm{td}, 1 \mathrm{H}, \mathrm{H}(6),{ }^{3} J=7.4 \mathrm{~Hz},{ }^{4} J=1.3 \mathrm{~Hz}\right), 7.26-7.53\left(\mathrm{~m}, 7 \mathrm{H}, \mathrm{H}_{\mathrm{Ar}}\right), 7.58(\mathrm{~d}, 1 \mathrm{H}, \mathrm{H}(7)$, $\left.{ }^{3} J=7.4 \mathrm{~Hz}\right)$.

${ }^{13} \mathrm{C}$ NMR (75.5 MHz, $\left.\mathrm{CDCl}_{3}\right): \delta 47.9(\mathrm{C}(1)), 51.8(\mathrm{OMe}), 52.5(\mathrm{OMe}), 52.6(\mathrm{C}(8)), 121.2(\mathrm{C}(4))$, $124.5(\mathrm{C}(7)), 125.2(\mathrm{C}(6)), 127.0\left(\mathrm{C}\left(2^{\prime}\right), \mathrm{C}\left(6^{\prime}\right)\right), 127.6\left(\mathrm{C}\left(4^{\prime}\right)\right), 127.7(\mathrm{C}(5)), 128.8\left(\mathrm{C}\left(3^{\prime}\right), \mathrm{C}\left(5^{\prime}\right)\right)$, $129.1(\mathrm{C}(3)), 135.0\left(\mathrm{C}\left(1^{\prime}\right)\right), 143.8(\mathrm{C}(7 \mathrm{a})), 144.1(\mathrm{C}(3 \mathrm{a})), 148.3(\mathrm{C}(2)), 167.2$ and 169.3 (2 COO).

NMR spectra are as described in the literature [6].

\section{Dimethyl 2-(2-(3-bromophenyl)-1H-inden-1-yl)malonate}

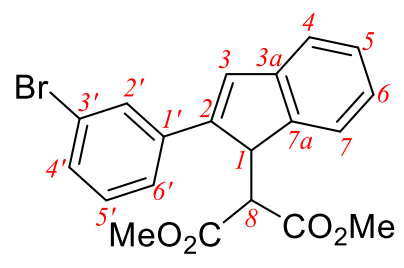

The title compound was prepared from $4.0 \mathrm{mg}(0.012 \mathrm{mmol})$ of $\mathrm{AgSbF}_{6}, 16 \mathrm{mg}(0.019 \mathrm{mmol})$ of $t \mathrm{Bu}_{4} \mathrm{PcGaCl}, 75 \mathrm{mg}(0.24 \mathrm{mmol})$ of the dimethyl 2-(3-bromostyryl)malonate, and $51 \mathrm{mg}(0.48$ $\mathrm{mmol})$ of the benzaldehyde in yield $66 \mathrm{mg}(69 \%)$ as colorless oil.

${ }^{1} \mathrm{H}$ NMR (400.1 MHz, $\left.\mathrm{CDCl}_{3}\right): \delta 3.34$ (s, 3H, OMe), 3.71 (s, 3H, 
OMe), 3.85 (d, 1H, H(8), $J=3.9 \mathrm{~Hz}$ ), 4.62 (br.d, $\left.1 \mathrm{H}, \mathrm{H}(1),{ }^{3} J=3.9 \mathrm{~Hz}\right), 7.06$ (d, 1H, H(3), $J=$ $1.0 \mathrm{~Hz}$ ), 7.16-7.48 (m, 6H, $\left.\mathrm{H}_{\mathrm{Ar}}\right), 7.54$ (br.d, $\left.1 \mathrm{H}, \mathrm{H}_{\mathrm{Ar}},{ }^{3} J=7.5 \mathrm{~Hz}\right), 7.61$ (t, $1 \mathrm{H}, \mathrm{H}\left(2^{\prime}\right),{ }^{4} J=1.8$ $\mathrm{Hz})$.

${ }^{13} \mathrm{C}$ NMR (100.6 MHz, $\left.\mathrm{CDCl}_{3}\right): \delta 48.0(\mathrm{C}(1)), 52.0(\mathrm{OMe}), 52.6(\mathrm{C}(8)), 52.7(\mathrm{OMe}), 121.6$, 124.7, 125.7, 127.8, 130.0, 130.4, 130.6 and 130.7 (8 $\left.\mathrm{CH}_{\mathrm{Ar}}\right), 123.1\left(\mathrm{C}\left(3^{\prime}\right)\right), 137.4,143.7,143.9$ and 146.9 ( $\left.4 \mathrm{C}_{\mathrm{Ar}}\right), 167.2$ and 169.1 (2 COO).

NMR spectra are as described in the literature [6].

\section{Dimethyl 2-(8-phenyl-7H-cyclopenta[a]pyren-7-yl)malonate}

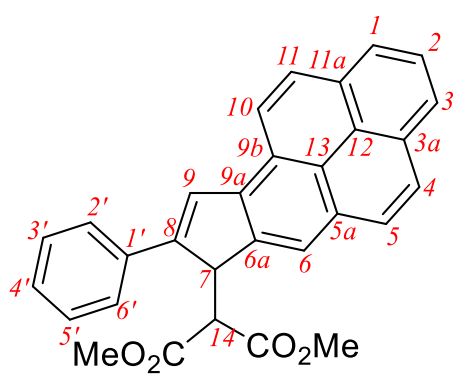

The title compound was prepared from $4.0 \mathrm{mg}(0.012 \mathrm{mmol})$ of $\mathrm{AgSbF}_{6}, 16 \mathrm{mg}(0.019 \mathrm{mmol})$ of $t \mathrm{Bu}_{4} \mathrm{PcGaCl}, 56 \mathrm{mg}(0.24$ $\mathrm{mmol})$ of the dimethyl 2-styrylmalonate, and $223 \mathrm{mg}(0.48$ $\mathrm{mmol}$ ) of the 1-pyrenecarboxaldehyde in yield $68 \mathrm{mg}(63 \%)$ as dark oil.

${ }^{1} \mathrm{H}$ NMR (400.1 MHz, $\left.\mathrm{CDCl}_{3}\right): \delta 3.16$ (s, 3H, OMe), 3.77 (s, $3 \mathrm{H}, \mathrm{OMe}), 4.06$ (d, $\left.1 \mathrm{H}, \mathrm{CH},{ }^{3} \mathrm{~J}=3.9 \mathrm{~Hz}\right), 4.97$ (br.d, 1H, H(7), ${ }^{3} J=3.9 \mathrm{~Hz}$ ), 7.35 (br.t, $1 \mathrm{H}, \mathrm{H}\left(4^{\prime}\right),{ }^{3} J=7.5 \mathrm{~Hz}$ ), 7.47 (br.dd, $2 \mathrm{H}, \mathrm{H}\left(3^{\prime}\right)$ and $\mathrm{H}\left(5^{\prime}\right),{ }^{3} J=7.5$ and $8.4 \mathrm{~Hz}$ ), 7.59 (dd, $2 \mathrm{H}, \mathrm{H}\left(2^{\prime}\right)$ and $\mathrm{H}\left(6^{\prime}\right),{ }^{3} J=8.4,{ }^{4} J=1.1 \mathrm{~Hz}$ ), 7.83 (br.s, $1 \mathrm{H}, \mathrm{H}(9)$ ), 7.94 (t, $1 \mathrm{H}$, $\left.\mathrm{H}(2),{ }^{3} \mathrm{~J}=7.6 \mathrm{~Hz}\right), 8.00\left(\mathrm{~d}, 1 \mathrm{H}, \mathrm{H}(4),{ }^{3} \mathrm{~J}=8.9 \mathrm{~Hz}\right), 8.06\left(\mathrm{~d}, 1 \mathrm{H}, \mathrm{H}(11),{ }^{3} \mathrm{~J}=9.0 \mathrm{~Hz}\right), 8.07(\mathrm{~d}, 1 \mathrm{H}$, $\left.\mathrm{H}(5),{ }^{3} \mathrm{~J}=8.9 \mathrm{~Hz}\right), 8.12$ (br.t, $2 \mathrm{H}, \mathrm{H}(1)$ and $\left.\mathrm{H}(3),{ }^{3} \mathrm{~J}=6.8 \mathrm{~Hz}\right), 8.30$ (d, $1 \mathrm{H}, \mathrm{H}(10),{ }^{3} \mathrm{~J}=9.0 \mathrm{~Hz}$ ), 8.35 (br.s, $1 \mathrm{H}, \mathrm{H}(6))$.

${ }^{13} \mathrm{C}$ NMR (100.6 MHz, $\left.\mathrm{CDCl}_{3}\right): \delta 48.4(\mathrm{C}(7)), 51.9$ and $52.8(2 \mathrm{OMe}), 53.0(\mathrm{C}(14)), 121.5(\mathrm{C}(6))$, 123.6 (C(10)), 124.0 (C(11a)), 124.6 (C(1)), 124.7 (C(13)), 124.9 (C(3)), 125.3 (C(12)), 125.7 $(\mathrm{C}(2)), 126.5(\mathrm{C}(4)), 127.0(\mathrm{C}(9)), 127.3(\mathrm{C}(11)), 127.4\left(\mathrm{C}\left(2^{\prime}\right)\right.$ and $\left.\mathrm{C}\left(6^{\prime}\right)\right), 128.0\left(\mathrm{C}\left(4^{\prime}\right)\right), 128.3$ $(\mathrm{C}(5)), 129.0\left(\mathrm{C}\left(3^{\prime}\right)\right.$ and $\left.\mathrm{C}\left(5^{\prime}\right)\right), 129.3(\mathrm{C}(3 \mathrm{a})), 131.3(\mathrm{C}(9 \mathrm{~b})), 131.5(\mathrm{C}(5 \mathrm{a})), 135.2\left(\mathrm{C}\left(1^{\prime}\right)\right), 139.2$ (C(9a)), 141.8 (C(6a)), 149.6 (C(8)), 167.3 and 169.6 (2 COO).

NMR spectra are as described in the literature [6].

\section{Synthesis of tetrahydronaphthalene}

\section{Dimethyl 2-(4-phenyl-1,2,3,4-tetrahydronaphthalen-2-yl)malonate}

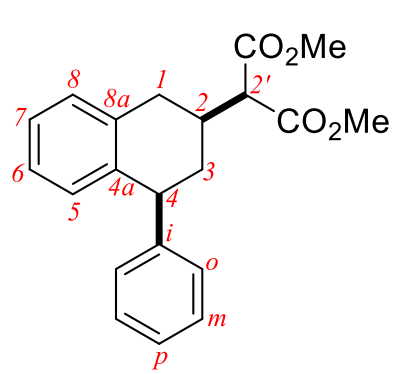

Under an argon atmosphere the Schlenk flask was charged with 4.0 $\mathrm{mg}(0.012 \mathrm{mmol})$ of $\mathrm{AgSbF}_{6}, 16 \mathrm{mg}(0.019 \mathrm{mmol})$ of $t \mathrm{Bu}_{4} \mathrm{PcGaCl}$, a mixture of $56 \mathrm{mg}(0.24 \mathrm{mmol})$ of the dimethyl 2-styrylmalonate and $200 \mathrm{mg}$ (1.92 $\mathrm{mmol})$ of styrene in $3 \mathrm{~mL} \mathrm{DCE}$, and a magnetic stir bar. The reaction was heated at reflux for $3 \mathrm{~h}$. Then the mixture was diluted with $15 \mathrm{~mL} \mathrm{CH} \mathrm{Cl}_{2}$ and treated with an aqueous solution of $\mathrm{HCl}(10 \mathrm{~mL}, 10 \%)$, the organic layer was separated and the aqueous layer was extracted with $\mathrm{CH}_{2} \mathrm{Cl}_{2}(3 \times 10 \mathrm{~mL})$. The combined organic extracts were dried over $\mathrm{MgSO}_{4}$ and the solvent was removed in vacuo. The residue was purified by column chromatography on silica gel (benzene-ethyl acetate, from 50:1 to 20:1) to afford the product in yield $30 \mathrm{mg}(37 \%, \mathrm{dr} \sim 3.8 / 1)$ as colorless oil. 
${ }^{1} \mathrm{H}$ NMR (400.1 MHz, $\mathrm{CDCl}_{3}$ ): $\delta 1.67$ (ddd, $1 \mathrm{H}, \mathrm{H}(3),{ }^{2} J=12.6 \mathrm{~Hz},{ }^{3} J=12.3$ and $12.1 \mathrm{~Hz}$ ), 2.17 (dddd, $1 \mathrm{H}, \mathrm{H}(3),{ }^{2} J=12.6 \mathrm{~Hz},{ }^{3} J=5.4$ and $2.6 \mathrm{~Hz},{ }^{4} J=2.2 \mathrm{~Hz}$ ), $2.68-2.76(\mathrm{~m}, 1 \mathrm{H}, \mathrm{H}(2)$ ), 2.82 $\left(\mathrm{dd}, 1 \mathrm{H}, \mathrm{H}(1),{ }^{2} J=15.5 \mathrm{~Hz},{ }^{3} J=11.7 \mathrm{~Hz}\right), 2.93\left(\mathrm{ddd}, 1 \mathrm{H}, \mathrm{H}(1),{ }^{2} J=15.5 \mathrm{~Hz},{ }^{3} J=4.2 \mathrm{~Hz},{ }^{4} J=\right.$ $2.2 \mathrm{~Hz}$ ), 3.38 (d, 1H, H(2'), ${ }^{3} J=8.4 \mathrm{~Hz}$ ), 3.72 (s, 3H, OMe), 3.77 (s, 3H, OMe), 4.12 (dd, 1H, $\mathrm{H}(4),{ }^{3} \mathrm{~J}=12.1$ and $\left.5.4 \mathrm{~Hz}\right), 6.75$ (br.d, $\left.1 \mathrm{H}, \mathrm{H}(5),{ }^{3} \mathrm{~J}=7.6 \mathrm{~Hz}\right), 7.00(\mathrm{~m}, 1 \mathrm{H}, \mathrm{H}(6)), 7.07$ (m, 1H, $\mathrm{H}(7)), 7.08(\mathrm{~m}, 1 \mathrm{H}, \mathrm{H}(8)), 7.16(\mathrm{~m}, 2 \mathrm{H}, 2 o-\mathrm{H}), 7.24(\mathrm{~m}, 1 \mathrm{H}, p-\mathrm{H}), 7.29(\mathrm{~m}, 2 \mathrm{H}, 2 m-\mathrm{H})$.

${ }^{13} \mathrm{C}$ NMR (100.6 MHz, $\left.\mathrm{CDCl}_{3}\right) \delta 34.3(\mathrm{C}(1)), 35.1(\mathrm{C}(2)), 38.4(\mathrm{C}(3)), 46.9(\mathrm{C}(4)), 52.51$ and $52.54(2 \mathrm{OMe}), 57.4\left(\mathrm{C}\left(2^{\prime}\right)\right), 126.1(\mathrm{C}(6)$ and $\mathrm{C}(8)), 126.5(\mathrm{C}(p)), 128.6(2 \mathrm{C}(m)), 128.86(2$ $\mathrm{C}(o)), 128.90(\mathrm{C}(7)), 129.5(\mathrm{C}(5)), 135.7(\mathrm{C}(8 \mathrm{a})), 139.3(\mathrm{C}(4 \mathrm{a})), 146.4(\mathrm{C}(\mathrm{i})), 168.7$ and 168.8 (2COO).

minor isomer: ${ }^{1} \mathrm{H}$ NMR $\left(400.1 \mathrm{MHz}, \mathrm{CDCl}_{3}\right) \delta 1.96-2.05(\mathrm{~m}, 2 \mathrm{H}, \mathrm{H}(3)), 2.60-2.67(\mathrm{~m}, 1 \mathrm{H}$, $\left.\mathrm{H}(2),{ }^{3} J=9.8,8.4,4.6 \mathrm{~Hz}\right), 2.72\left(\mathrm{dd}, 1 \mathrm{H}, \mathrm{H}(1),{ }^{2} J=16.2 \mathrm{~Hz},{ }^{3} J=9.8 \mathrm{~Hz}\right), 3.03\left(\mathrm{dd}, 1 \mathrm{H}, \mathrm{H}(1),{ }^{2} J\right.$ $\left.=16.2 \mathrm{~Hz},{ }^{3} \mathrm{~J}=4.6 \mathrm{~Hz}\right), 3.36\left(\mathrm{~d}, 1 \mathrm{H}, \mathrm{H}\left(2^{\prime}\right),{ }^{3} \mathrm{~J}=8.4 \mathrm{~Hz}\right), 3.65$ (s, 3H, OMe), 3.69 (s, 3H, OMe), $4.29\left(\mathrm{dd}, 1 \mathrm{H}, \mathrm{H}(4),{ }^{3} \mathrm{~J}=5.3\right.$ and $\left.5.1 \mathrm{~Hz}\right), 6.94\left(\mathrm{br} . \mathrm{d}, 1 \mathrm{H}, \mathrm{H}(5),{ }^{3} \mathrm{~J}=7.6 \mathrm{~Hz}\right), 7.00(\mathrm{~m}, 2 \mathrm{H}, 2 o-\mathrm{H})$, $7.11(\mathrm{~m}, 1 \mathrm{H}, \mathrm{H}(6)), 7.14(\mathrm{~m}, 1 \mathrm{H}, \mathrm{H}(8)), 7.15$ (m, 1H, H(7)), 7.17 (m, 1H, p-H), 7.25 (m, 2H, 2 $m-\mathrm{H})$.

${ }^{13} \mathrm{C}$ NMR (100.6 MHz, $\left.\mathrm{CDCl}_{3}\right) \delta 30.2(\mathrm{C}(2)), 32.3(\mathrm{C}(1)), 36.0(\mathrm{C}(3)), 43.6(\mathrm{C}(4)), 52.35$ and 52.38 (2 OMe), $56.3\left(\mathrm{C}\left(2^{\prime}\right)\right), 126.1(\mathrm{C}(6)), 126.2(\mathrm{C}(p)), 126.4(\mathrm{C}(8)), 128.2(2 \mathrm{C}(m)), 128.7$ (2 $\mathrm{C}(o)), 129.2(\mathrm{C}(7)), 130.4(\mathrm{C}(5)), 136.0(\mathrm{C}(8 \mathrm{a})), 137.6(\mathrm{C}(4 \mathrm{a})), 146.6(\mathrm{C}(i)), 168.7$ and 168.9 (2COO).

NMR spectra are as described in the literature [7]. 


\section{References}

1. Novikov, R. A., Tarasova, A. V., Korolev, V. A., Timofeev, V. P., Tomilov, Y. V. (2014). Angew. Chem. Int. Ed. 53, 3187-3191.

2. Dolomanov, O.V., Bourhis, L.J., Gildea, R.J, Howard, J.A.K. \& Puschmann, H. (2009), J. Appl. Cryst. 42, 339-341.

3. Sheldrick, G.M. (2015). Acta Cryst. A71, 3-8.

4. Sheldrick, G.M. (2015). Acta Cryst. C71, 3-8.

5. Kachala, V. V., Khemchyan, L. L., Kashin, A. S., Orlov, N. V., Grachev, A. A., Zalesskiy, S. S., Ananikov, V. P. (2013). Russ. Chem. Rev., 82, 648-685.

6. Borisov D. D., Novikov R. A., Tomilov Y. V. (2016). Angew. Chem. Int. Ed., 55 (40), 1223312237.

7. Novikov R. A., Tarasova A. V., Korolev V. A., Shulishov E. V., Timofeev V. P., Tomilov Y. V. (2015). J. Org. Chem., 80 (16), 8225-8235. 
Spectral data and characterization of gallium phthalocyanines

Figure S10. (Micro)-photo of the phthalocyanine samples $t \mathrm{Bu}{ }_{4} \mathrm{PcGaCl}$

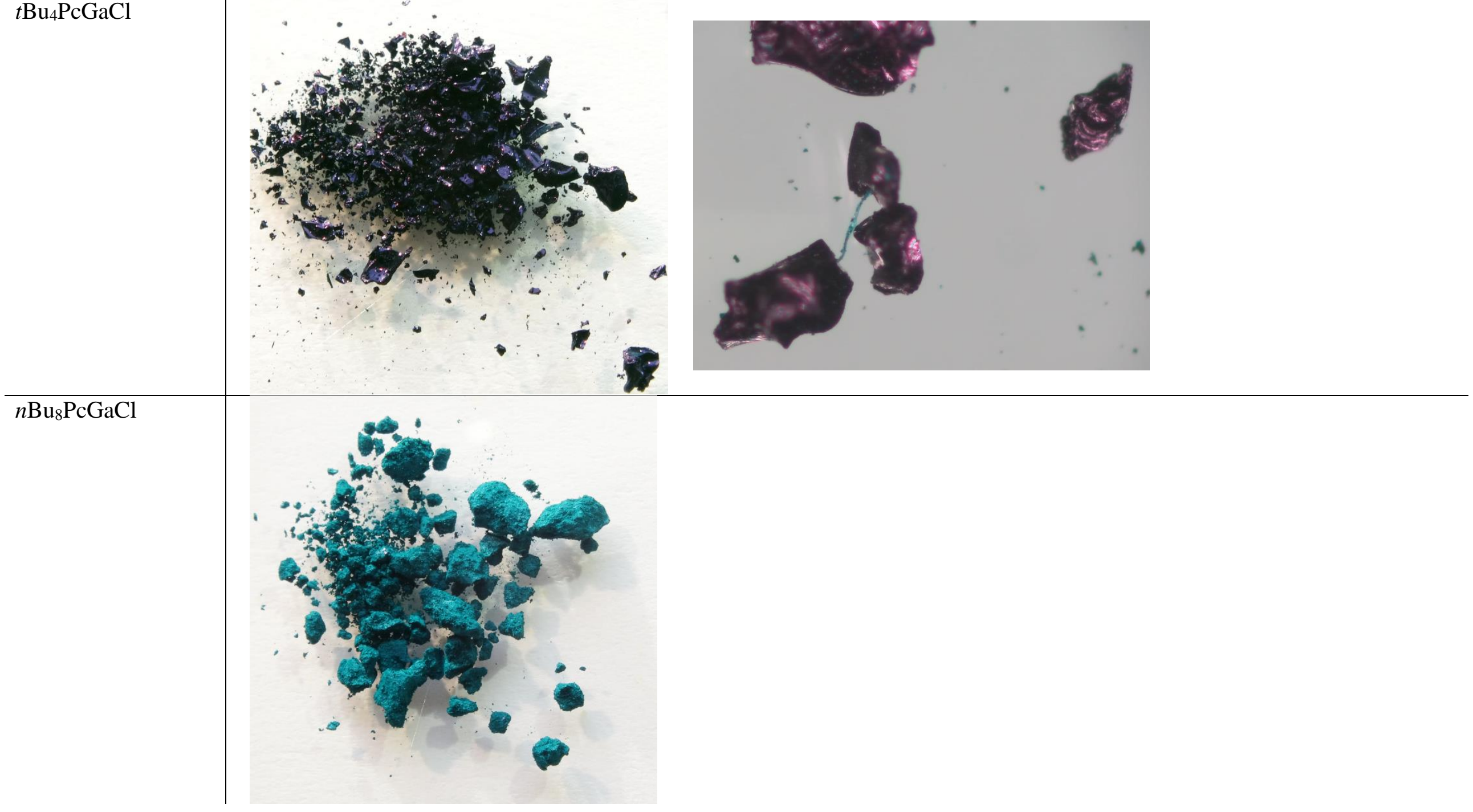


Figure S11. $t \mathrm{Bu}_{4} \mathrm{PcGaCl}$ (film)
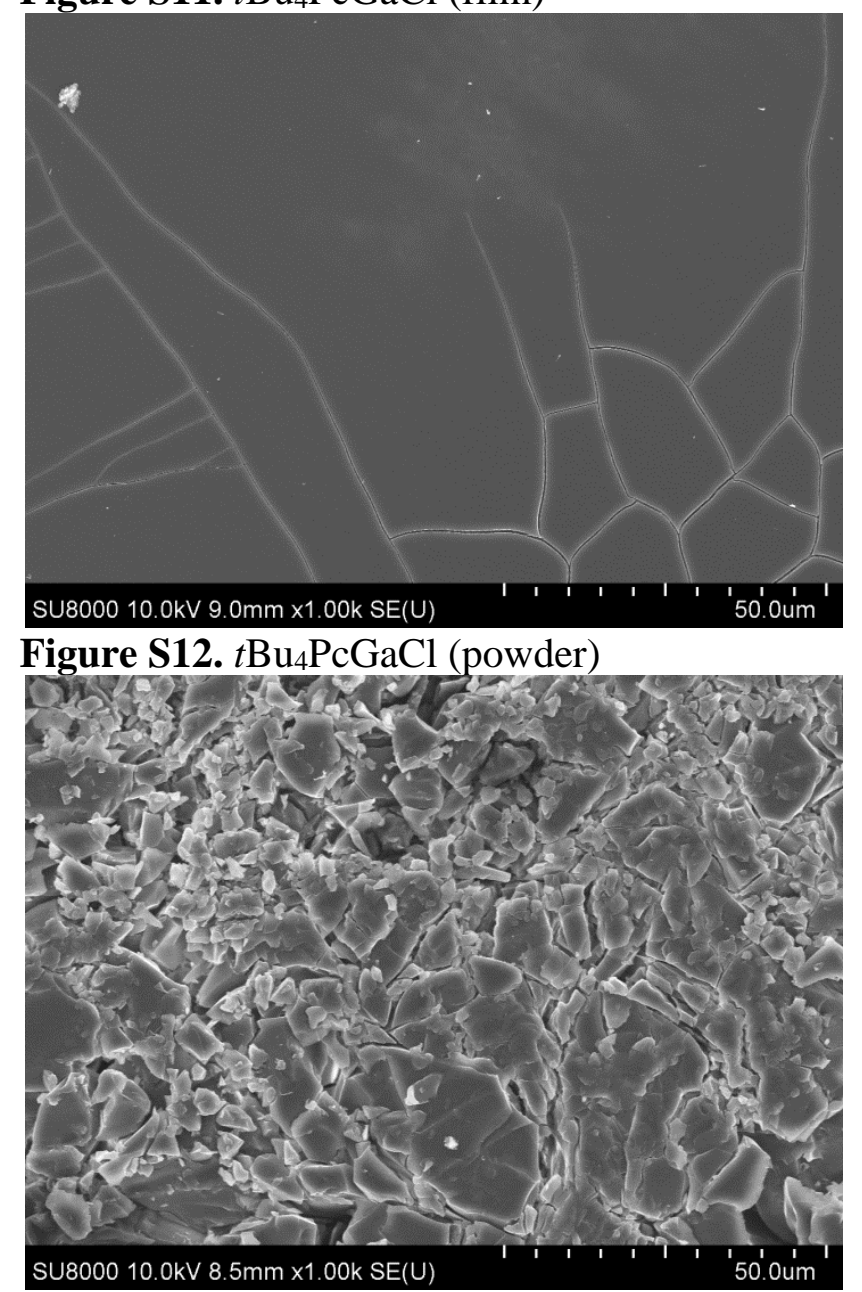
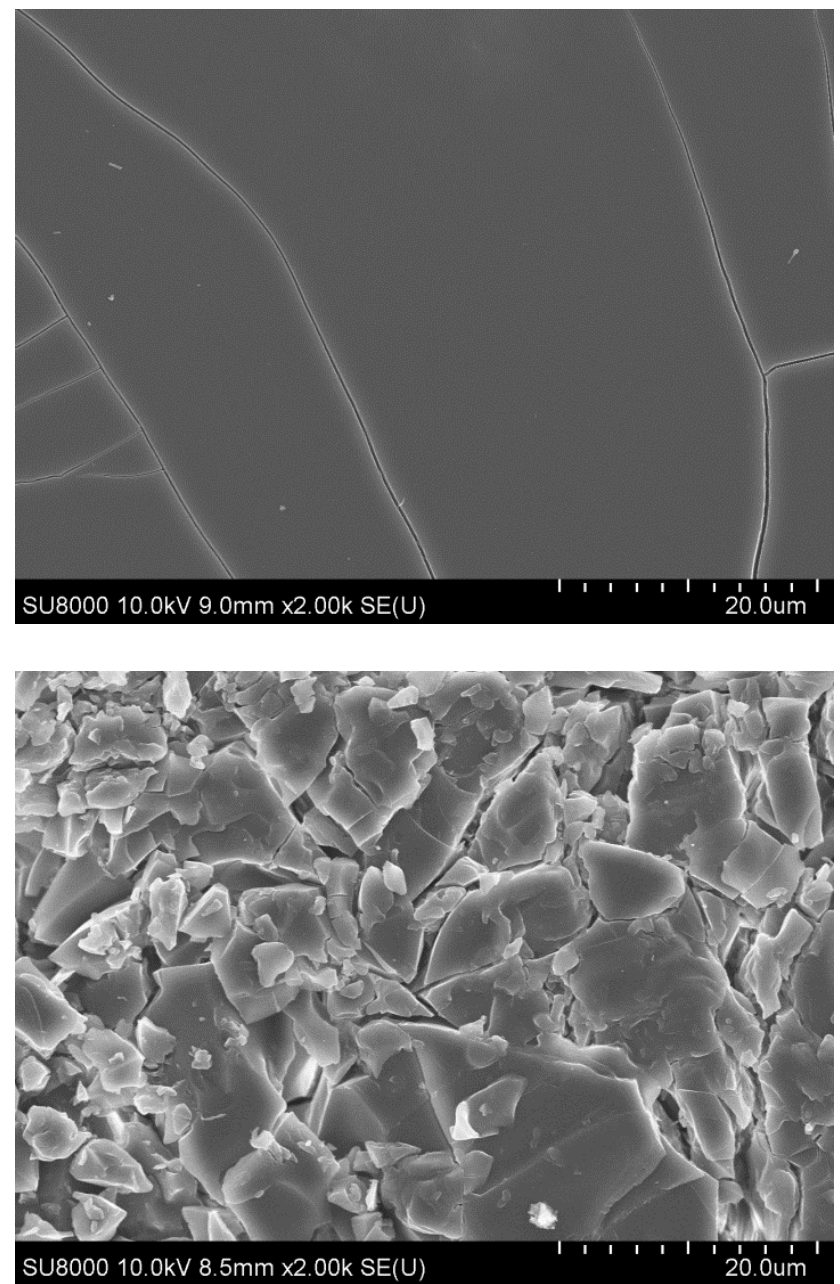
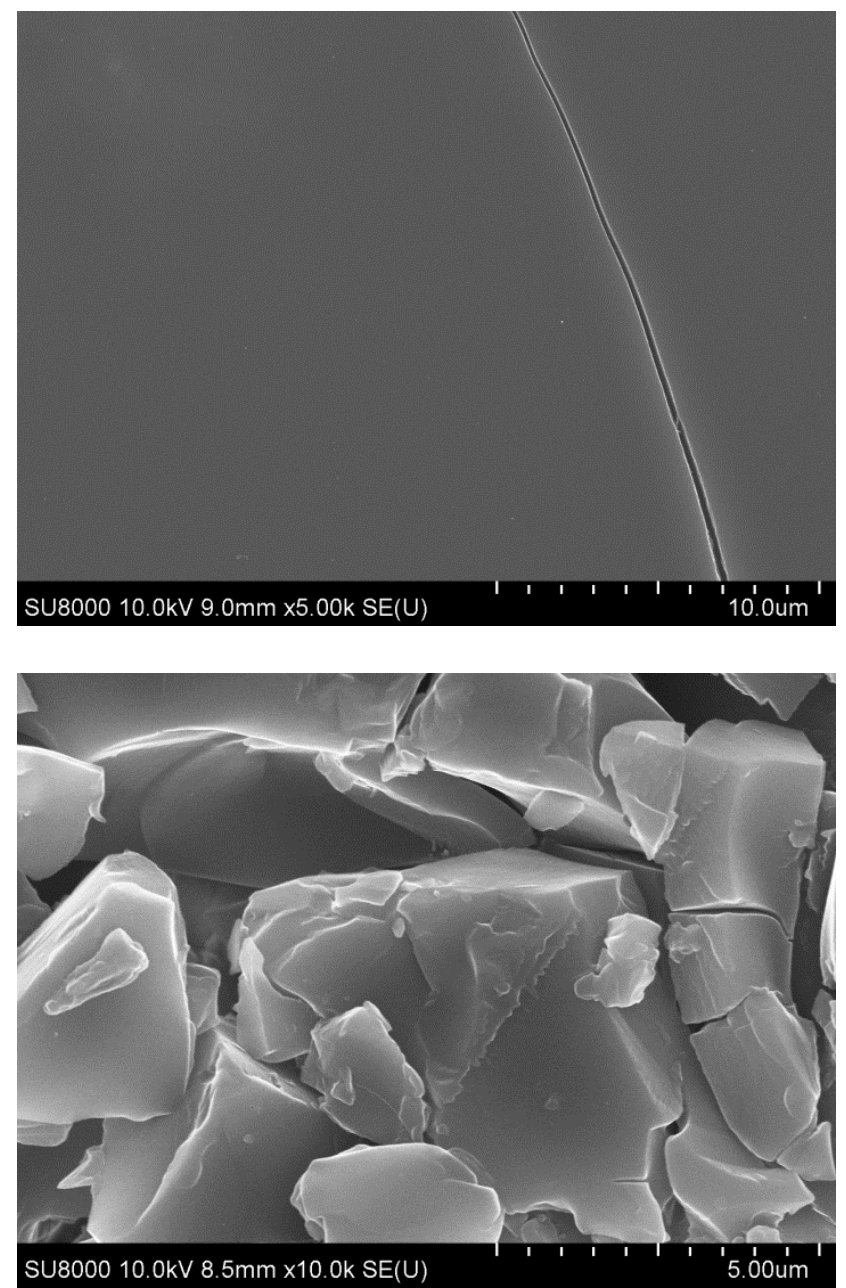


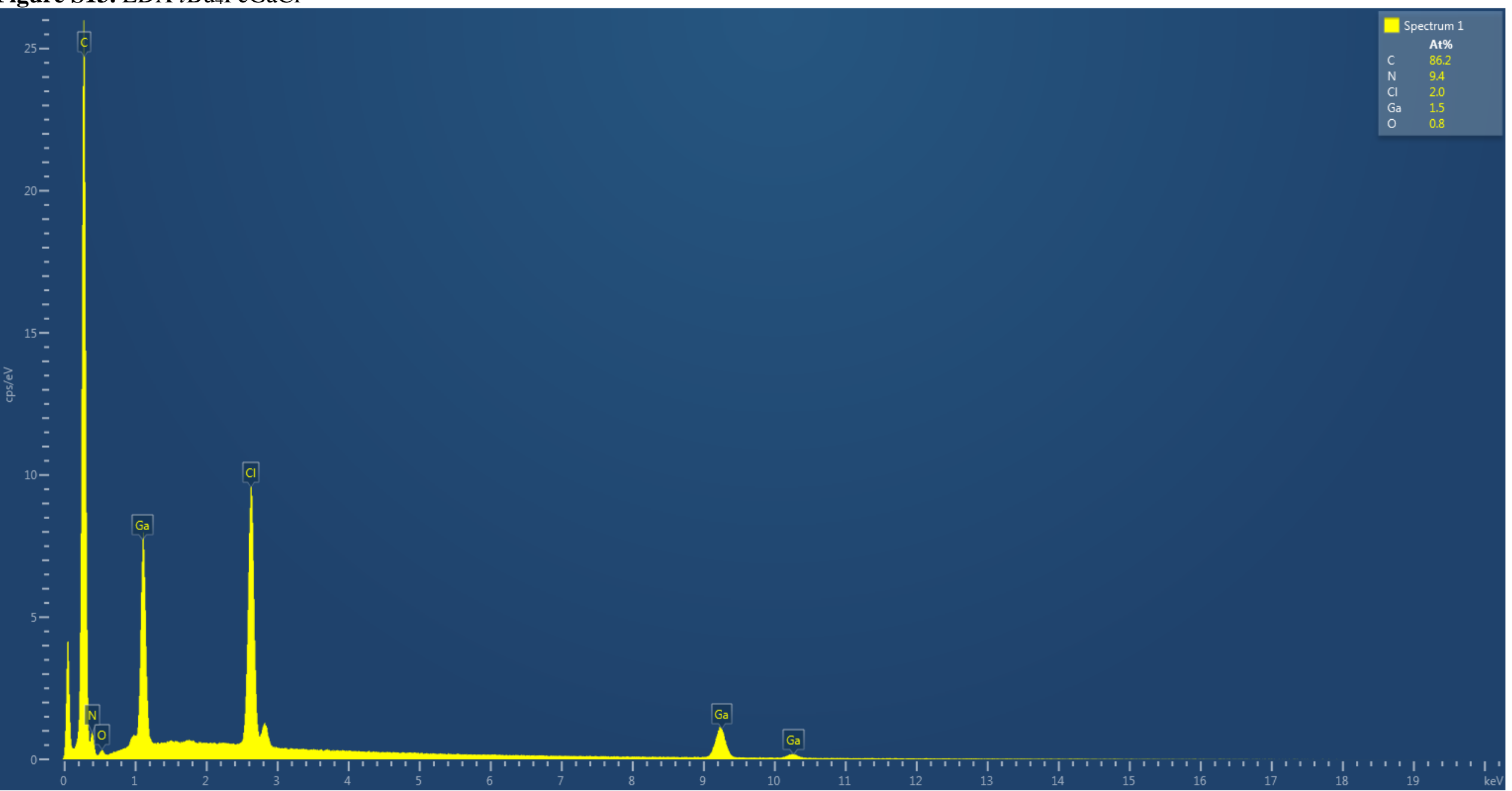


Figure S14. $n \mathrm{Bu}_{8} \mathrm{PcGaCl}$ (film)
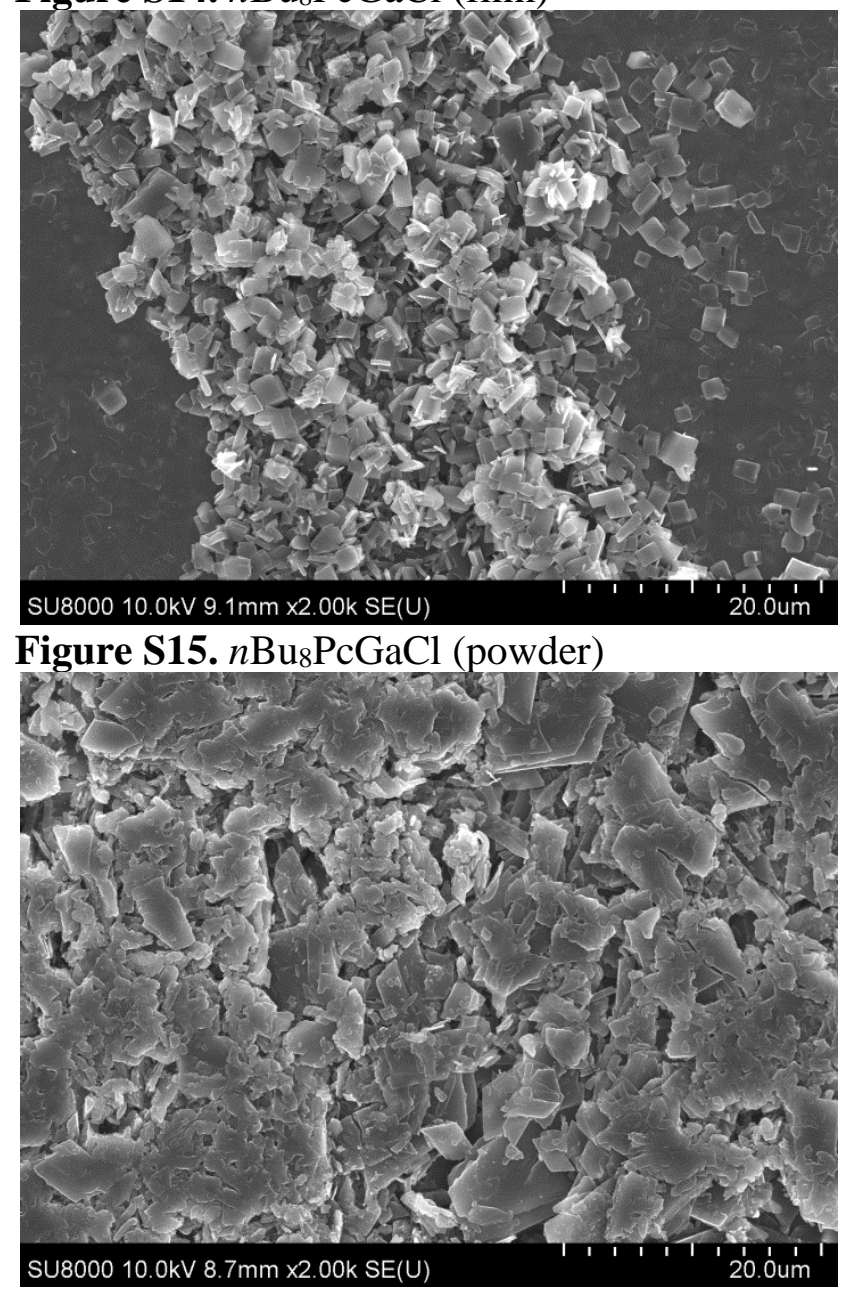

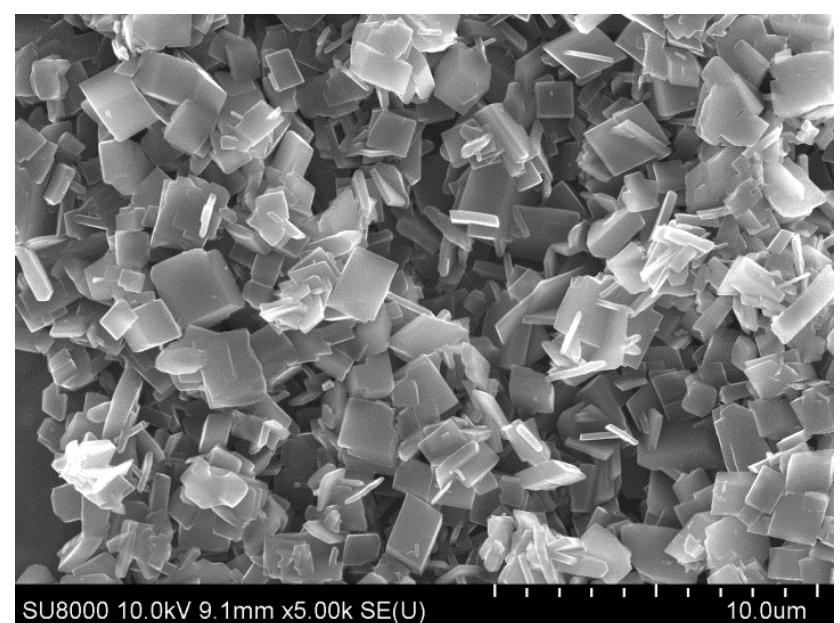

SU8000 $10.0 \mathrm{kV} 9.1 \mathrm{~mm} \times 5.00 \mathrm{k}$ SE(U)

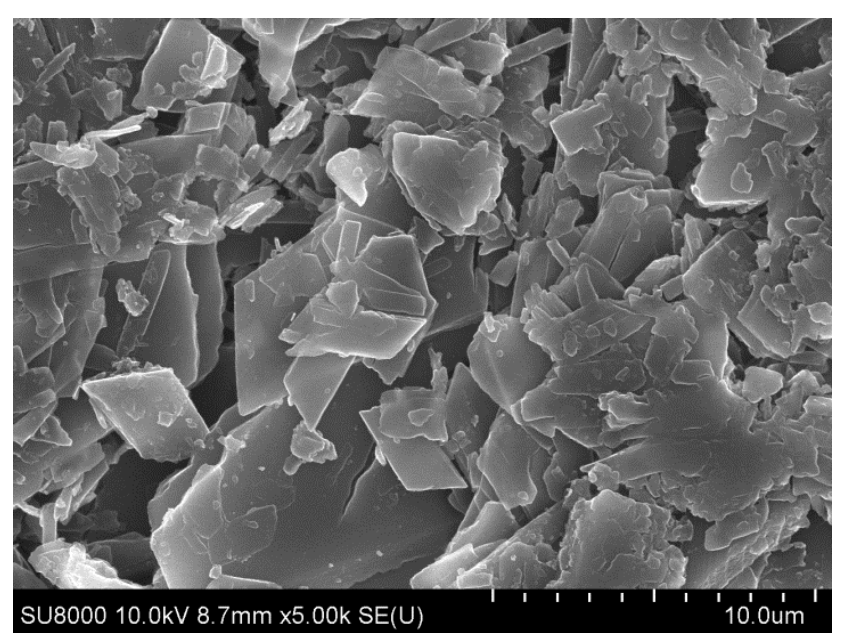

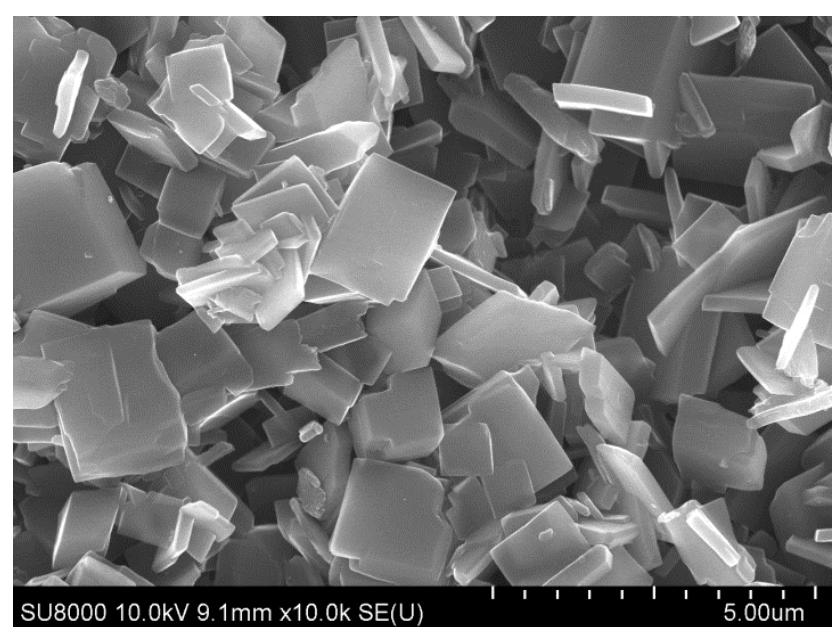

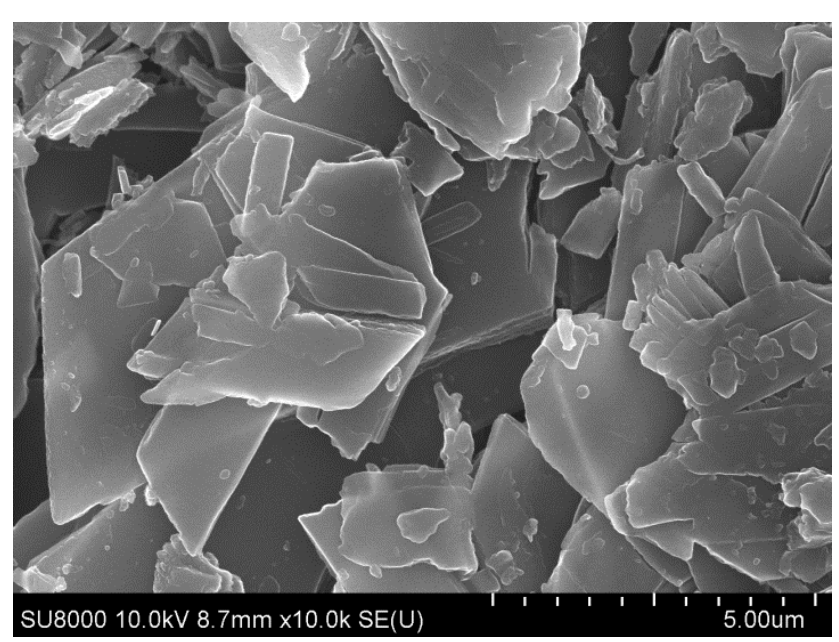




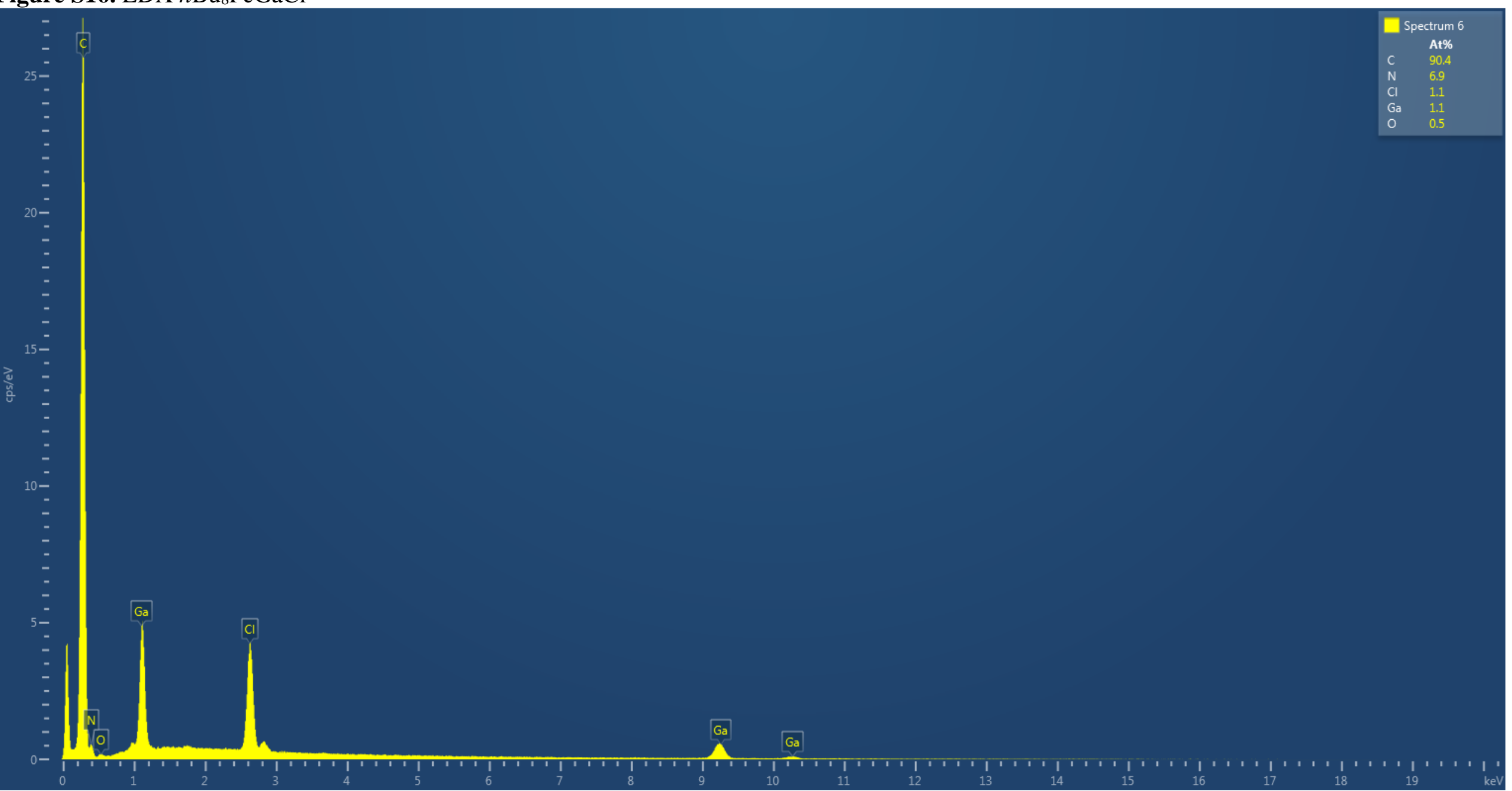


Figure S17. UV/Vis

$t \mathrm{Bu}_{4} \mathrm{PcGaCl}\left(\mathrm{CHCl}_{3}\right)$
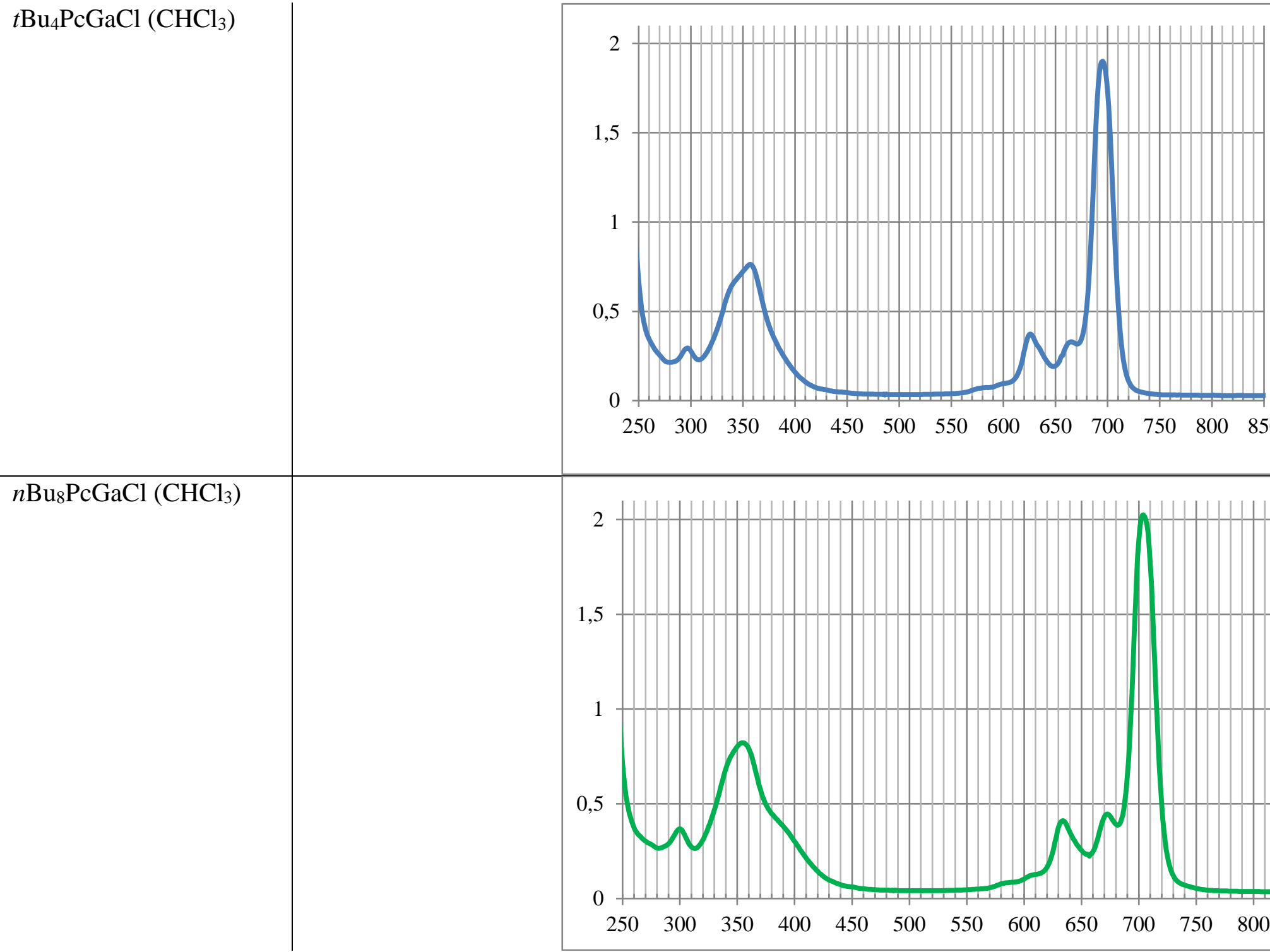

$\begin{array}{lllllllllllll}250 & 300 & 350 & 400 & 450 & 500 & 550 & 600 & 650 & 700 & 750 & 800 & 850\end{array}$

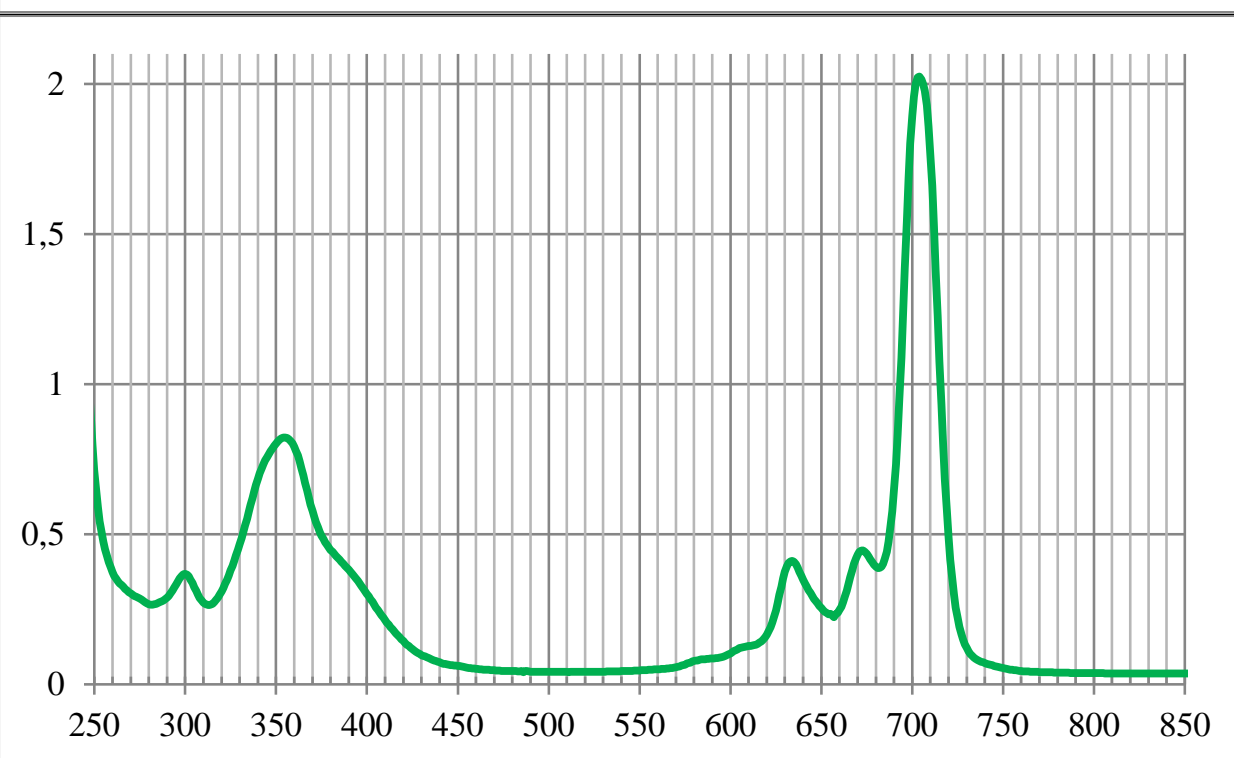


Figure S18. Crystal data for $\boldsymbol{n B u 8 P c G a C l}$

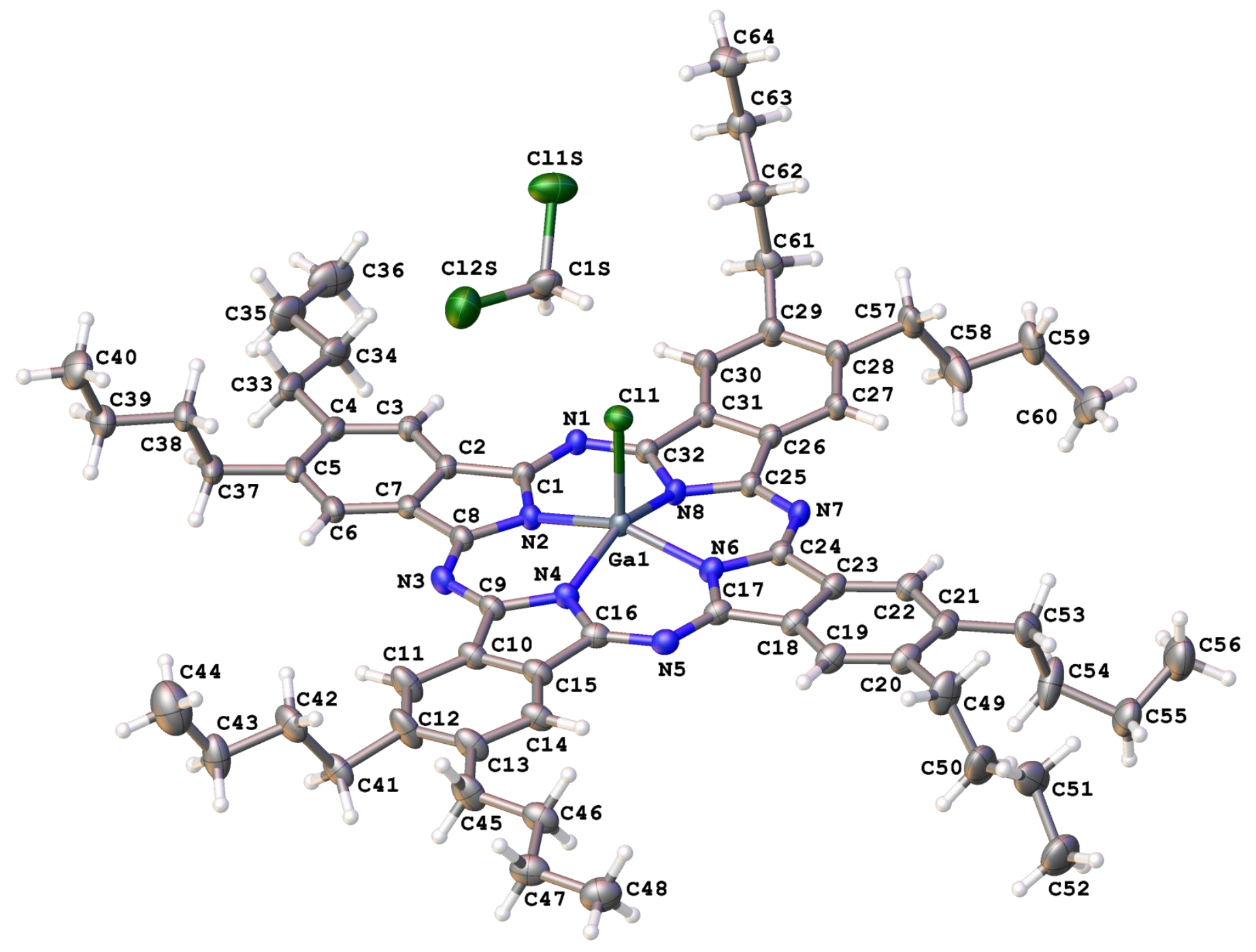


Figure S19. Crystal data for $\boldsymbol{n B u s P c G a C l}$

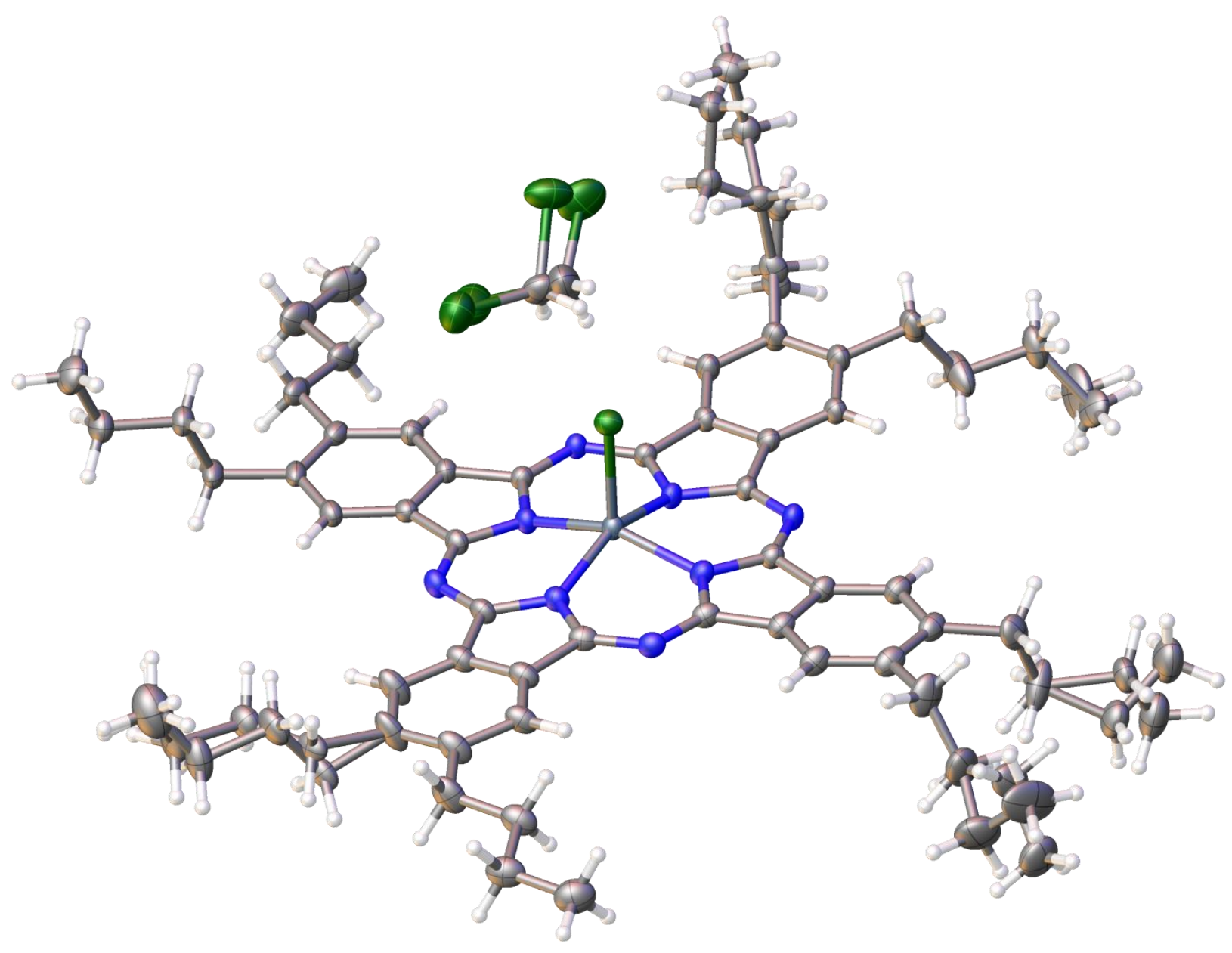


Figure S20. Crystal data for $n \mathrm{Bu} 8 \mathrm{PcGaCl}$
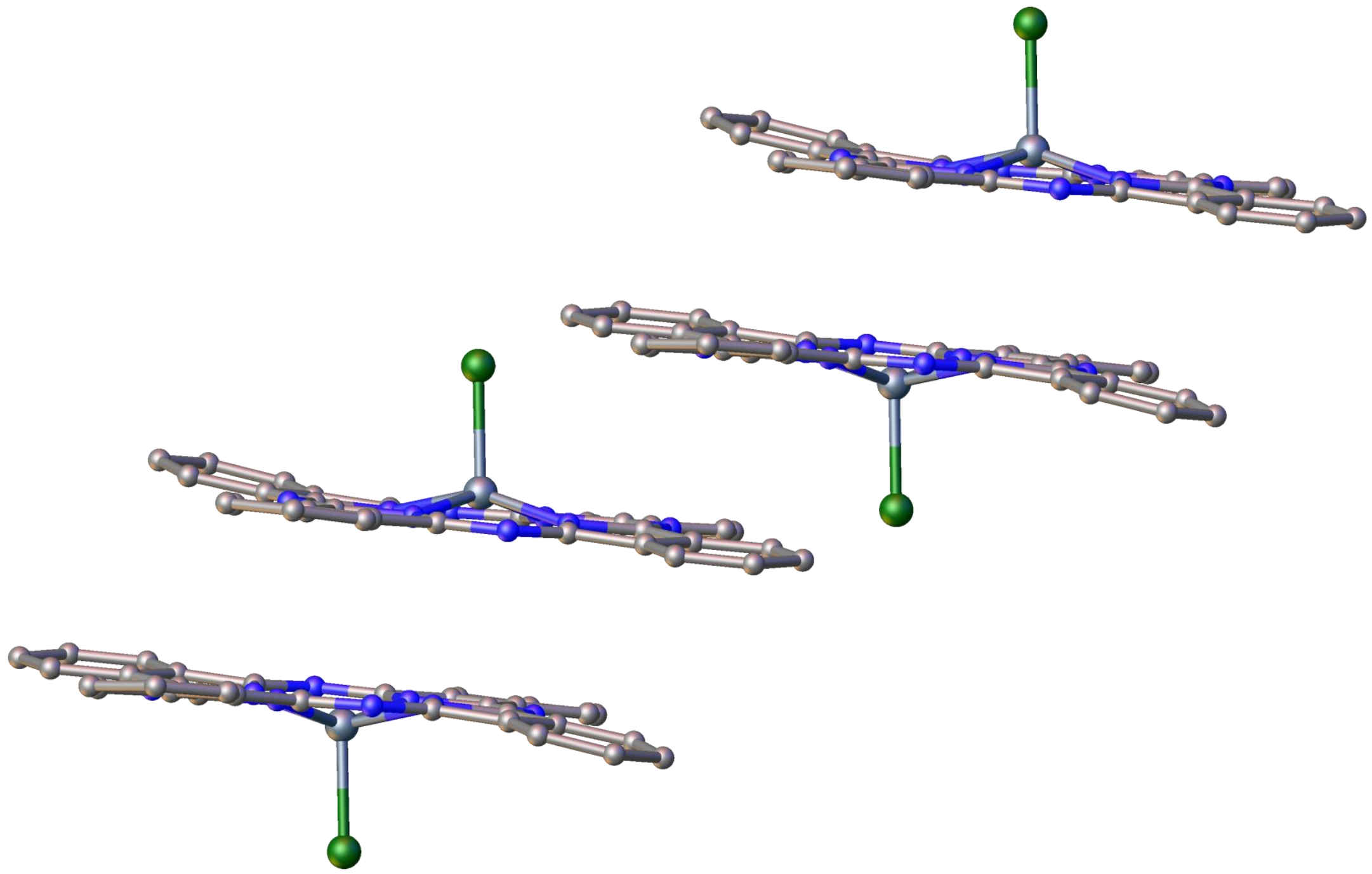

S17 


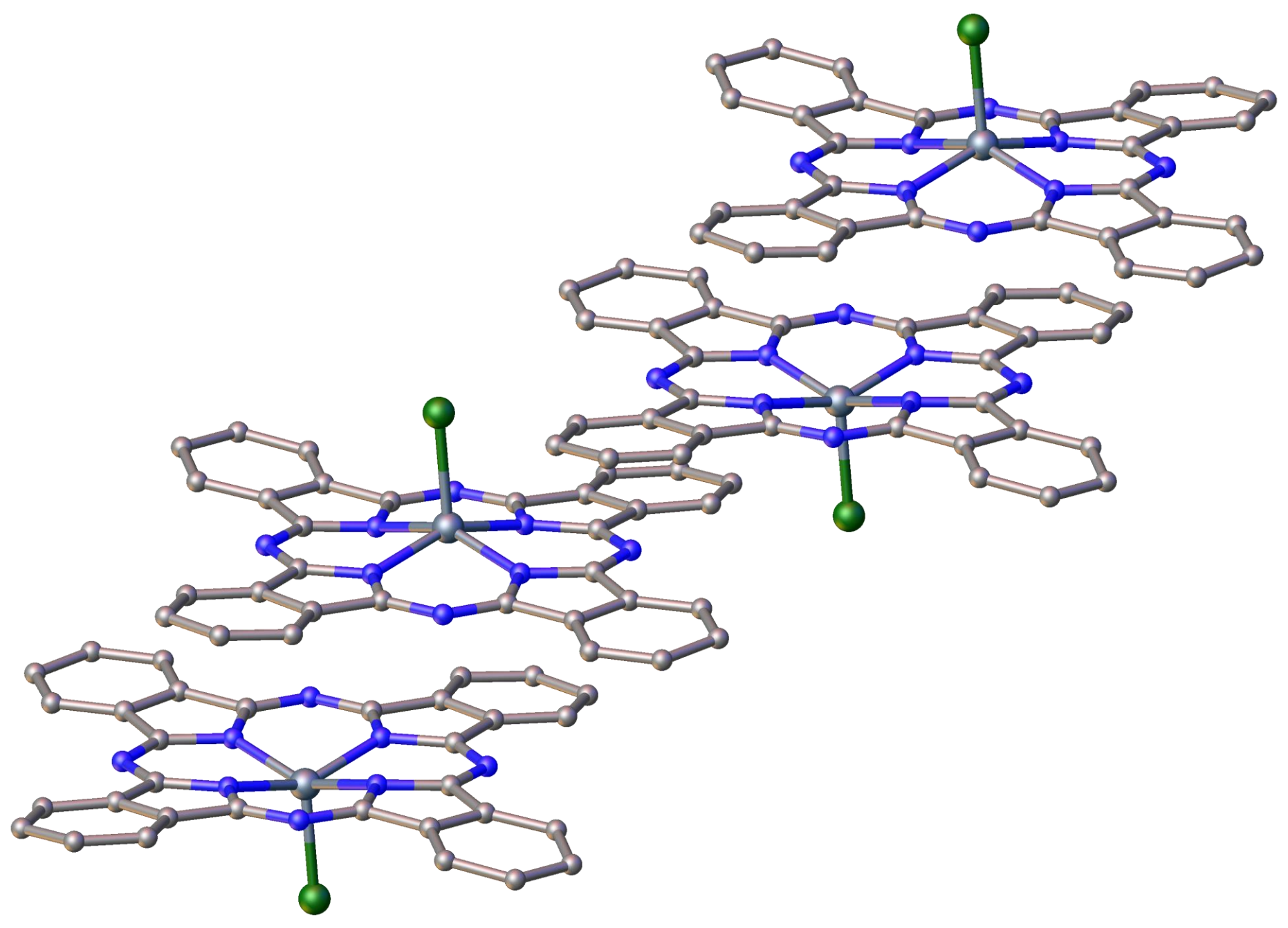




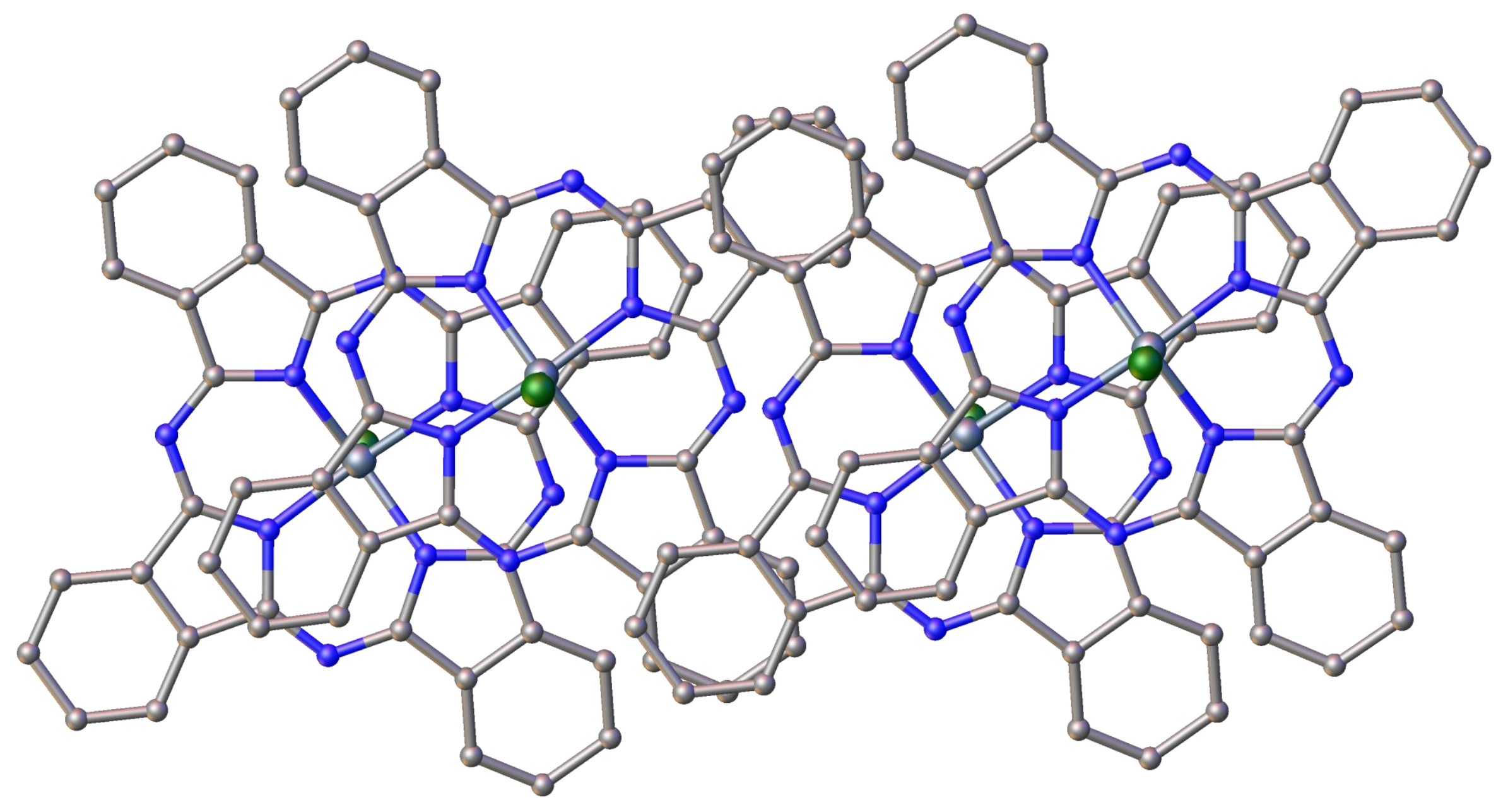


Figure S23. Crystal data for $\boldsymbol{n B u s P c G a C l}$

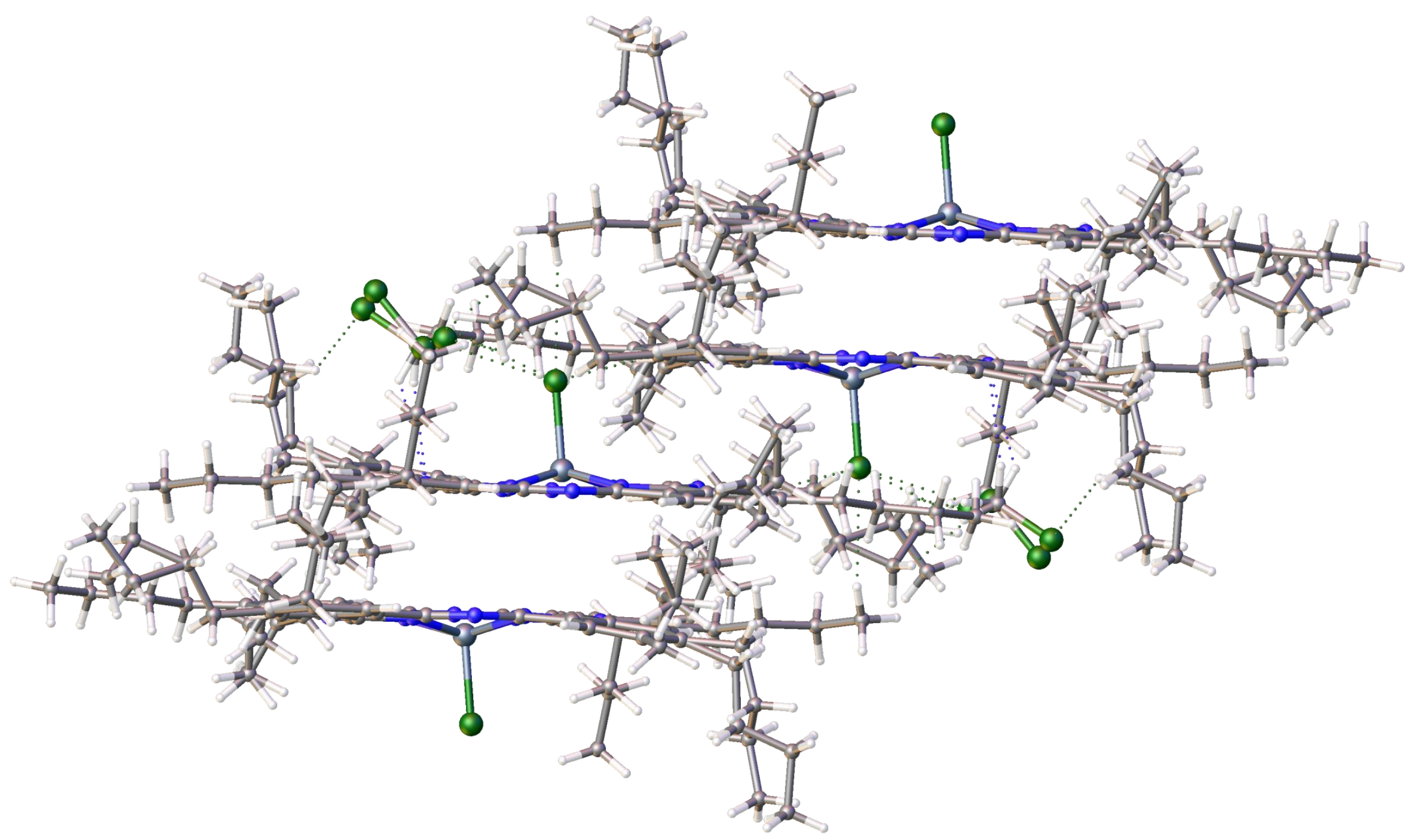


\begin{tabular}{|l|l|l|l|l|l|l|}
\hline Identification code & $n \mathrm{Bu} \mathrm{Pu}_{8} \mathrm{PcGaCl}$ \\
\hline
\end{tabular}

\begin{tabular}{|l|l|}
\hline Empirical formula & $\mathrm{C}_{65} \mathrm{H}_{82} \mathrm{Cl}_{3} \mathrm{GaN}_{8}$ \\
\hline
\end{tabular}

\begin{tabular}{|c|c|}
\hline \multicolumn{2}{|c|}{ Table S3. Crystal data and structure refinement for $n \mathrm{Bu}_{8} \mathrm{PcGaCl}$} \\
\hline Formula weight & 1151.45 \\
\hline Temperature/K & 120 \\
\hline Crystal system & triclinic \\
\hline Space group & $\mathrm{P}-1$ \\
\hline $\mathrm{a} / \AA$ & $12.2160(8)$ \\
\hline $\mathrm{b} / \AA$ & $16.4061(11)$ \\
\hline $\mathrm{c} / \AA$ & $16.6201(11)$ \\
\hline$\alpha /{ }^{\circ}$ & $66.0310(10)$ \\
\hline$\beta /{ }^{\circ}$ & $77.616(2)$ \\
\hline$\gamma /{ }^{\circ}$ & $76.7540(10)$ \\
\hline Volume $/ \AA^{3}$ & $2935.0(3)$ \\
\hline $\mathrm{Z}$ & 2 \\
\hline$\rho_{\text {calc }} \mathrm{g} / \mathrm{cm}^{3}$ & 1.303 \\
\hline$\mu / \mathrm{mm}^{-1}$ & 0.653 \\
\hline $\mathrm{F}(000)$ & 1220.0 \\
\hline Crystal size $/ \mathrm{mm}^{3}$ & $0.18 \times 0.15 \times 0.1$ \\
\hline Radiation & $\operatorname{MoK} \alpha(\lambda=0.71073)$ \\
\hline $2 \Theta$ range for data collection $/{ }^{\circ}$ & 3.05 to 61.168 \\
\hline Index ranges & $-17 \leq \mathrm{h} \leq 17,-23 \leq \mathrm{k} \leq 23,-23 \leq 1 \leq 23$ \\
\hline Reflections collected & 39693 \\
\hline Independent reflections & $17981\left[\mathrm{R}_{\text {int }}=0.0550, \mathrm{R}_{\text {sigma }}=0.0909\right]$ \\
\hline Data/restraints/parameters & $17981 / 5 / 841$ \\
\hline Goodness-of-fit on $\mathrm{F}^{2}$ & 0.980 \\
\hline Final $R$ indexes $[\mathrm{I}>=2 \sigma(\mathrm{I})]$ & $\mathrm{R}_{1}=0.0531, \mathrm{wR}_{2}=0.1152$ \\
\hline Final $\mathrm{R}$ indexes [all data] & $\mathrm{R}_{1}=0.1074, \mathrm{wR}_{2}=0.1358$ \\
\hline Largest diff. peak/hole / e $\AA^{-3}$ & $0.57 /-0.48$ \\
\hline
\end{tabular}


Table S4. Bond Lengths for $\boldsymbol{n B u}$ PcGaCl.

\begin{tabular}{|c|c|c|c|c|c|}
\hline Atom & Atom & Length/Å & Atom & Atom & Length/Å \\
\hline Ga1 & $\mathrm{Cl1}$ & $2.2197(6)$ & $\mathrm{C} 23$ & $\mathrm{C} 24$ & $1.450(3)$ \\
\hline Ga1 & $\mathrm{N} 2$ & $1.9949(18)$ & $\mathrm{C} 25$ & C26 & $1.439(3)$ \\
\hline Ga1 & $\mathrm{N} 4$ & 1.9941(18) & C26 & $\mathrm{C} 27$ & $1.395(3)$ \\
\hline Ga1 & N6 & $1.9848(18)$ & C26 & C31 & $1.397(3)$ \\
\hline Ga1 & N8 & $1.9988(17)$ & $\mathrm{C} 27$ & C28 & $1.382(3)$ \\
\hline N1 & C1 & $1.328(3)$ & C28 & C29 & $1.419(3)$ \\
\hline N1 & C32 & $1.332(3)$ & C28 & C57 & $1.523(3)$ \\
\hline $\mathrm{N} 2$ & $\mathrm{C} 1$ & $1.387(3)$ & C29 & $\mathrm{C} 30$ & $1.400(3)$ \\
\hline $\mathrm{N} 2$ & C8 & $1.382(3)$ & C29 & C61 & $1.499(15)$ \\
\hline N3 & $\mathrm{C} 8$ & $1.325(3)$ & C29 & C61A & $1.546(15)$ \\
\hline N3 & C9 & $1.329(3)$ & C30 & C31 & 1.391(3) \\
\hline N4 & C9 & $1.380(3)$ & C31 & C32 & $1.452(3)$ \\
\hline N4 & $\mathrm{C} 16$ & $1.382(3)$ & C33 & C34 & $1.515(4)$ \\
\hline N5 & C16 & $1.323(3)$ & C34 & $\mathrm{C} 35$ & $1.537(3)$ \\
\hline N5 & $\mathrm{C} 17$ & $1.328(3)$ & C35 & C36 & $1.516(4)$ \\
\hline N6 & C17 & $1.379(3)$ & C37 & C38 & $1.530(3)$ \\
\hline N6 & $\mathrm{C} 24$ & $1.381(3)$ & C38 & C39 & $1.524(3)$ \\
\hline N7 & $\mathrm{C} 24$ & $1.324(3)$ & C39 & C40 & $1.509(4)$ \\
\hline N7 & $\mathrm{C} 25$ & $1.328(3)$ & $\mathrm{C} 44$ & C43 & $1.457(6)$ \\
\hline $\mathrm{N} 8$ & $\mathrm{C} 25$ & $1.384(3)$ & $\mathrm{C} 44$ & $\mathrm{C} 43 \mathrm{~A}$ & $1.511(7)$ \\
\hline $\mathrm{N} 8$ & C32 & $1.373(3)$ & $\mathrm{C} 45$ & C46 & $1.510(4)$ \\
\hline $\mathrm{C} 1$ & $\mathrm{C} 2$ & $1.455(3)$ & $\mathrm{C} 46$ & C47 & $1.522(3)$ \\
\hline
\end{tabular}

Table S5. Bond Angles for $\boldsymbol{n B u} 8$ PcGaCl.

\begin{tabular}{|c|c|c|c|c|c|c|c|}
\hline Atom & Atom & Atom & Angle $/^{\circ}$ & Atom & Atom & Atom & Angle $/^{\circ}$ \\
\hline $\mathrm{N} 2$ & $\mathrm{Ga} 1$ & $\mathrm{Cl1}$ & 101.97(6) & $\mathrm{C} 20$ & C19 & C18 & $119.2(2)$ \\
\hline $\mathrm{N} 2$ & Ga1 & N8 & $86.78(7)$ & C19 & $\mathrm{C} 20$ & $\mathrm{C} 21$ & $120.2(2)$ \\
\hline $\mathrm{N} 4$ & $\mathrm{Ga} 1$ & $\mathrm{Cl1}$ & $107.27(6)$ & C19 & $\mathrm{C} 20$ & C49 & $118.8(2)$ \\
\hline N4 & Ga1 & $\mathrm{N} 2$ & $87.32(7)$ & $\mathrm{C} 21$ & $\mathrm{C} 20$ & C49 & $120.9(2)$ \\
\hline N4 & $\mathrm{Ga} 1$ & $\mathrm{~N} 8$ & $154.32(8)$ & $\mathrm{C} 20$ & $\mathrm{C} 21$ & C53 & $122.7(2)$ \\
\hline N6 & Ga1 & Cl1 & $102.76(6)$ & $\mathrm{C} 22$ & $\mathrm{C} 21$ & C20 & $119.9(2)$ \\
\hline N6 & Ga1 & $\mathrm{N} 2$ & $155.19(8)$ & $\mathrm{C} 22$ & C21 & C53 & $117.3(2)$ \\
\hline N6 & Ga1 & N4 & 87.25 & $\mathrm{C} 23$ & $\mathrm{C} 22$ & C21 & $119.4(2)$ \\
\hline N6 & Ga1 & $\mathrm{N} 8$ & $87.71(7)$ & C18 & C23 & $\mathrm{C} 24$ & 106.61(19 \\
\hline N8 & Ga1 & Cl1 & 98.40 & C22 & C23 & C18 & $120.8(2)$ \\
\hline $\mathrm{C} 1$ & N1 & $\mathrm{C} 32$ & $122.42(18)$ & $\mathrm{C} 22$ & $\mathrm{C} 23$ & $\mathrm{C} 24$ & $132.6(2)$ \\
\hline $\mathrm{C} 1$ & $\mathrm{~N} 2$ & Ga1 & $125.13(14)$ & N6 & $\mathrm{C} 24$ & $\mathrm{C} 23$ & $109.50(18$ \\
\hline C8 & $\mathrm{N} 2$ & Ga1 & $125.28(14)$ & N7 & $\mathrm{C} 24$ & N6 & $127.47(19$ \\
\hline $\mathrm{C} 8$ & $\mathrm{~N} 2$ & C1 & 107.74(17) & $\mathrm{N} 7$ & $\mathrm{C} 24$ & C23 & $123.0(2)$ \\
\hline $\mathrm{C} 8$ & N3 & $\mathrm{C} 9$ & $122.10(19)$ & $\mathrm{N} 7$ & $\mathrm{C} 25$ & N8 & $127.54(19$ \\
\hline C9 & N4 & Ga1 & $126.36(14)$ & N7 & $\mathrm{C} 25$ & $\mathrm{C} 26$ & $122.95(19$ \\
\hline C9 & N4 & $\mathrm{C} 16$ & $107.57(17)$ & $\mathrm{N} 8$ & $\mathrm{C} 25$ & $\mathrm{C} 26$ & $109.46(18$ \\
\hline $\mathrm{C} 16$ & N4 & Ga1 & $125.52(15)$ & $\mathrm{C} 27$ & $\mathrm{C} 26$ & $\mathrm{C} 25$ & 132.2(2) \\
\hline $\mathrm{C} 16$ & N5 & $\mathrm{C} 17$ & $121.84(19)$ & $\mathrm{C} 27$ & $\mathrm{C} 26$ & C31 & $120.6(2)$ \\
\hline $\mathrm{C} 17$ & N6 & Ga1 & $125.63(14)$ & C31 & $\mathrm{C} 26$ & $\mathrm{C} 25$ & $106.95(18$ \\
\hline $\mathrm{C} 17$ & N6 & $\mathrm{C} 24$ & $107.67(17)$ & $\mathrm{C} 28$ & $\mathrm{C} 27$ & $\mathrm{C} 26$ & 119.3(2) \\
\hline $\mathrm{C} 24$ & N6 & Ga1 & $125.54(14)$ & C27 & $\mathrm{C} 28$ & C29 & $120.4(2)$ \\
\hline
\end{tabular}




\begin{tabular}{|l|l|l|l|l|l||}
\hline C2 & C3 & $1.393(3)$ & C47 & C48 & $1.512(4)$ \\
\hline C2 & C7 & $1.390(3)$ & C49 & C50 & $1.493(4)$ \\
\hline C3 & C4 & $1.401(3)$ & C50 & C51 & $1.600(5)$ \\
\hline C4 & C5 & $1.423(3)$ & C50 & C51A & $1.481(9)$ \\
\hline \hline C4 & C33 & $1.514(3)$ & C53 & C54 & $1.518(4)$ \\
\hline C5 & C6 & $1.390(3)$ & C54 & C55 & $1.532(6)$ \\
\hline C5 & C37 & $1.520(3)$ & C54 & C55A & $1.603(7)$ \\
\hline C6 & C7 & $1.390(3)$ & C57 & C58 & $1.485(4)$ \\
\hline C7 & C8 & $1.449(3)$ & C58 & C59 & $1.510(4)$ \\
\hline \hline C9 & C10 & $1.449(3)$ & C59 & C60 & $1.590(11)$ \\
\hline C10 & C11 & $1.391(3)$ & C59 & C60A & $1.513(6)$ \\
\hline C10 & C15 & $1.388(3)$ & C41 & C42 & $1.515(8)$ \\
\hline C11 & C12 & $1.384(3)$ & C42 & C43 & $1.513(7)$ \\
\hline C12 & C13 & $1.422(4)$ & C51 & C52 & $1.496(6)$ \\
\hline C12 & C41 & $1.497(6)$ & C55 & C56 & $1.518(9)$ \\
\hline C12 & C41A & $1.622(8)$ & C61 & C62 & $1.545(11)$ \\
\hline C13 & C14 & $1.393(3)$ & C62 & C63 & $1.496(7)$ \\
\hline C13 & C45 & $1.515(3)$ & C63 & C64 & $1.523(7)$ \\
\hline C14 & C15 & $1.396(3)$ & C41A & C42A & $1.502(10)$ \\
\hline \hline C15 & C16 & $1.453(3)$ & C42A & C43A & $1.558(8)$ \\
\hline C17 & C18 & $1.452(3)$ & C51A & C52A & $1.479(13)$ \\
\hline C18 & C19 & $1.397(3)$ & C55A & C56A & $1.511(10)$ \\
\hline C18 & C23 & $1.390(3)$ & C61A & C62A & $1.516(13)$ \\
\hline C19 & C20 & $1.382(3)$ & C62A & C63A & $1.526(7)$ \\
\hline C20 & C21 & $1.420(3)$ & C63A & C64A & $1.510(8)$ \\
\hline C20 & C49 & $1.518(3)$ & C13S & C2S & $1.704(17)$ \\
\hline & & & & \\
\hline
\end{tabular}

\begin{tabular}{|c|c|c|c|c|c|c|c|}
\hline $\mathrm{C} 24$ & N7 & $\mathrm{C} 25$ & $122.85(19)$ & $\mathrm{C} 27$ & $\mathrm{C} 28$ & C57 & $117.5(2)$ \\
\hline $\mathrm{C} 25$ & N8 & Ga1 & $124.22(14)$ & C29 & $\mathrm{C} 28$ & C57 & 122.1(2) \\
\hline C32 & N8 & Ga1 & $124.36(14)$ & $\mathrm{C} 28$ & $\mathrm{C} 29$ & C61 & $120.8(6)$ \\
\hline C32 & N8 & C25 & $107.71(17)$ & $\mathrm{C} 28$ & $\mathrm{C} 29$ & C61A & $122.6(6)$ \\
\hline $\mathrm{N} 1$ & $\mathrm{C} 1$ & $\mathrm{~N} 2$ & $127.03(18)$ & C30 & C29 & C28 & $119.9(2)$ \\
\hline $\mathrm{N} 1$ & $\mathrm{C} 1$ & $\mathrm{C} 2$ & $123.69(19)$ & C30 & C29 & C61 & $117.5(6)$ \\
\hline $\mathrm{N} 2$ & $\mathrm{C} 1$ & $\mathrm{C} 2$ & $109.27(17)$ & C30 & C29 & C61A & $117.3(6)$ \\
\hline C3 & $\mathrm{C} 2$ & C1 & $132.8(2)$ & C31 & C30 & $\mathrm{C} 29$ & 119.1(2) \\
\hline C7 & $\mathrm{C} 2$ & C1 & $106.44(18)$ & C26 & C31 & C32 & $106.38(18)$ \\
\hline C7 & $\mathrm{C} 2$ & C3 & $120.70(19)$ & C30 & C31 & $\mathrm{C} 26$ & $120.67(19)$ \\
\hline $\mathrm{C} 2$ & C3 & C4 & $119.5(2)$ & C30 & C31 & C32 & $132.7(2)$ \\
\hline C3 & $\mathrm{C} 4$ & C5 & 119.1(2) & N1 & C32 & N8 & $127.67(19$ \\
\hline C3 & $\mathrm{C} 4$ & C33 & 121.0(2) & N1 & C32 & C31 & $122.82(19)$ \\
\hline C5 & $\mathrm{C} 4$ & C33 & 119.9(2) & N8 & C32 & C31 & $109.49(18$ \\
\hline $\mathrm{C} 4$ & $\mathrm{C} 5$ & C37 & $122.5(2)$ & C4 & C33 & C34 & 117.1(2) \\
\hline C6 & C5 & C4 & $120.6(2)$ & C33 & C34 & C35 & 113.3(2) \\
\hline C6 & C5 & C37 & 116.9(2) & C36 & C35 & C34 & $113.9(2)$ \\
\hline C5 & C6 & C7 & $119.3(2)$ & C5 & C37 & C38 & $112.18(19$ \\
\hline $\mathrm{C} 2$ & C7 & C6 & $120.8(2)$ & C39 & C38 & C37 & $113.5(2)$ \\
\hline $\mathrm{C} 2$ & C7 & C8 & $107.47(18)$ & $\mathrm{C} 40$ & C39 & C38 & $112.8(2)$ \\
\hline C6 & C7 & C8 & $131.7(2)$ & C46 & C45 & C13 & $117.5(2)$ \\
\hline $\mathrm{N} 2$ & C8 & C7 & $109.07(18)$ & C45 & C46 & C47 & $112.0(2)$ \\
\hline $\mathrm{N} 3$ & C8 & $\mathrm{N} 2$ & $128.2(2)$ & C48 & C47 & C46 & 112.9(2) \\
\hline $\mathrm{N} 3$ & C8 & C7 & $122.73(19)$ & C50 & C49 & C20 & $116.9(3)$ \\
\hline $\mathrm{N} 3$ & C9 & N4 & $127.58(19)$ & $\mathrm{C} 49$ & C50 & C51 & $106.1(3)$ \\
\hline $\mathrm{N} 3$ & C9 & $\mathrm{C} 10$ & $122.9(2)$ & C51A & C50 & C49 & $138.3(6)$ \\
\hline
\end{tabular}




\begin{tabular}{|c|c|c|c|c|c|}
\hline C21 & $\mathrm{C} 22$ & $1.388(3)$ & $\overline{\mathrm{Cl} 4 \mathrm{~S}}$ & $\mathrm{C} 2 \mathrm{~S}$ & $1.758(13)$ \\
\hline C21 & C53 & $1.516(3)$ & $\mathrm{Cl1S}$ & C1S & $1.778(11)$ \\
\hline $\mathrm{C} 22$ & $\mathrm{C} 23$ & $1.384(3)$ & $\mathrm{Cl} 2 \mathrm{~S}$ & C1S & $1.737(9)$ \\
\hline
\end{tabular}

\begin{tabular}{|c|c|c|c|c|c|c|c|}
\hline N4 & C9 & C10 & $109.47(19)$ & $\mathrm{C} 21$ & C53 & C54 & $111.5(2)$ \\
\hline $\mathrm{C} 11$ & $\mathrm{C} 10$ & $\mathrm{C} 9$ & 132.2(2) & C53 & C54 & C55 & $120.6(3)$ \\
\hline $\mathrm{C} 15$ & $\mathrm{C} 10$ & C9 & $106.89(19)$ & C53 & C54 & C55A & $102.9(4)$ \\
\hline $\mathrm{C} 15$ & $\mathrm{C} 10$ & $\mathrm{C} 11$ & $121.0(2)$ & C58 & C57 & C28 & $113.8(2)$ \\
\hline $\mathrm{C} 12$ & C11 & $\mathrm{C} 10$ & $118.5(2)$ & C57 & C58 & C59 & 116.1(3) \\
\hline $\mathrm{C} 11$ & $\mathrm{C} 12$ & $\mathrm{C} 13$ & $121.0(2)$ & C58 & C59 & C60 & $115.5(6)$ \\
\hline $\mathrm{C} 11$ & $\mathrm{C} 12$ & C41 & 118.4(3) & C58 & C59 & C60A & $114.1(3)$ \\
\hline $\mathrm{C} 11$ & $\mathrm{C} 12$ & C41A & $118.1(4)$ & $\mathrm{C} 12$ & C41 & C42 & $107.8(5)$ \\
\hline $\mathrm{C} 13$ & $\mathrm{C} 12$ & $\mathrm{C} 41$ & $119.6(3)$ & C43 & C42 & C41 & $110.9(5)$ \\
\hline $\mathrm{C} 13$ & $\mathrm{C} 12$ & C41A & $118.9(3)$ & $\mathrm{C} 44$ & C43 & C42 & $109.4(5)$ \\
\hline $\mathrm{C} 12$ & C13 & $\mathrm{C} 45$ & $119.7(2)$ & C52 & C51 & C50 & $111.0(4)$ \\
\hline C14 & $\mathrm{C} 13$ & $\mathrm{C} 12$ & 119.7(2) & C56 & $\mathrm{C} 55$ & C54 & $106.6(5)$ \\
\hline $\mathrm{C} 14$ & C13 & $\mathrm{C} 45$ & $120.6(2)$ & $\mathrm{C} 29$ & C61 & C62 & $114.4(6)$ \\
\hline $\mathrm{C} 13$ & $\mathrm{C} 14$ & $\mathrm{C} 15$ & 118.6(2) & $\mathrm{C63}$ & C62 & C61 & $116.6(6)$ \\
\hline $\mathrm{C} 10$ & C15 & $\mathrm{C} 14$ & 121.2(2) & C62 & C63 & C64 & $110.5(4)$ \\
\hline $\mathrm{C} 10$ & $\mathrm{C} 15$ & $\mathrm{C} 16$ & $106.77(19)$ & $\mathrm{C} 42 \mathrm{~A}$ & $\mathrm{C} 41 \mathrm{~A}$ & C12 & $118.9(5)$ \\
\hline $\mathrm{C} 14$ & C15 & $\mathrm{C} 16$ & 132.1(2) & C41A & $\mathrm{C} 42 \mathrm{~A}$ & C43A & $112.6(6)$ \\
\hline $\mathrm{N} 4$ & $\mathrm{C} 16$ & $\mathrm{C} 15$ & $109.28(19)$ & C44 & $\mathrm{C} 43 \mathrm{~A}$ & C42A & $122.5(6)$ \\
\hline N5 & $\mathrm{C} 16$ & $\mathrm{~N} 4$ & $128.0(2)$ & C52A & C51A & C50 & $99.3(9)$ \\
\hline N5 & $\mathrm{C} 16$ & $\mathrm{C} 15$ & $122.7(2)$ & C56A & C55A & C54 & $106.9(6)$ \\
\hline N5 & $\mathrm{C} 17$ & N6 & $128.0(2)$ & C62A & C61A & C29 & $108.8(6)$ \\
\hline N5 & C17 & $\mathrm{C} 18$ & $122.80(19)$ & C61A & C62A & C63A & $114.1(7)$ \\
\hline N6 & $\mathrm{C} 17$ & $\mathrm{C} 18$ & $109.23(18)$ & C64A & C63A & C62A & $113.9(4)$ \\
\hline C19 & C18 & $\mathrm{C} 17$ & $132.6(2)$ & $\mathrm{Cl} 3 \mathrm{~S}$ & $\mathrm{C} 2 \mathrm{~S}$ & $\mathrm{Cl} 4 \mathrm{~S}$ & $115.2(10)$ \\
\hline $\mathrm{C} 23$ & $\mathrm{C} 18$ & $\mathrm{C} 17$ & 106.99(18) & $\mathrm{Cl} 2 \mathrm{~S}$ & $\mathrm{C} 1 \mathrm{~S}$ & Cl1S & $111.8(6)$ \\
\hline $\mathrm{C} 23$ & C18 & C19 & $120.4(2)$ & & & & \\
\hline
\end{tabular}




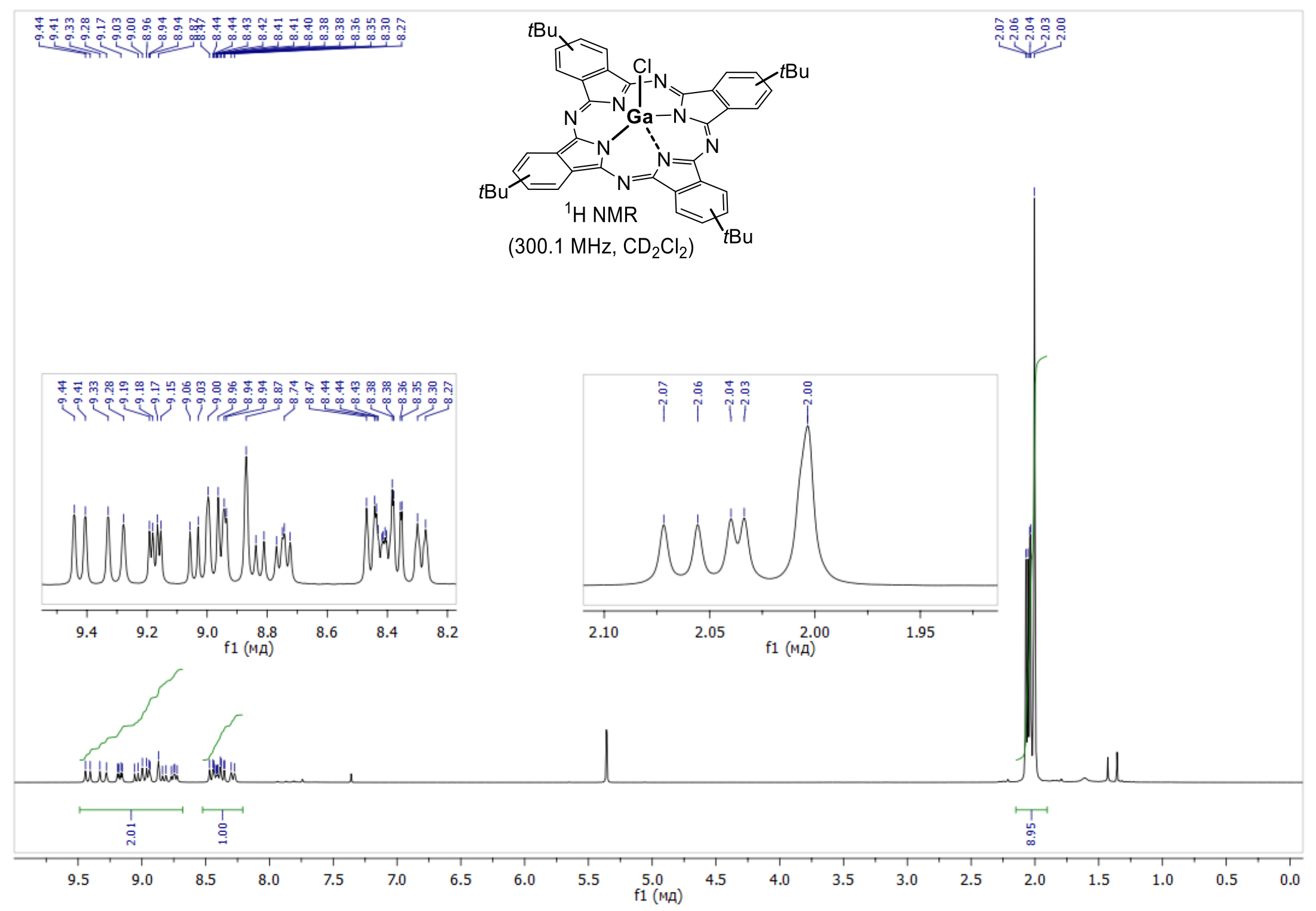


Figure S25.

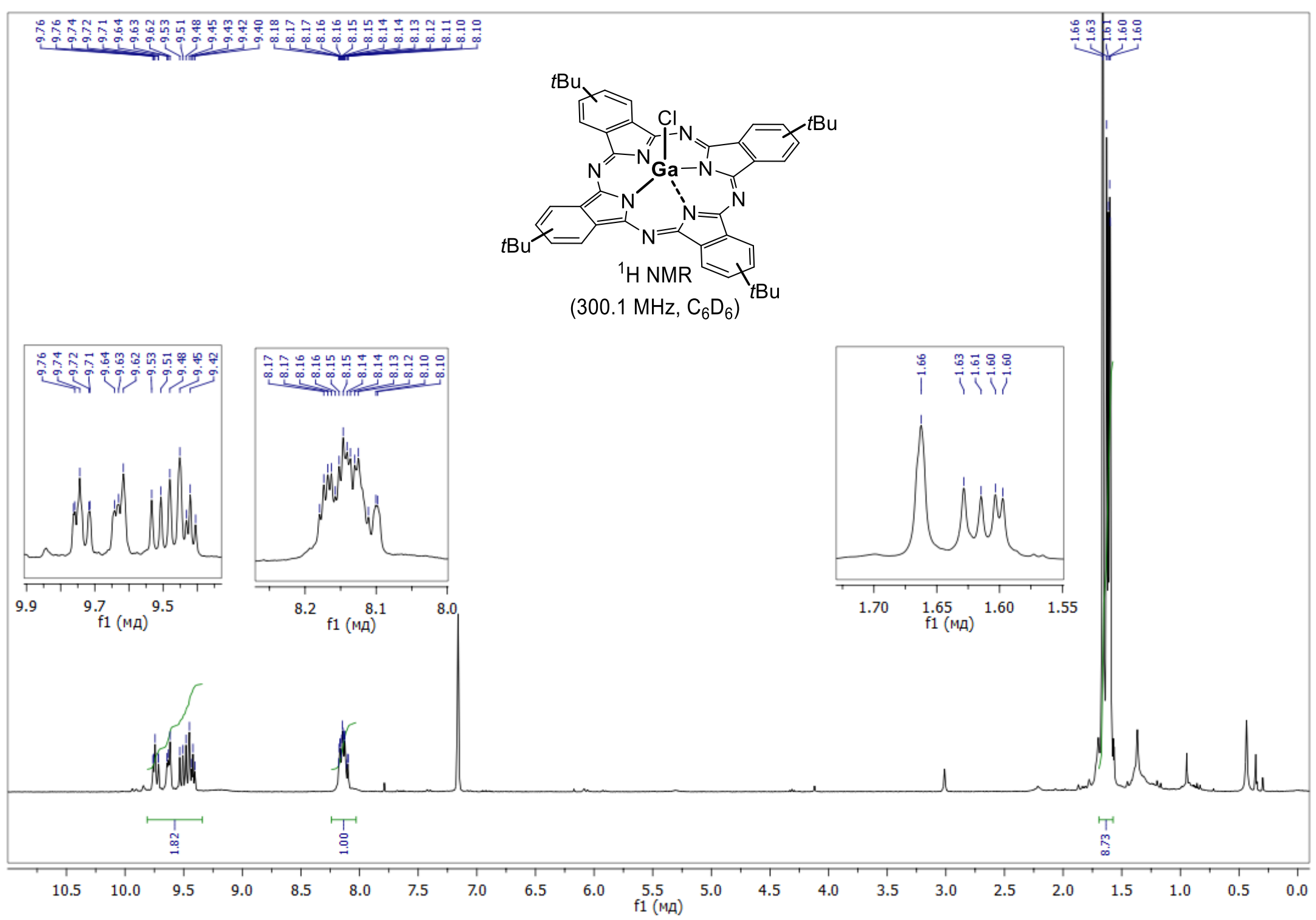


Figure S26.

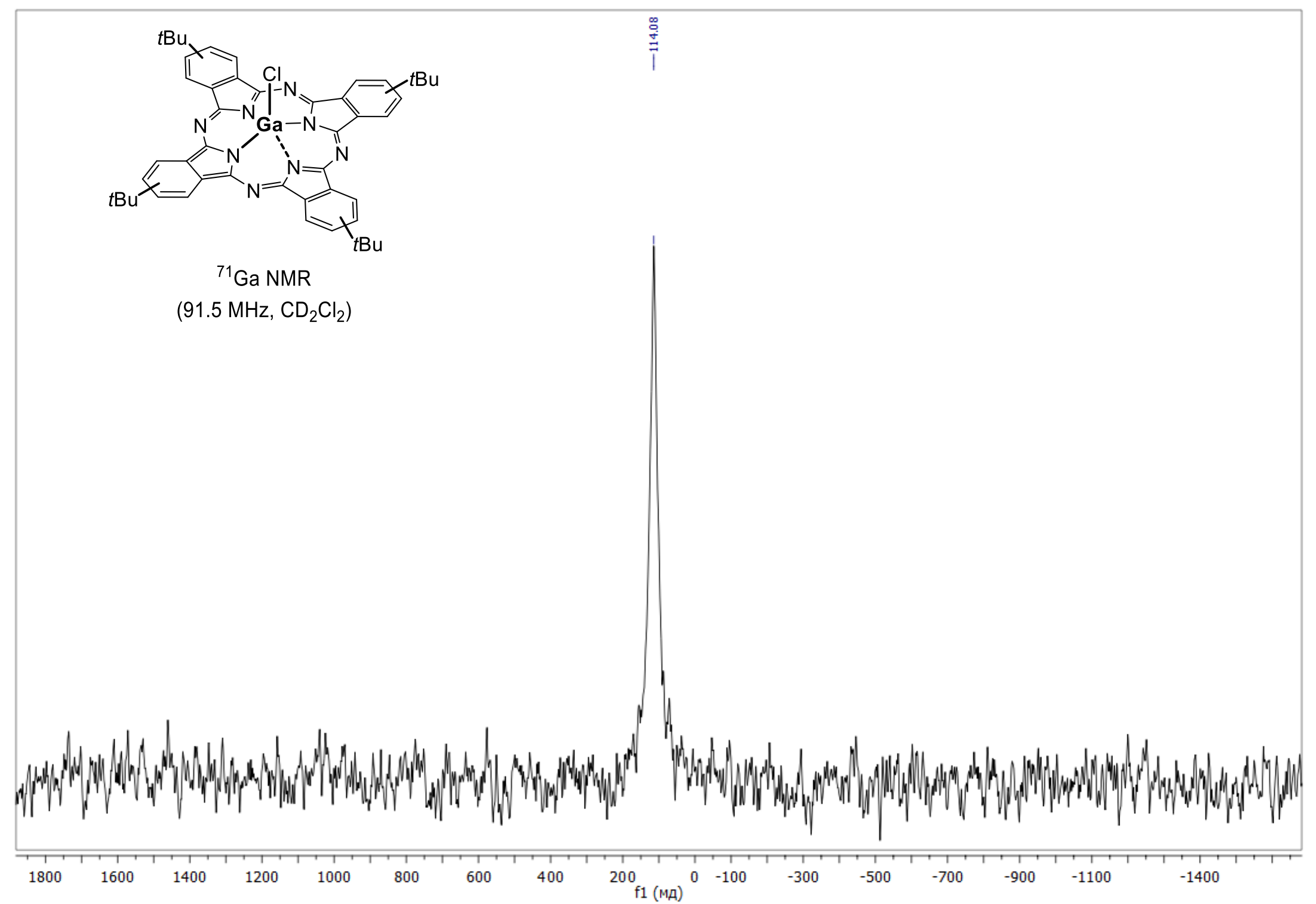


Figure S27.

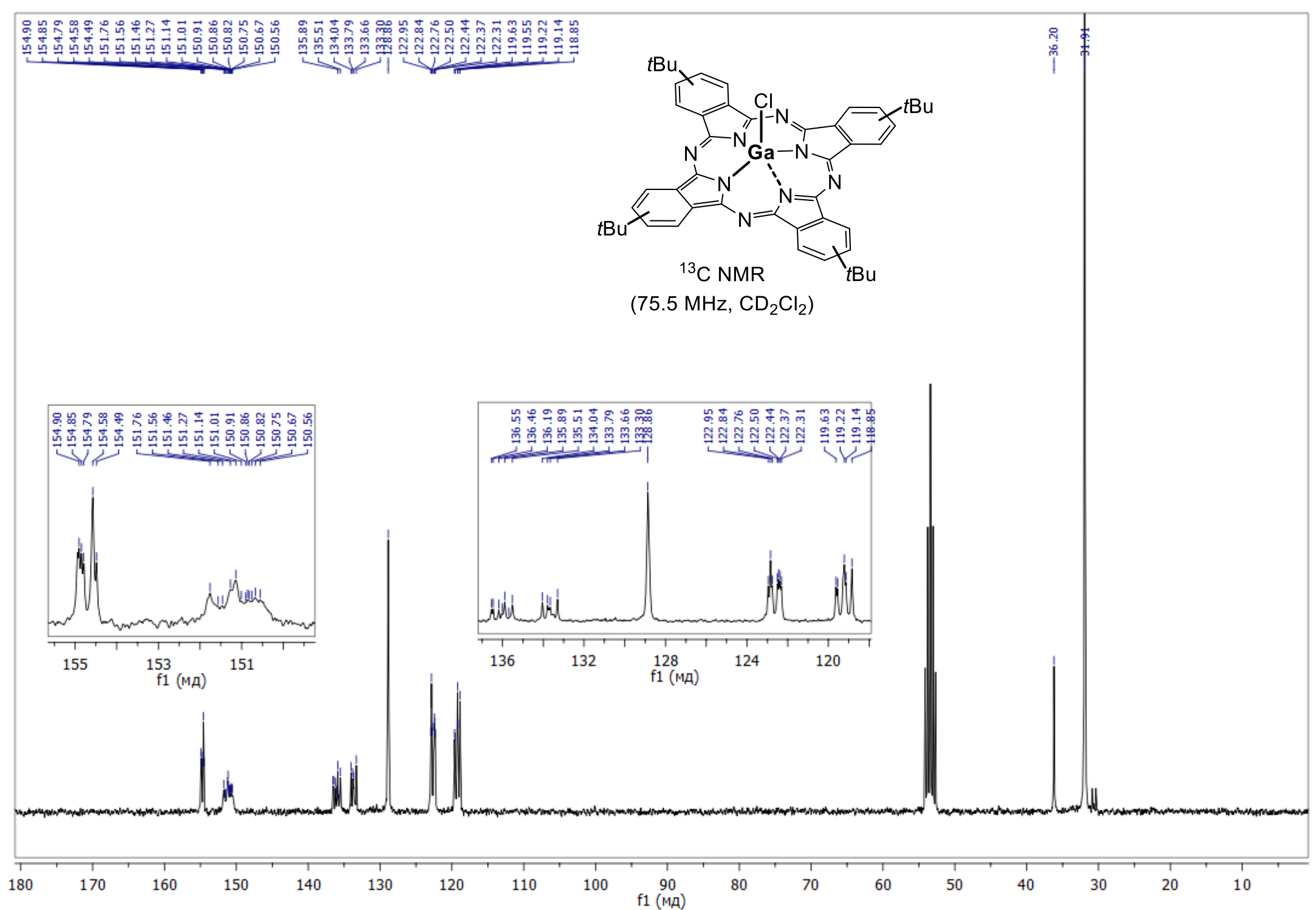


Figure S28.
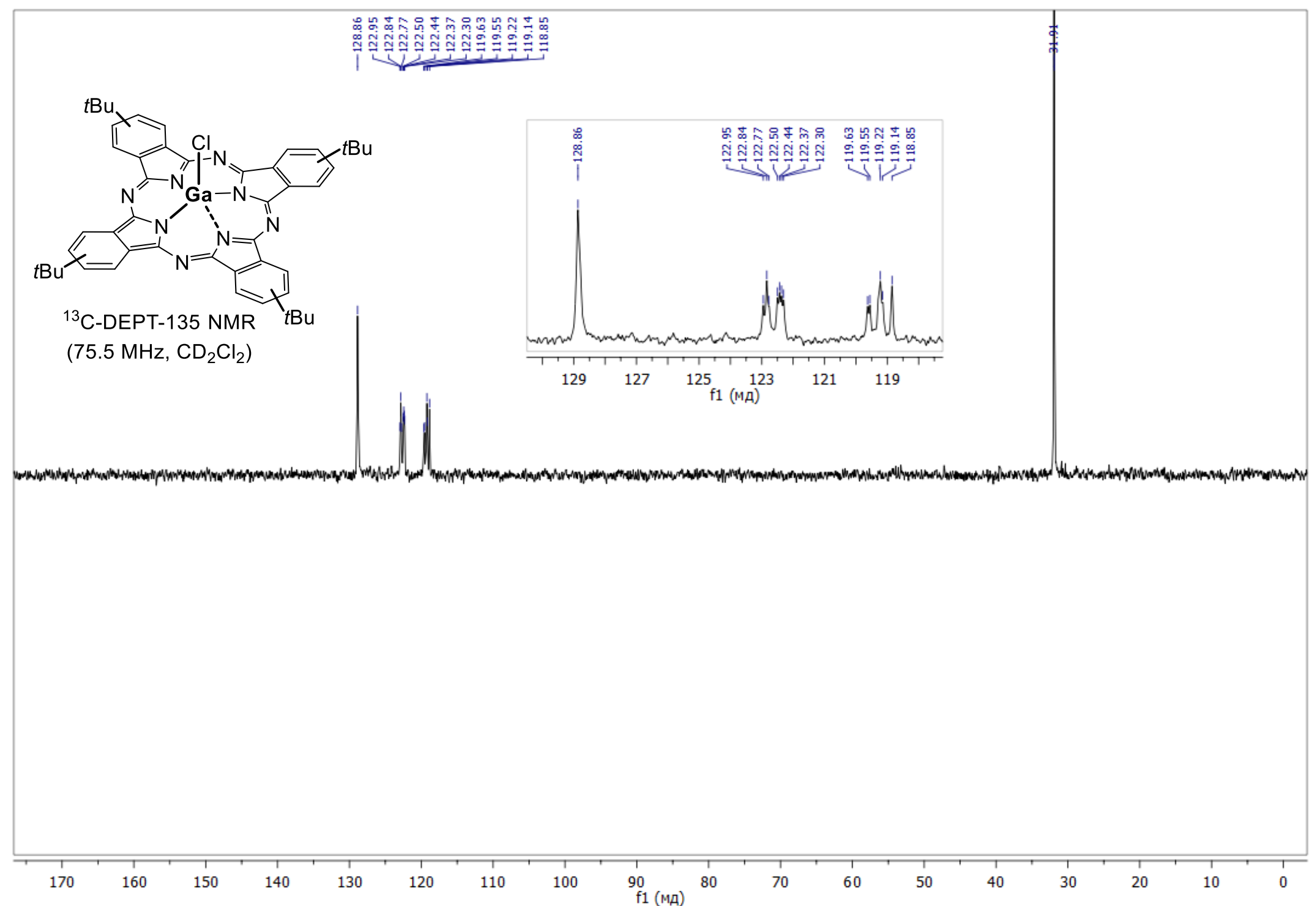
Figure S29.

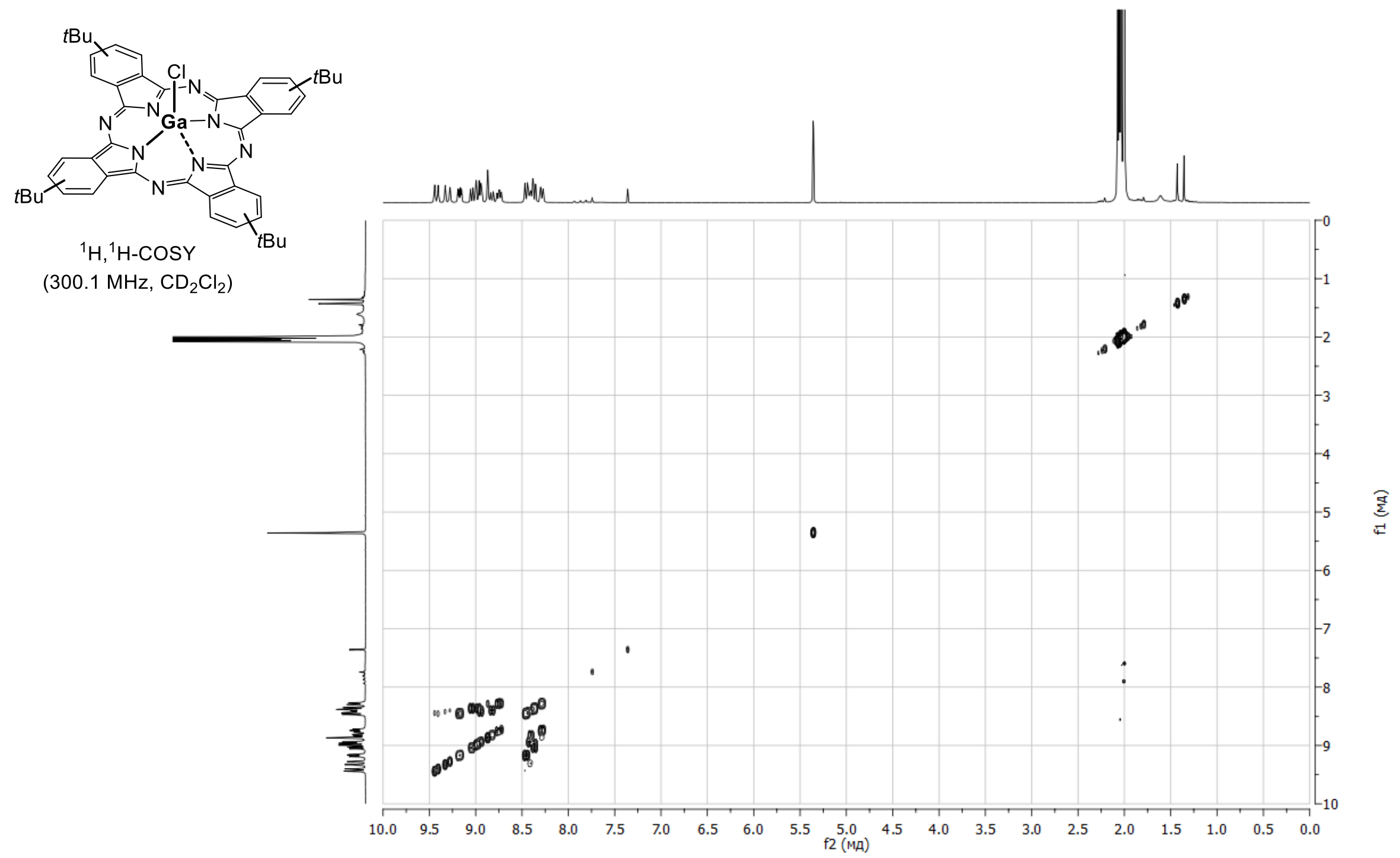


Figure S30.

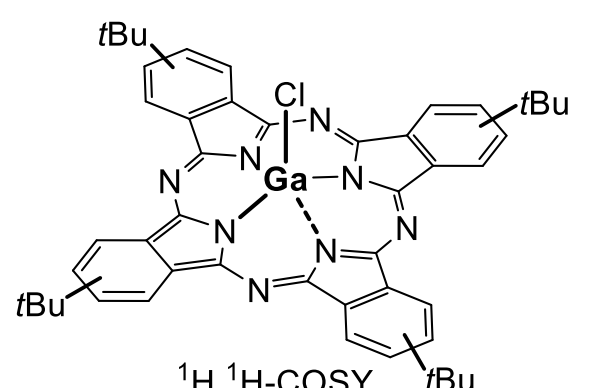

${ }^{1} \mathrm{H},{ }^{1} \mathrm{H}-\mathrm{COSY}{ }_{t \mathrm{Bu}}$ (300.1 MHz, $\mathrm{CD}_{2} \mathrm{Cl}_{2}$ )
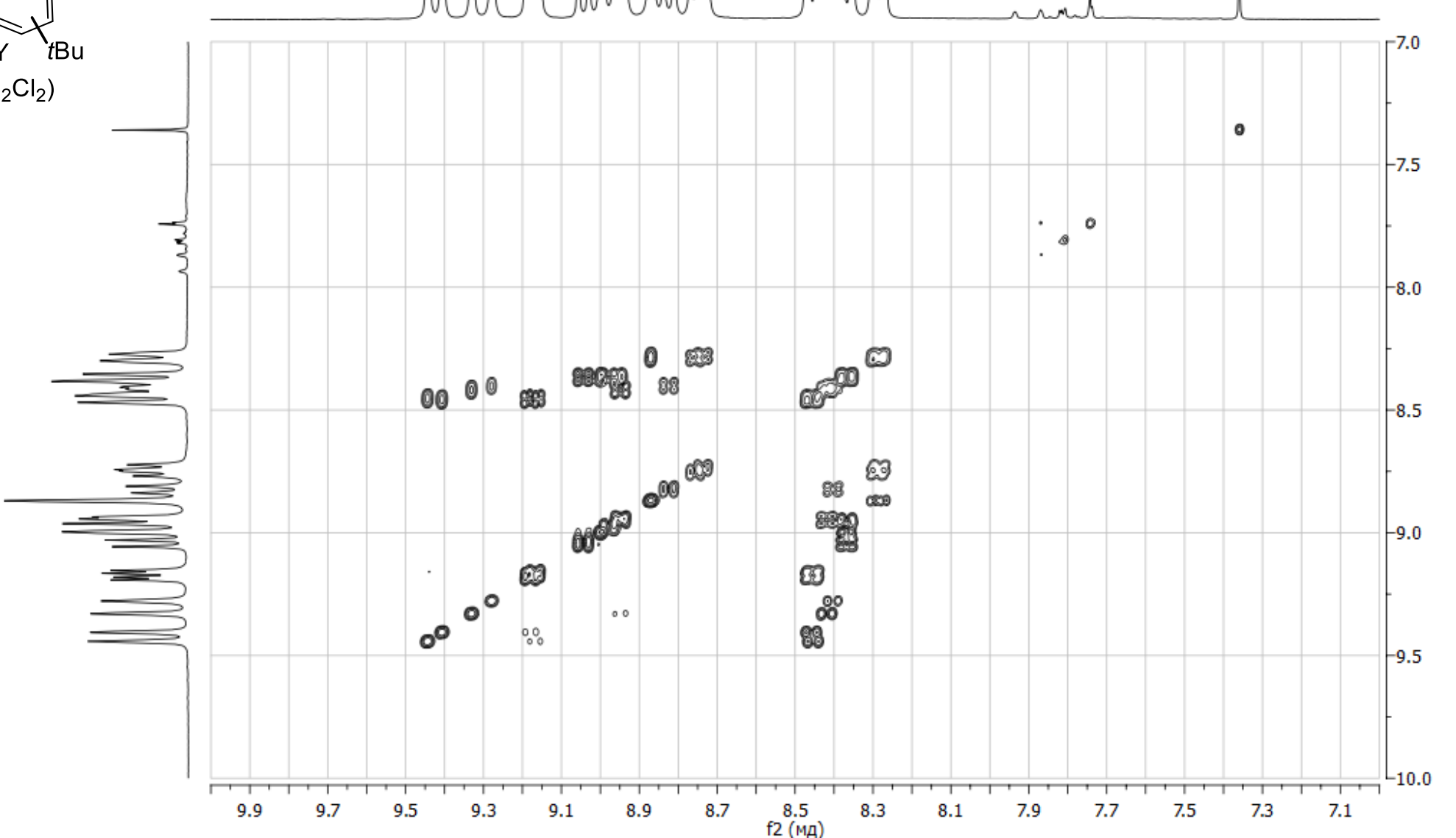
Figure S31.

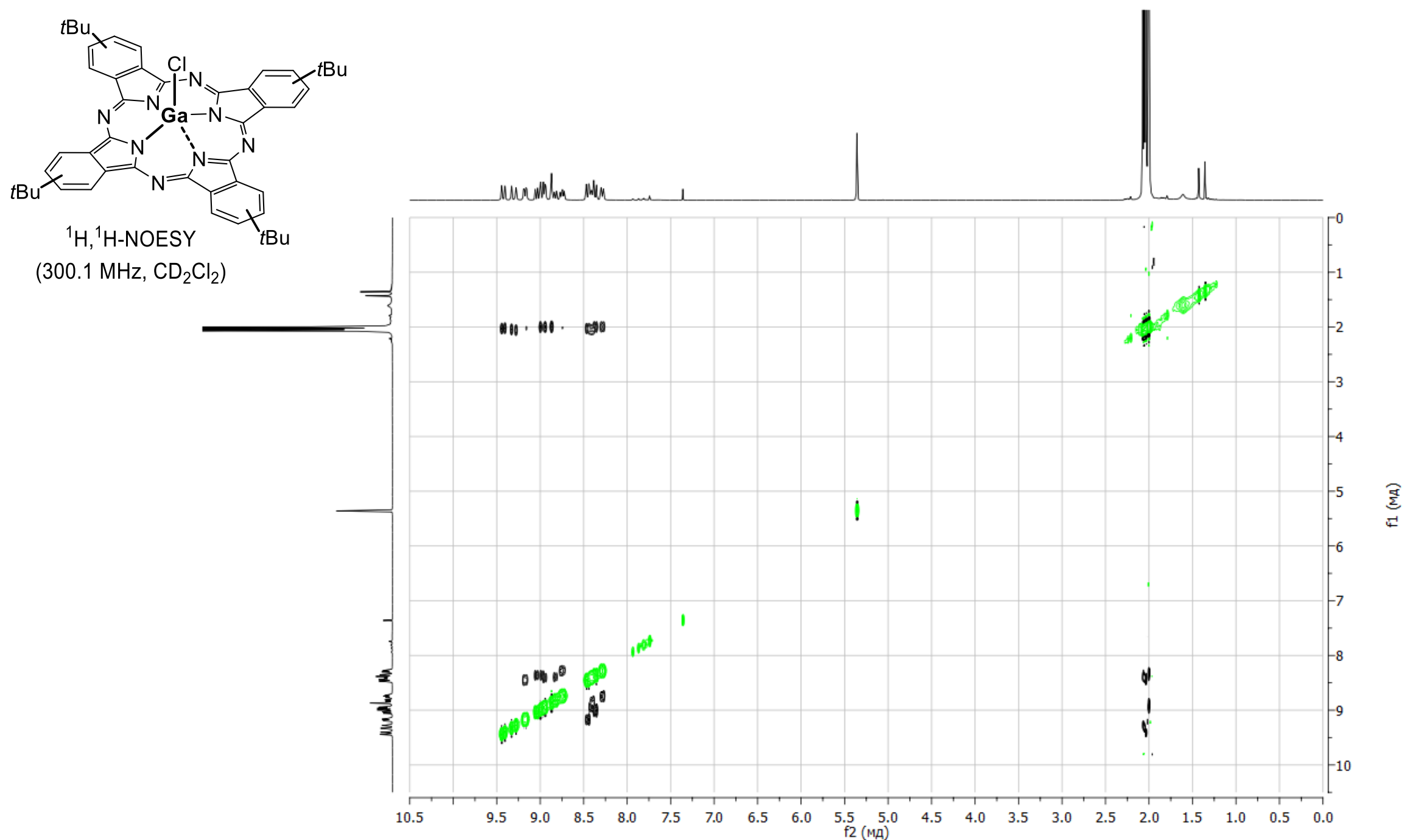


Figure S32.

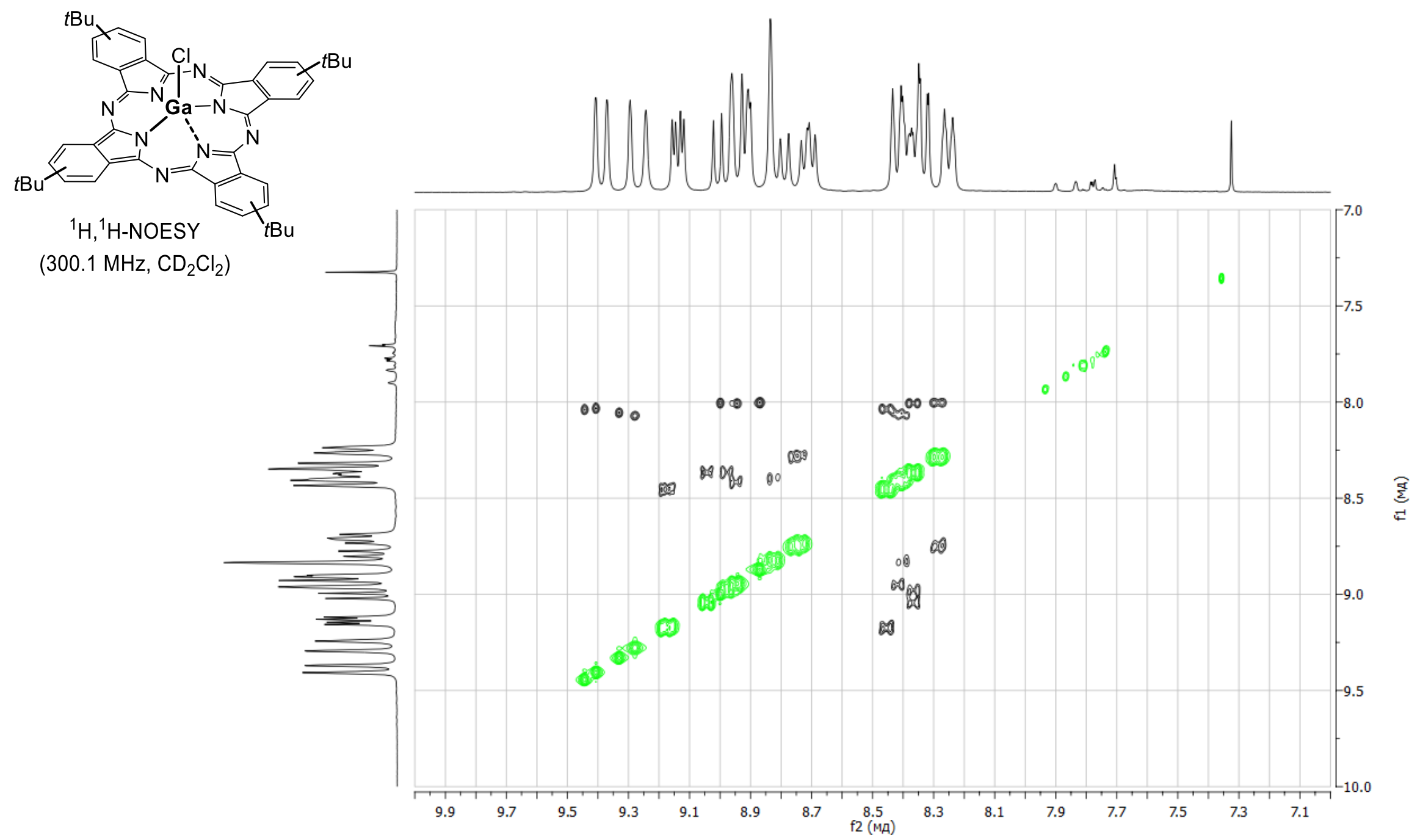


Figure S33.

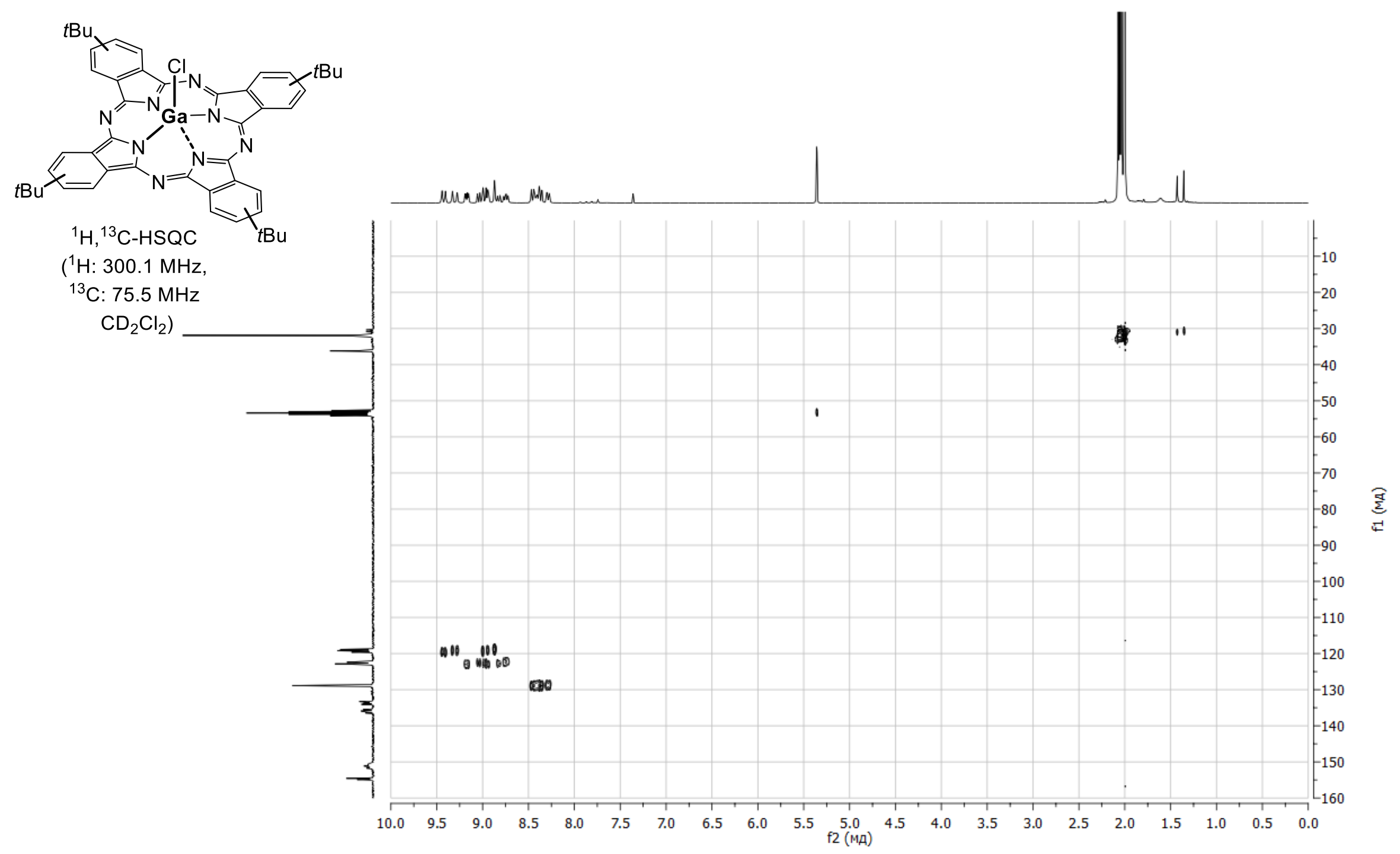


Figure S34.

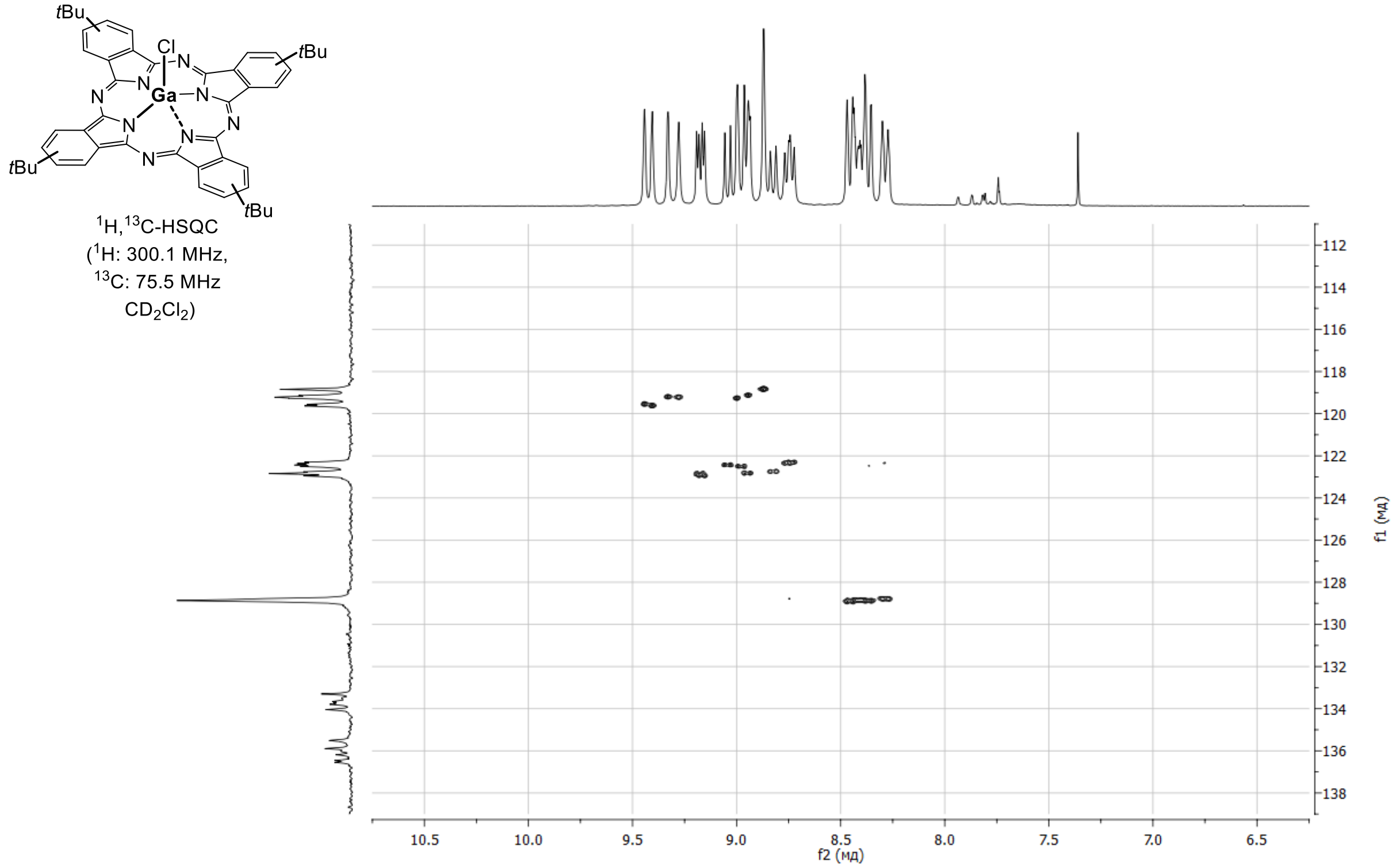


Figure S35.

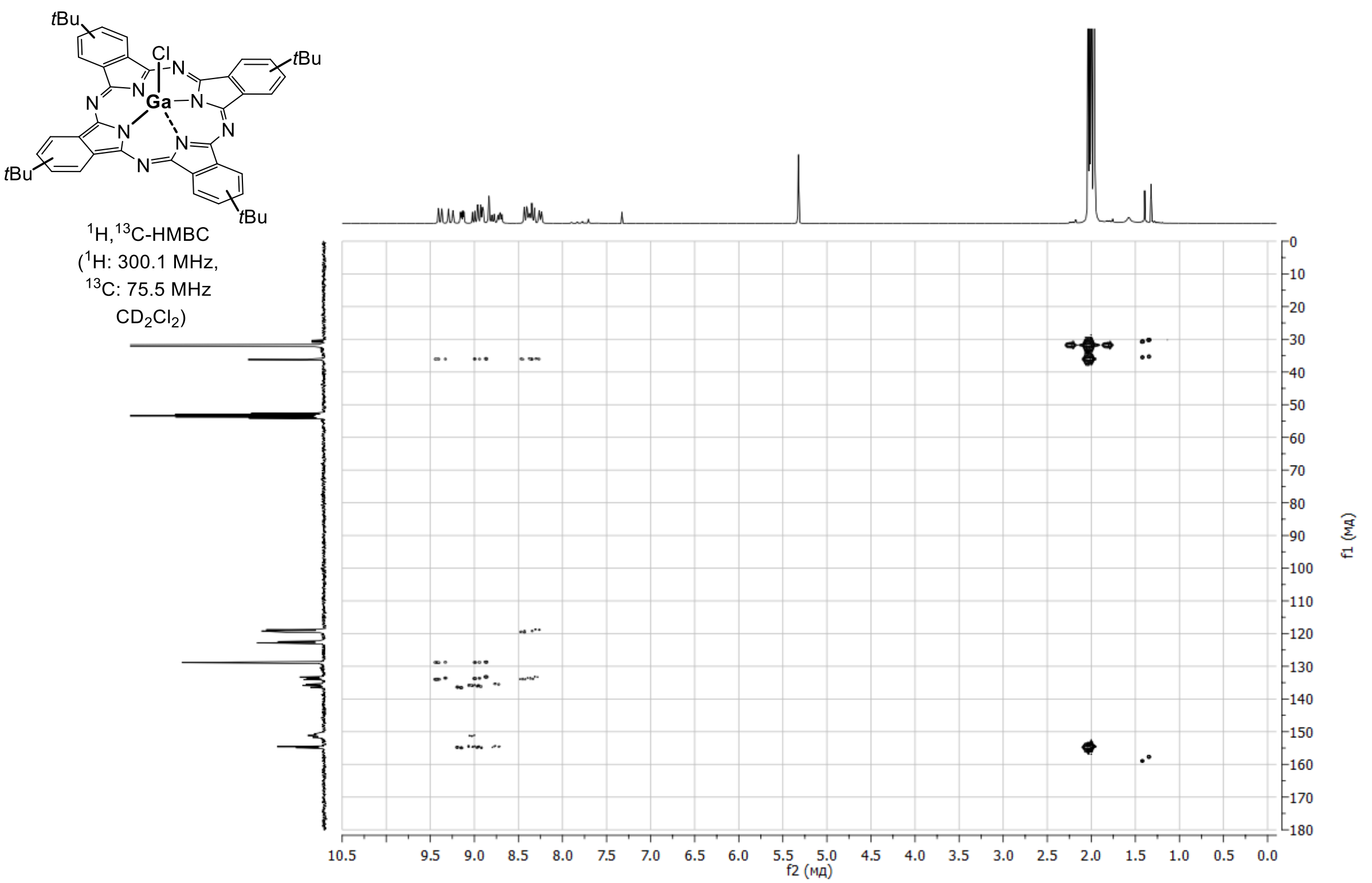


Figure S36.

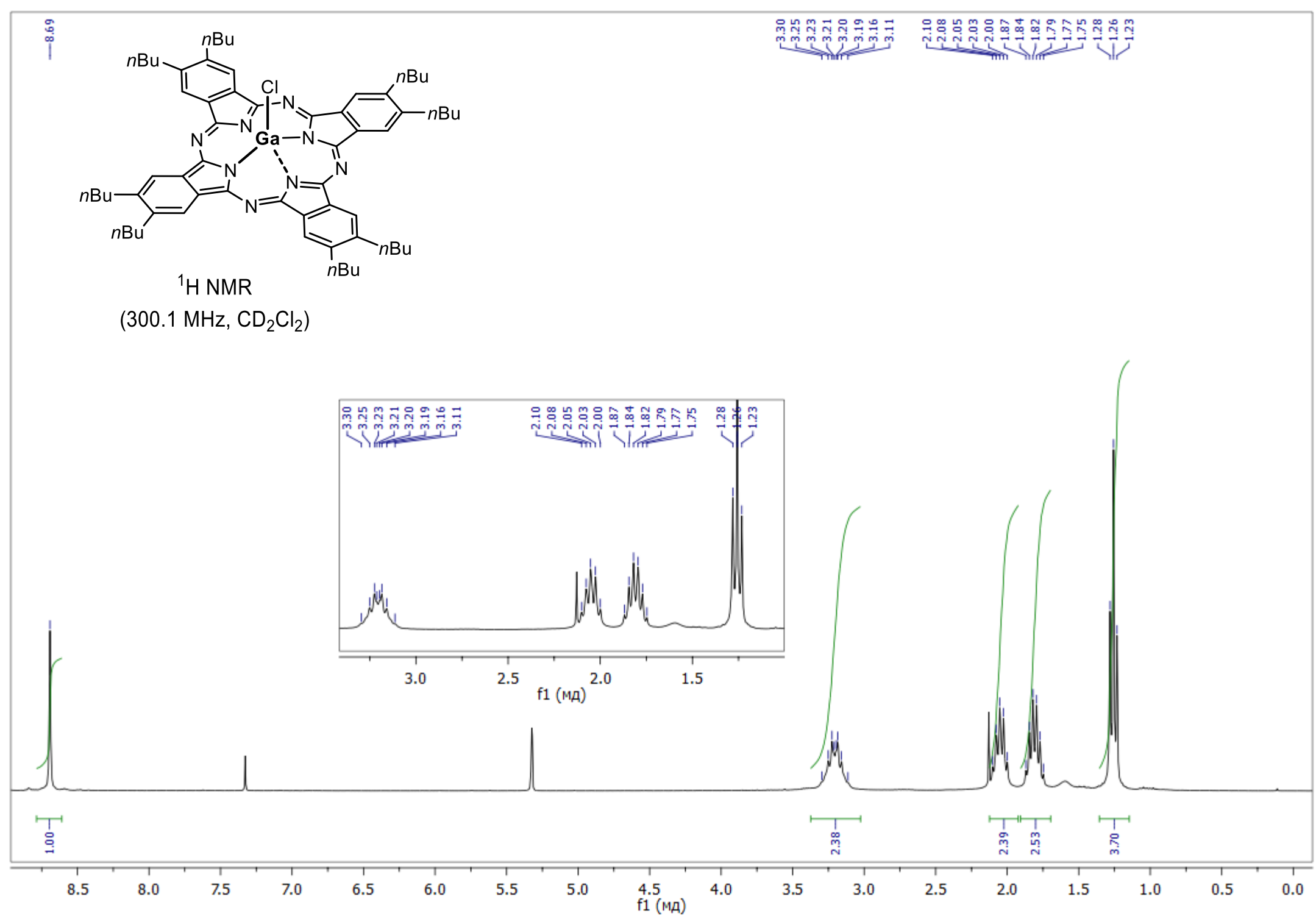


Figure S37.

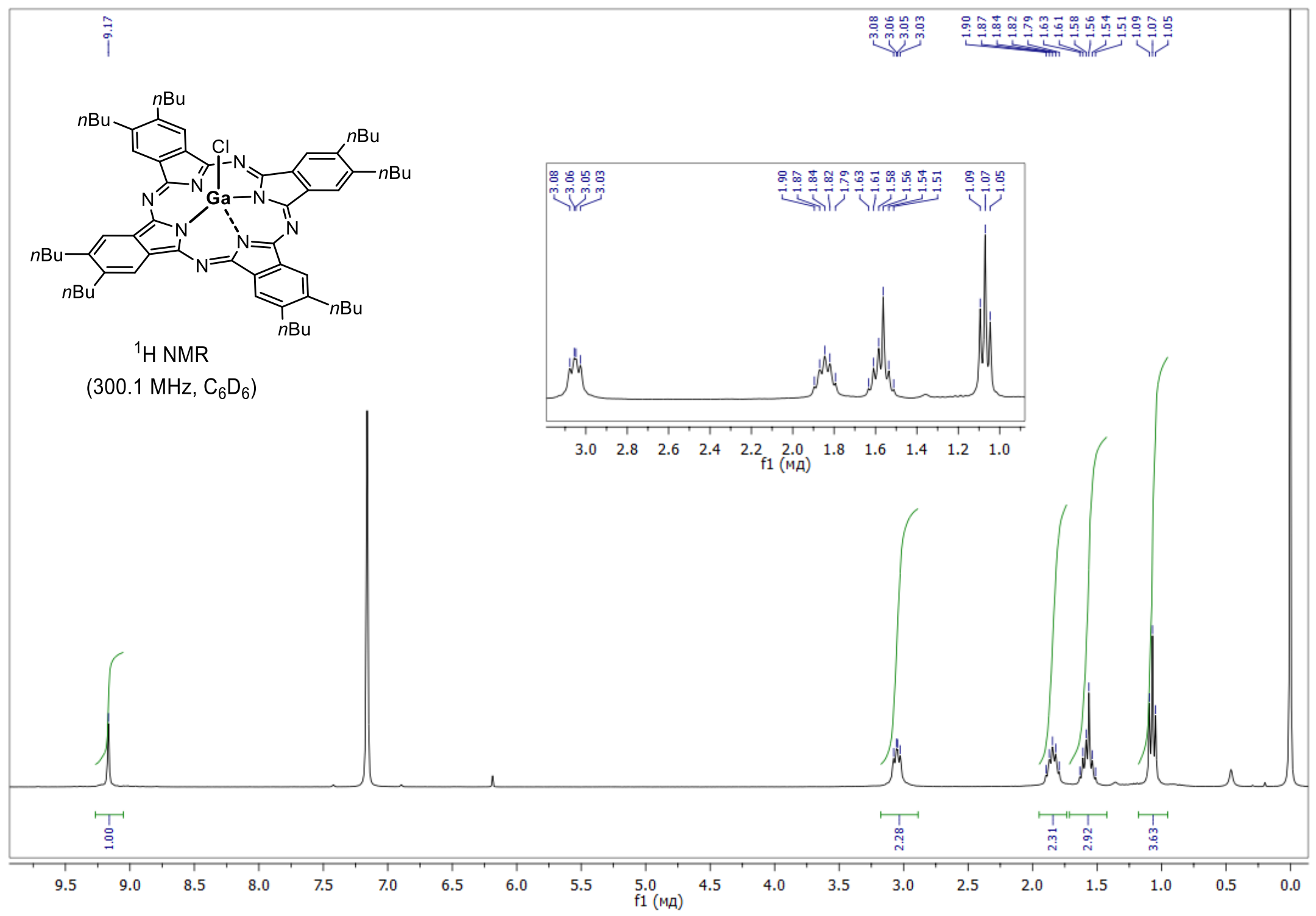


Figure S38.

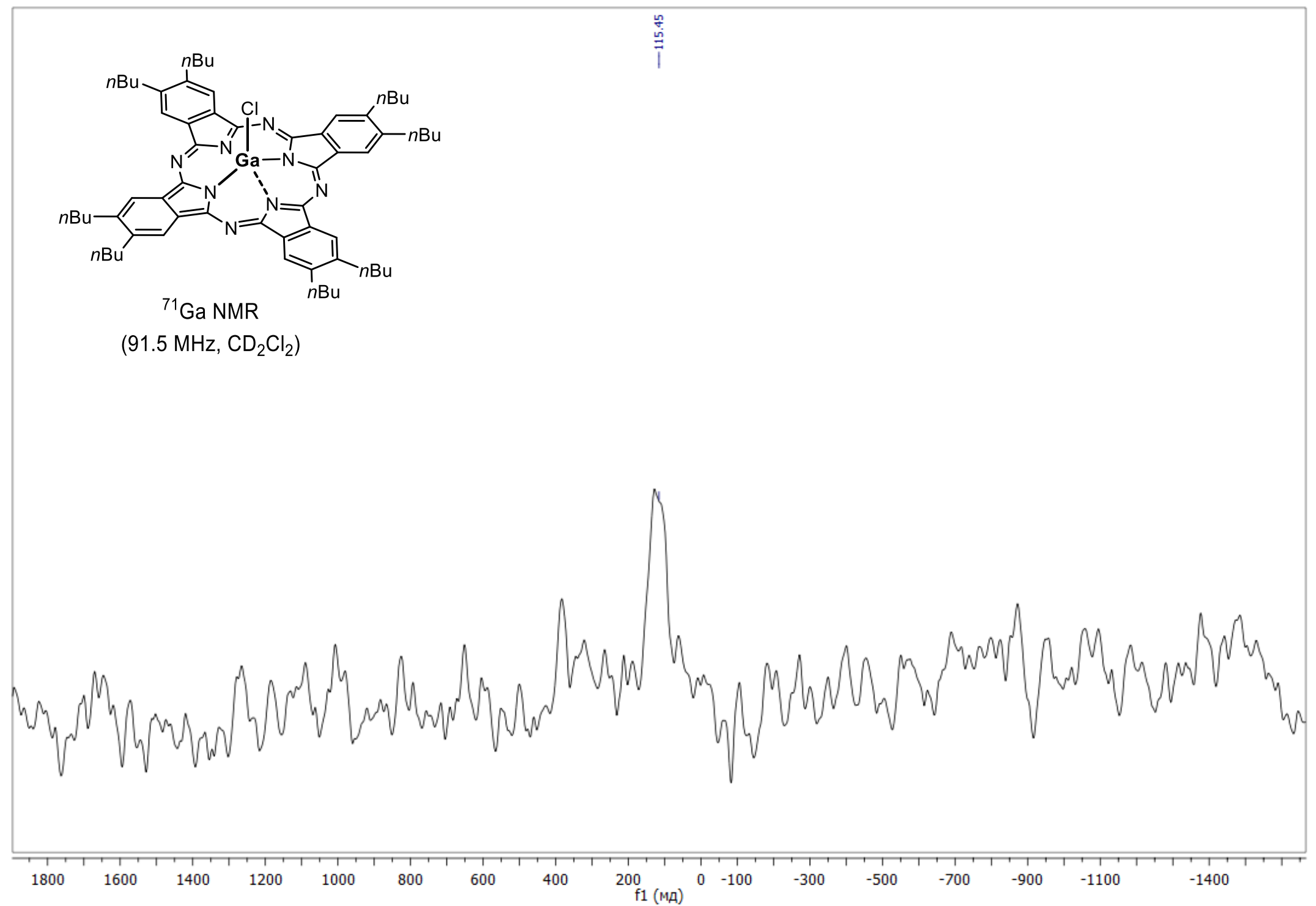




\section{Figure S39.}

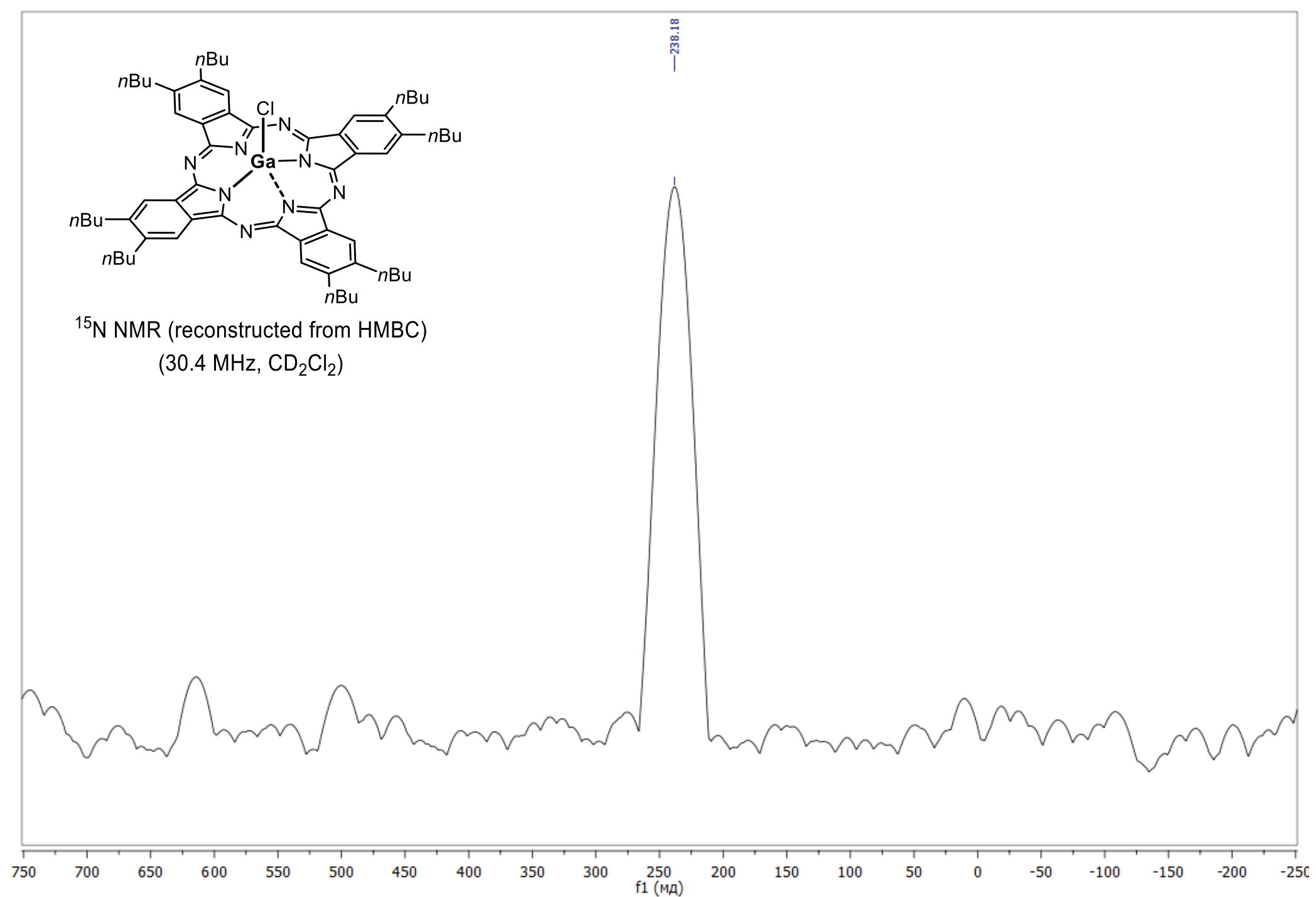


Figure S40.

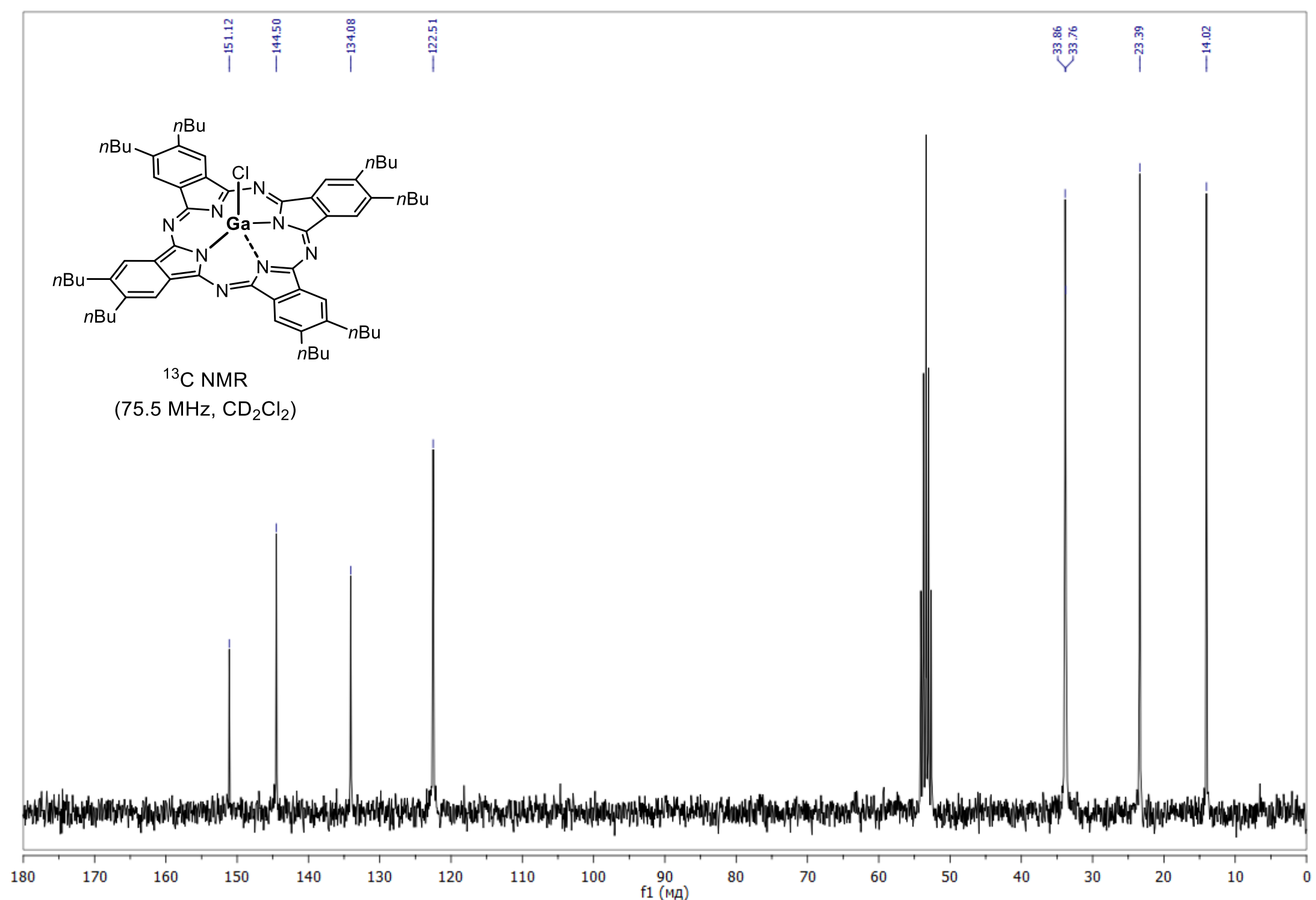




\section{Figure S41.}

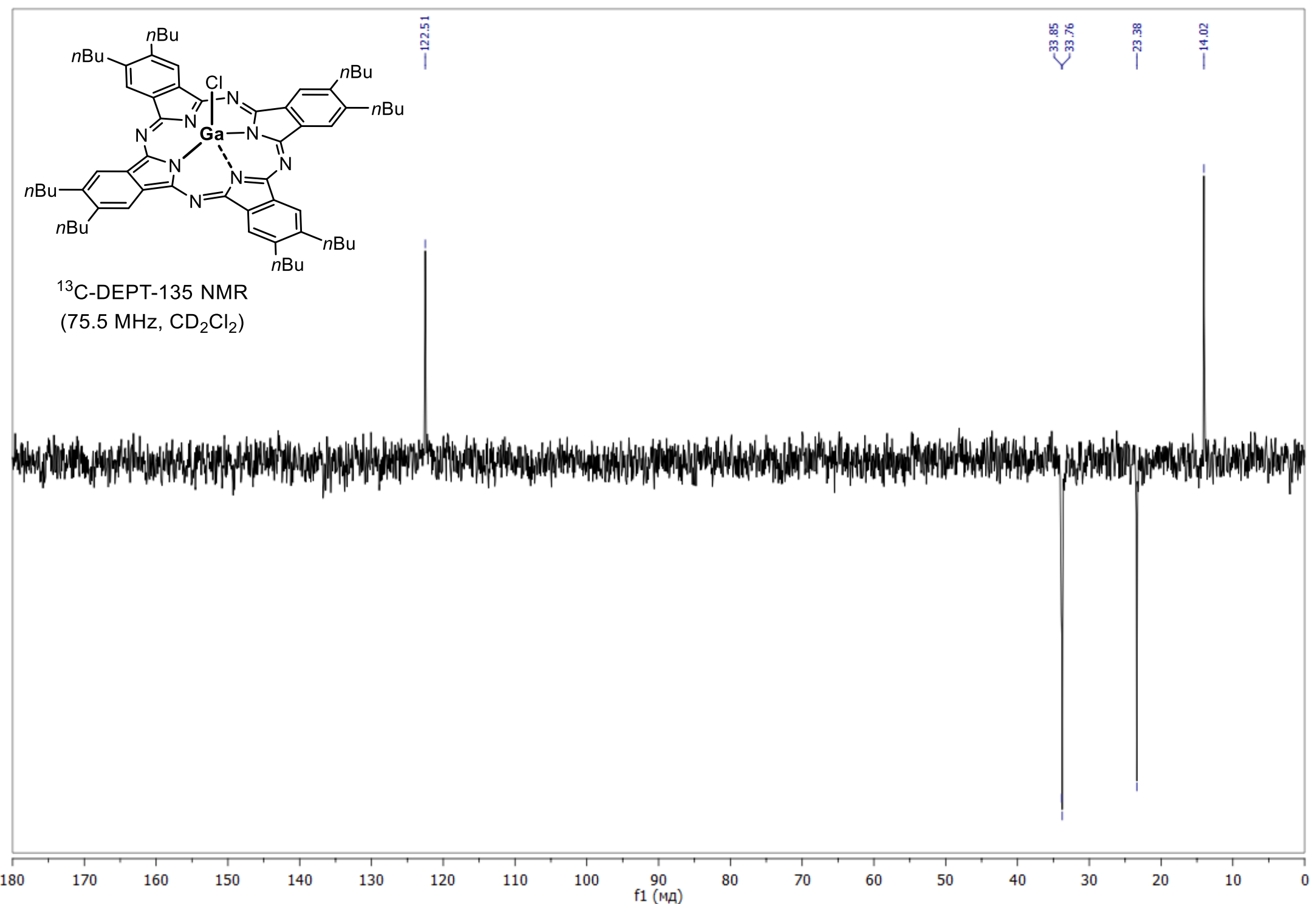


Figure S42.
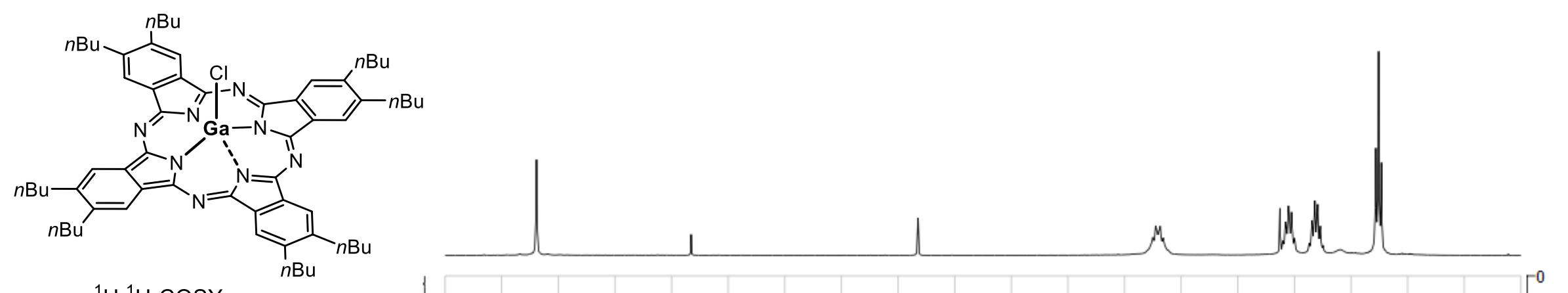

(300.1 MHz, $\mathrm{CD}_{2} \mathrm{Cl}_{2}$ )

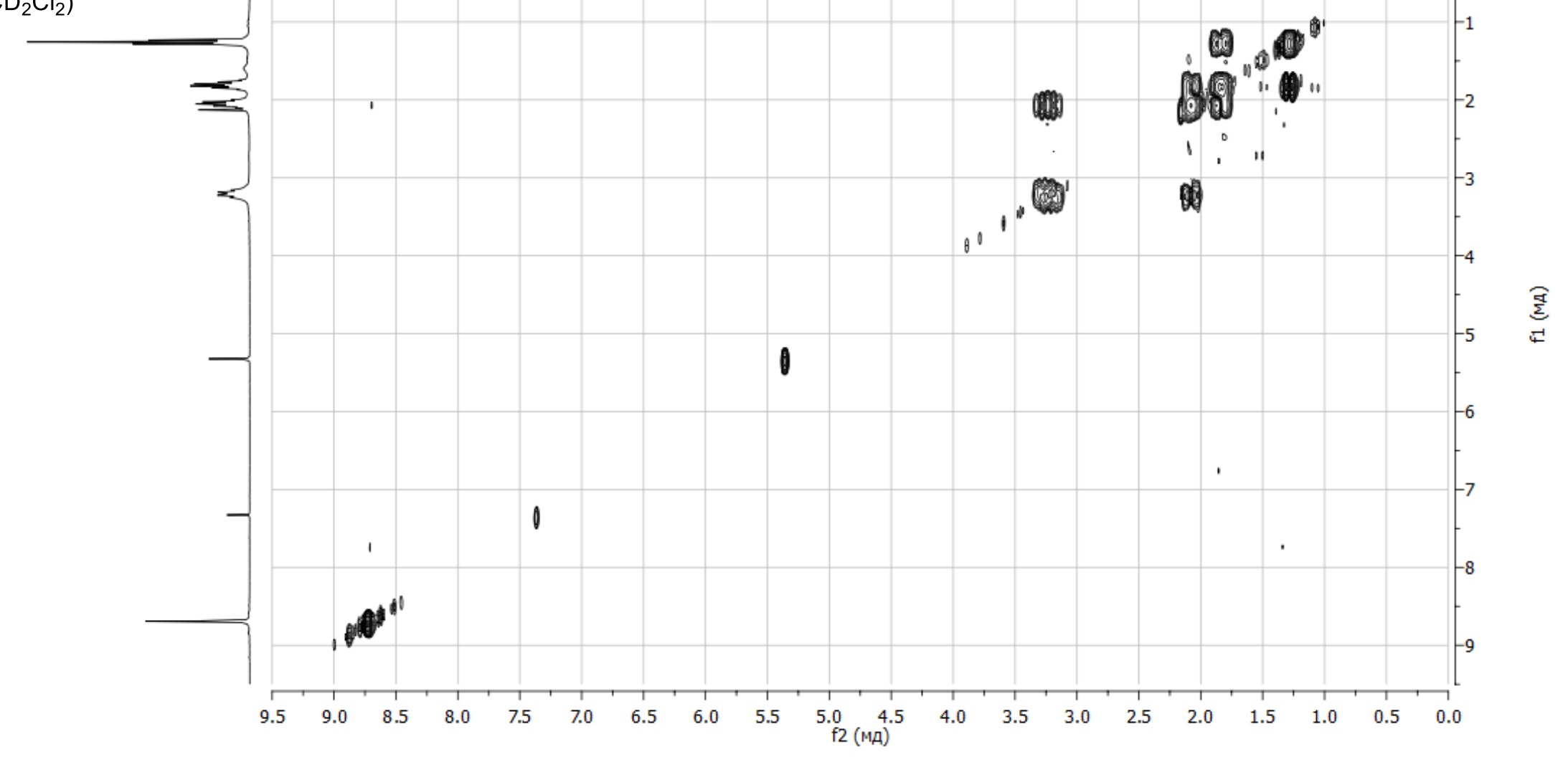


Figure S43.

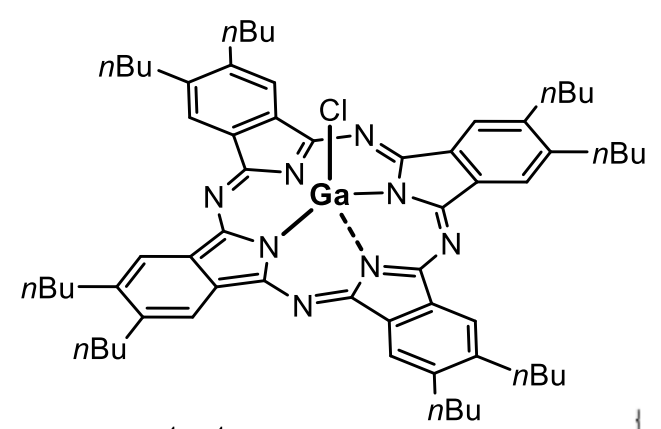

${ }^{1} \mathrm{H},{ }^{1} \mathrm{H}$-NOESY (300.1 MHz, $\mathrm{CD}_{2} \mathrm{Cl}_{2}$ )

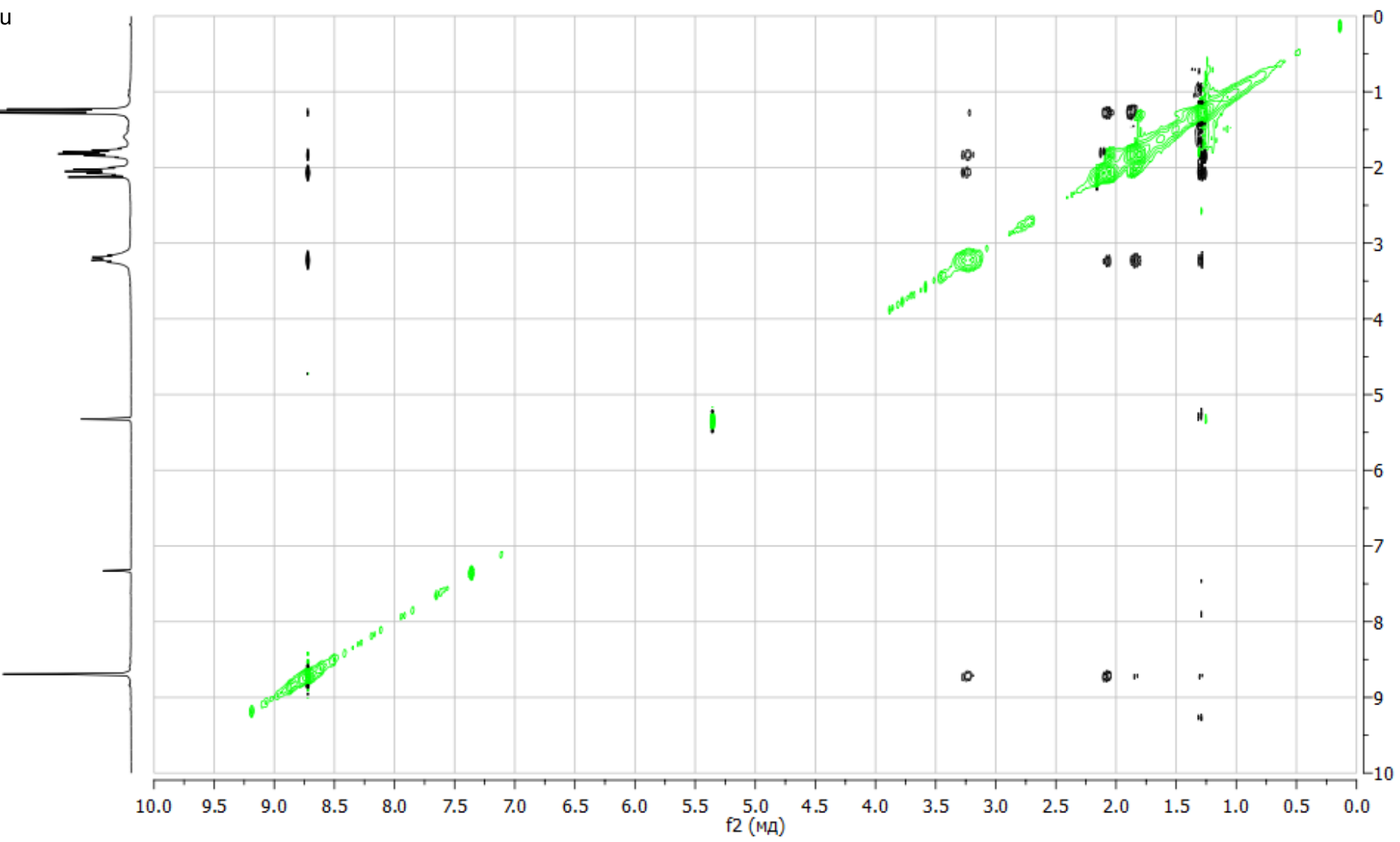


Figure S44.

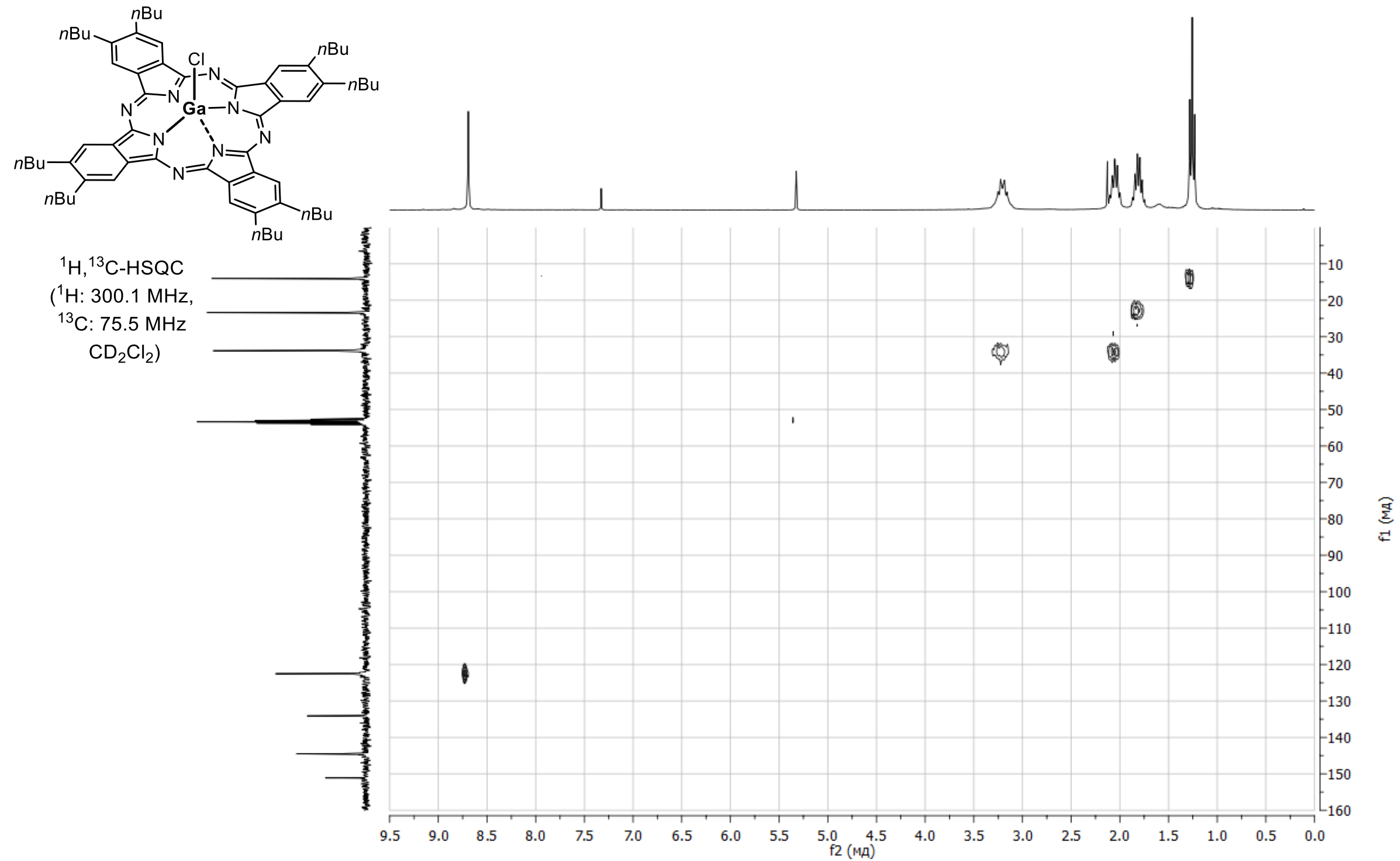


Figure S45.

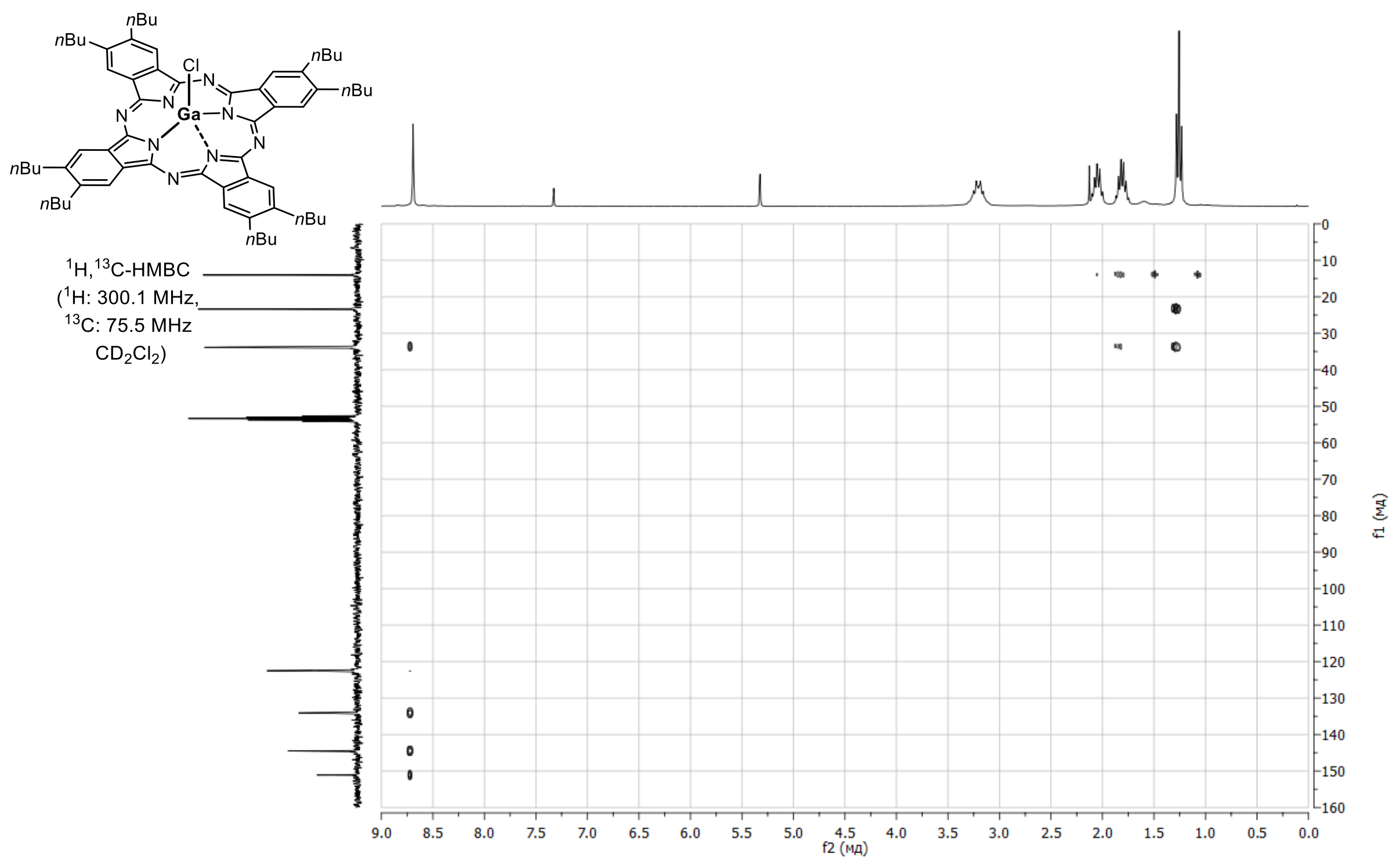


Figure S46.

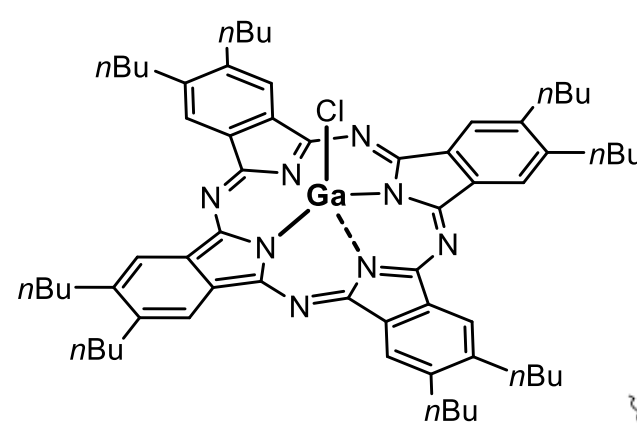

${ }^{1} \mathrm{H},{ }^{15} \mathrm{~N}-\mathrm{HMBC}$

$\left({ }^{1} \mathrm{H}: 300.1 \mathrm{MHz}\right.$,

${ }^{15} \mathrm{~N}: 30.4 \mathrm{MHz}$ $\mathrm{CD}_{2} \mathrm{Cl}_{2}$ )

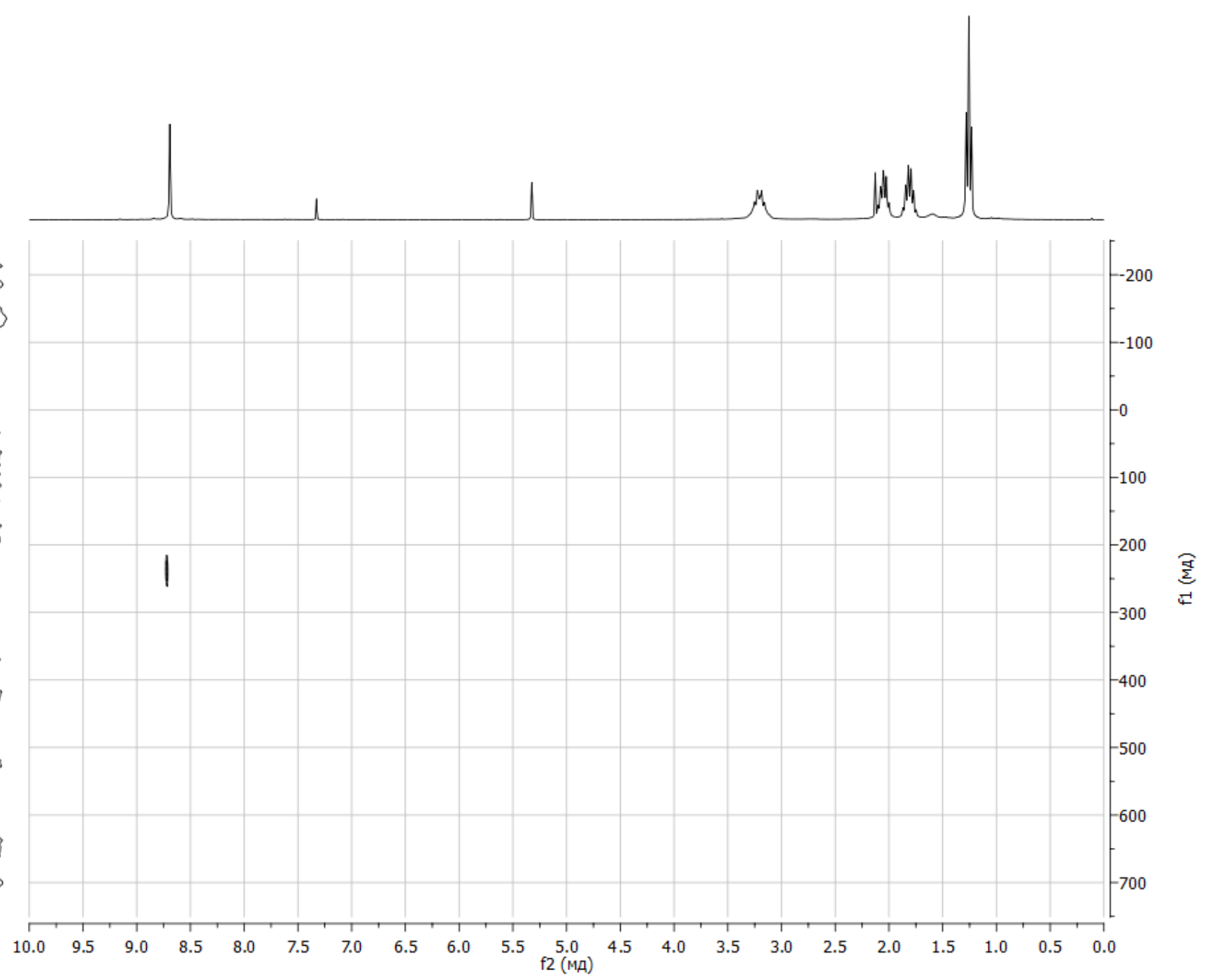


Figure S47.

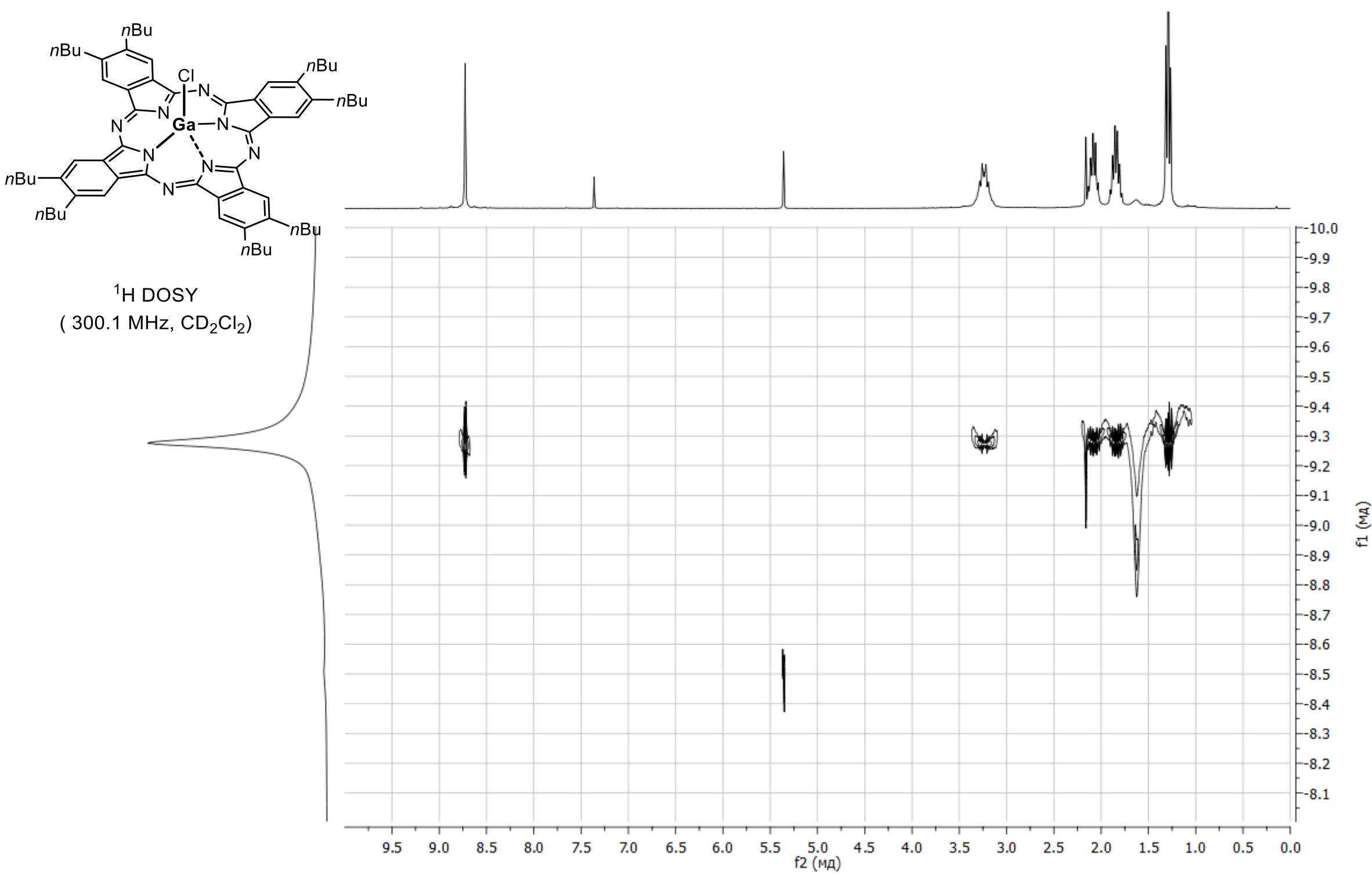


Figure S48.

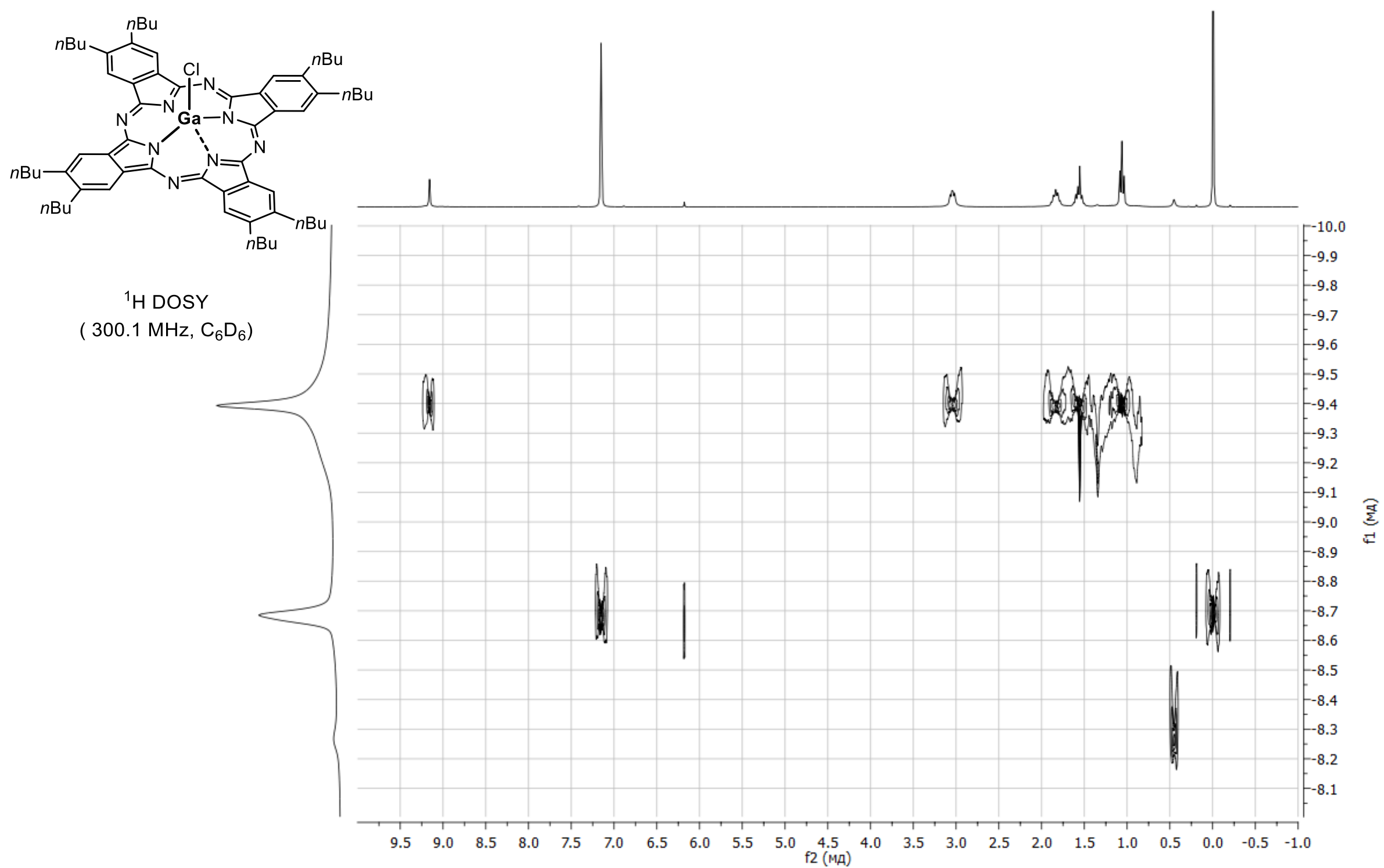


NMR spectra of indenes // Figure S49.

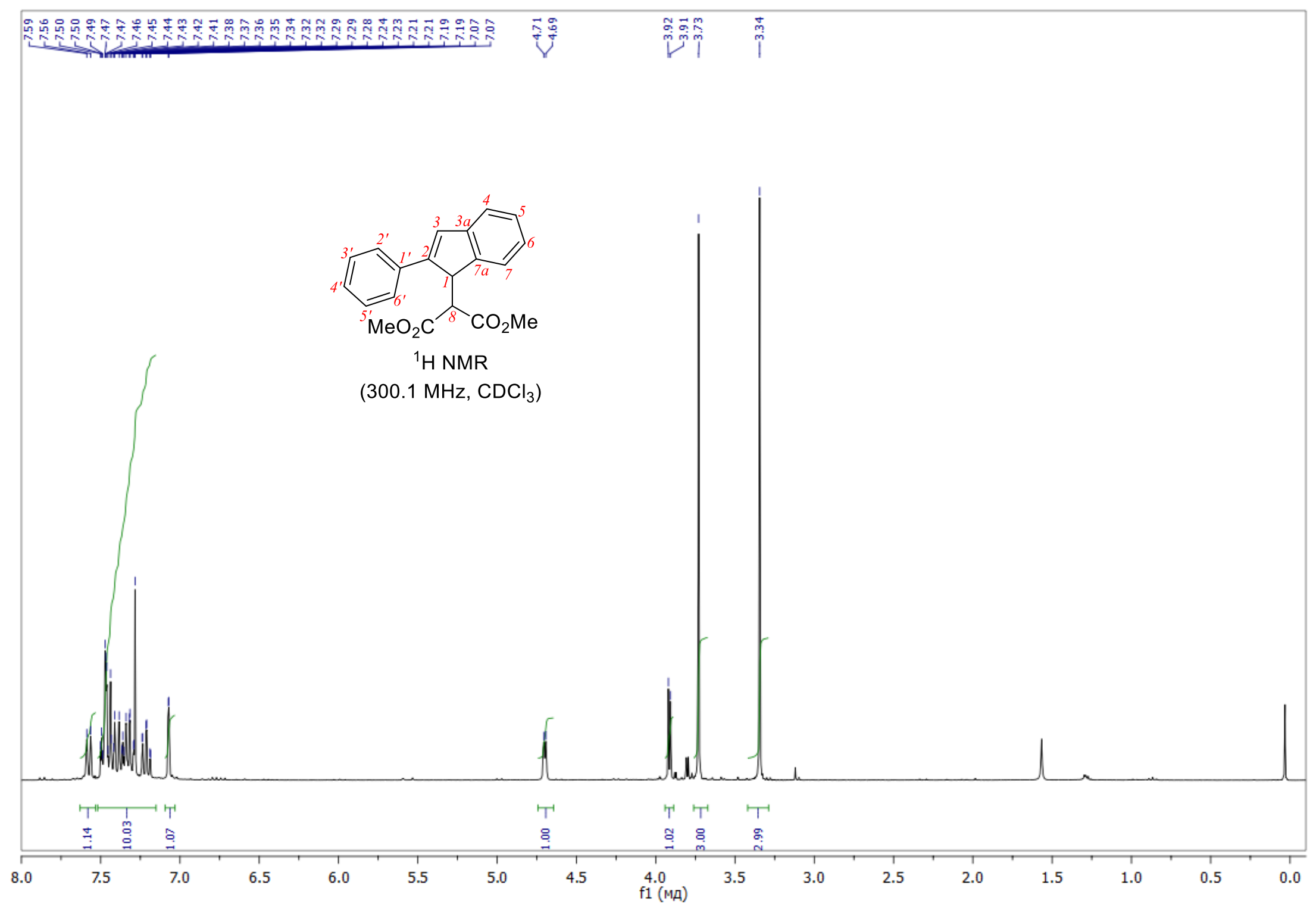


Figure S50.

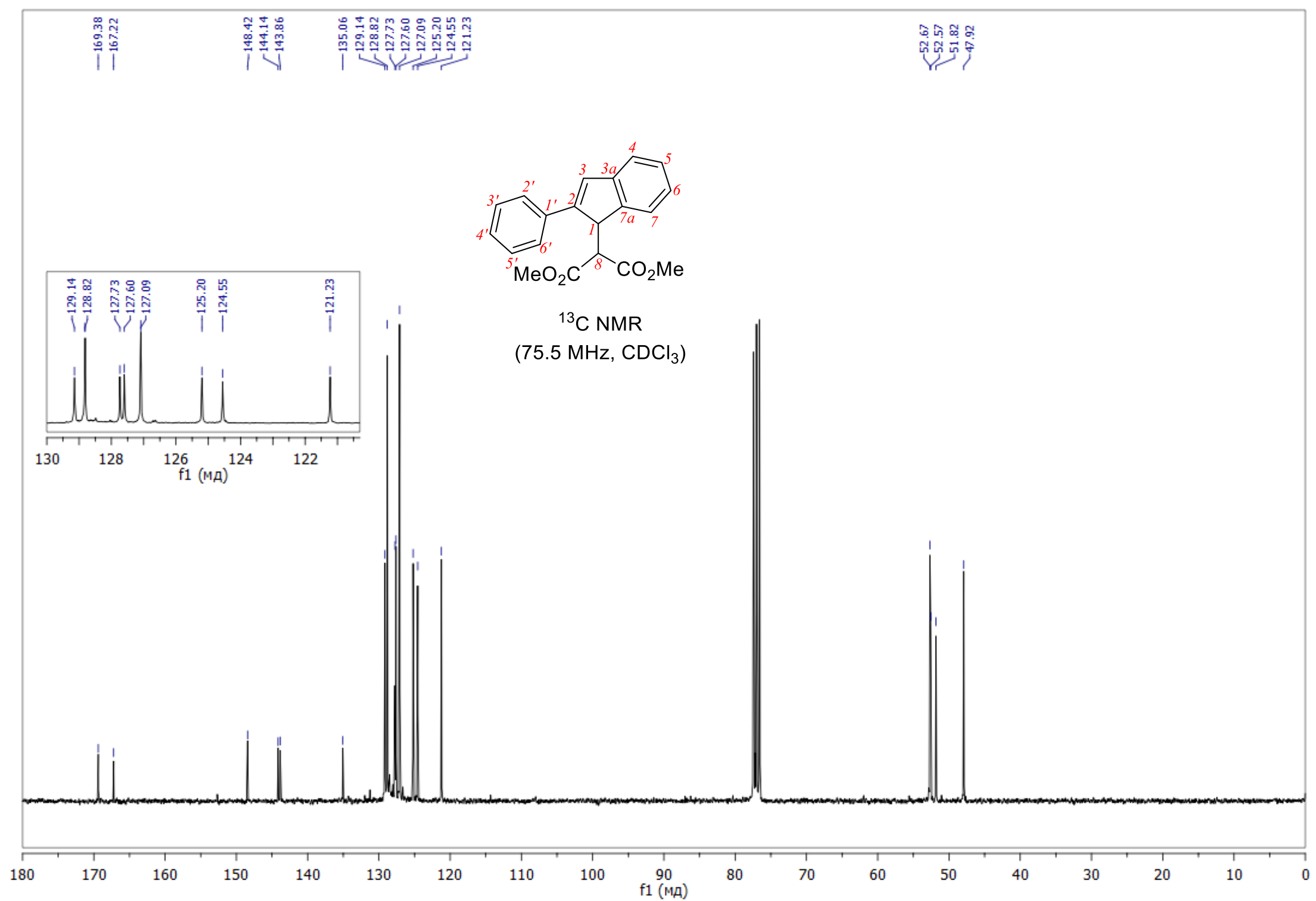


Figure S51.

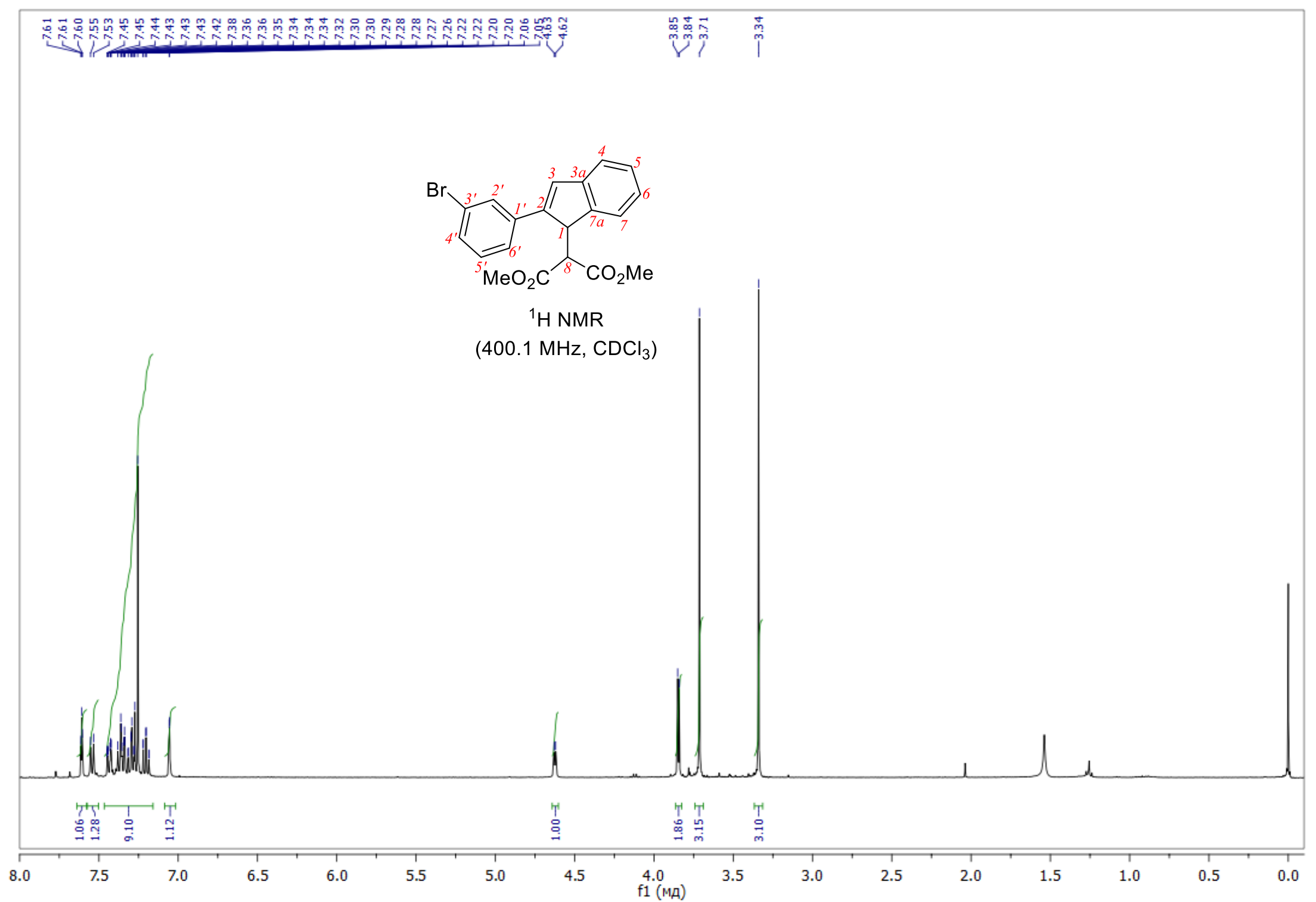


Figure S52.

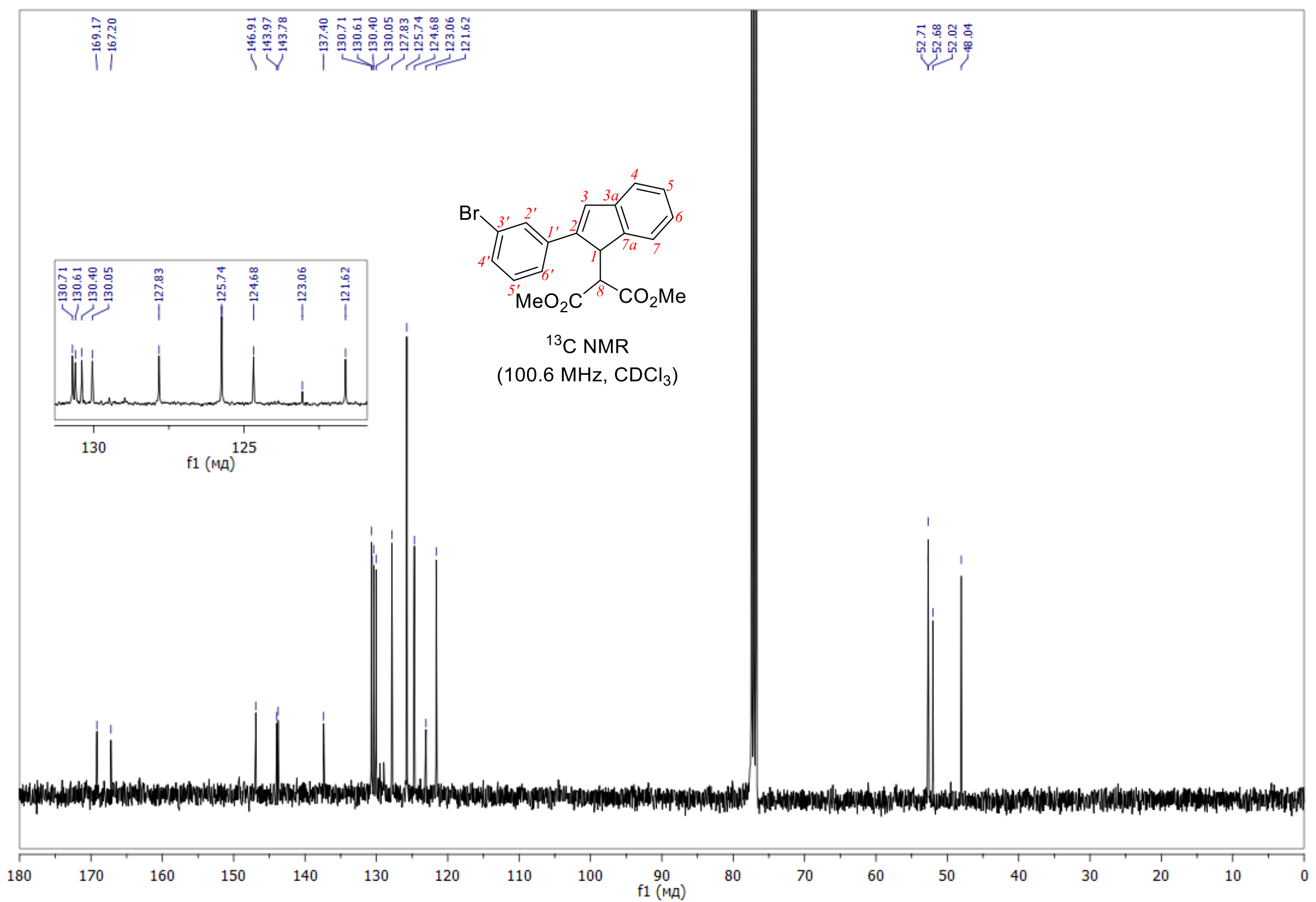


Figure S53.

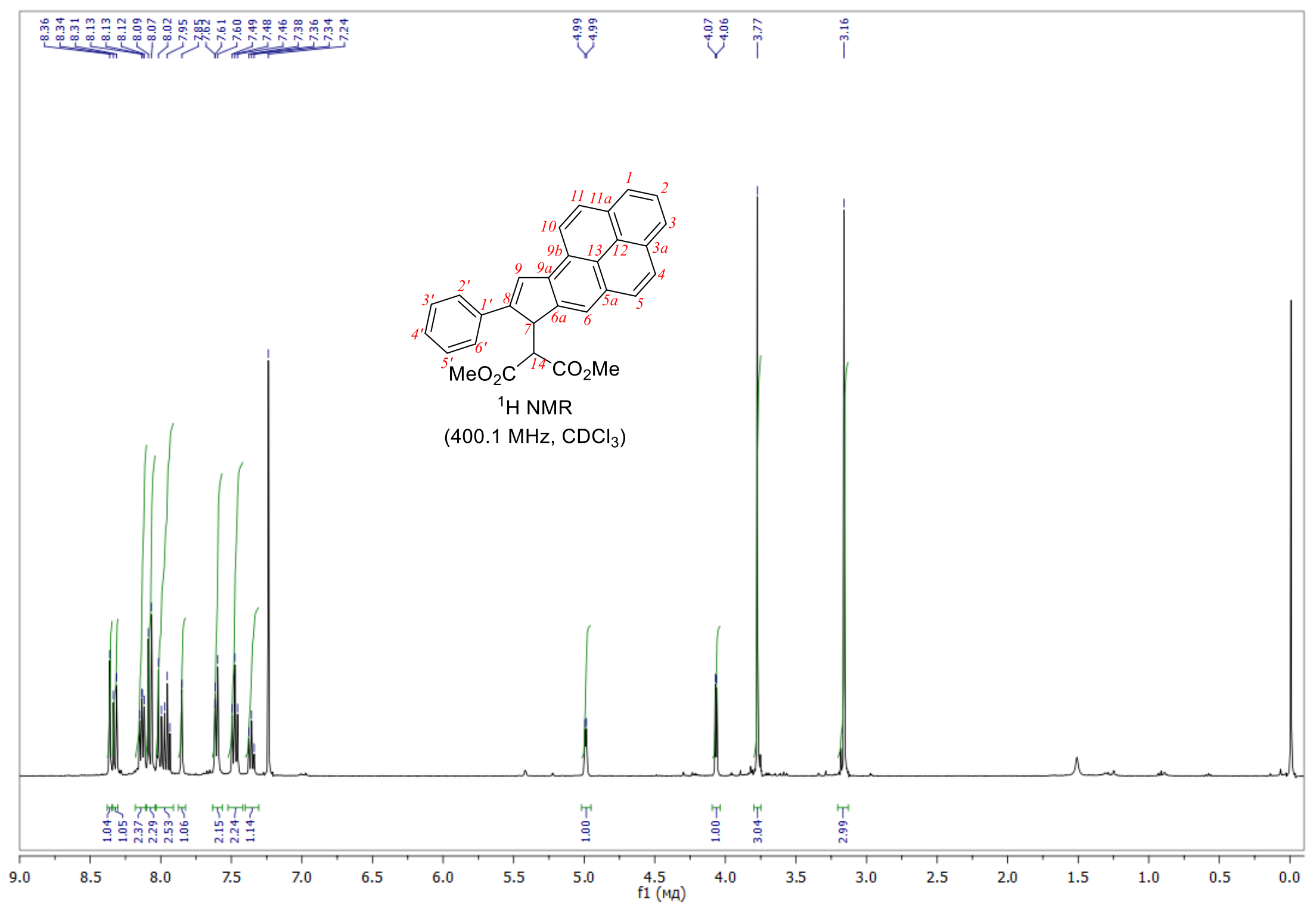


Figure S54.

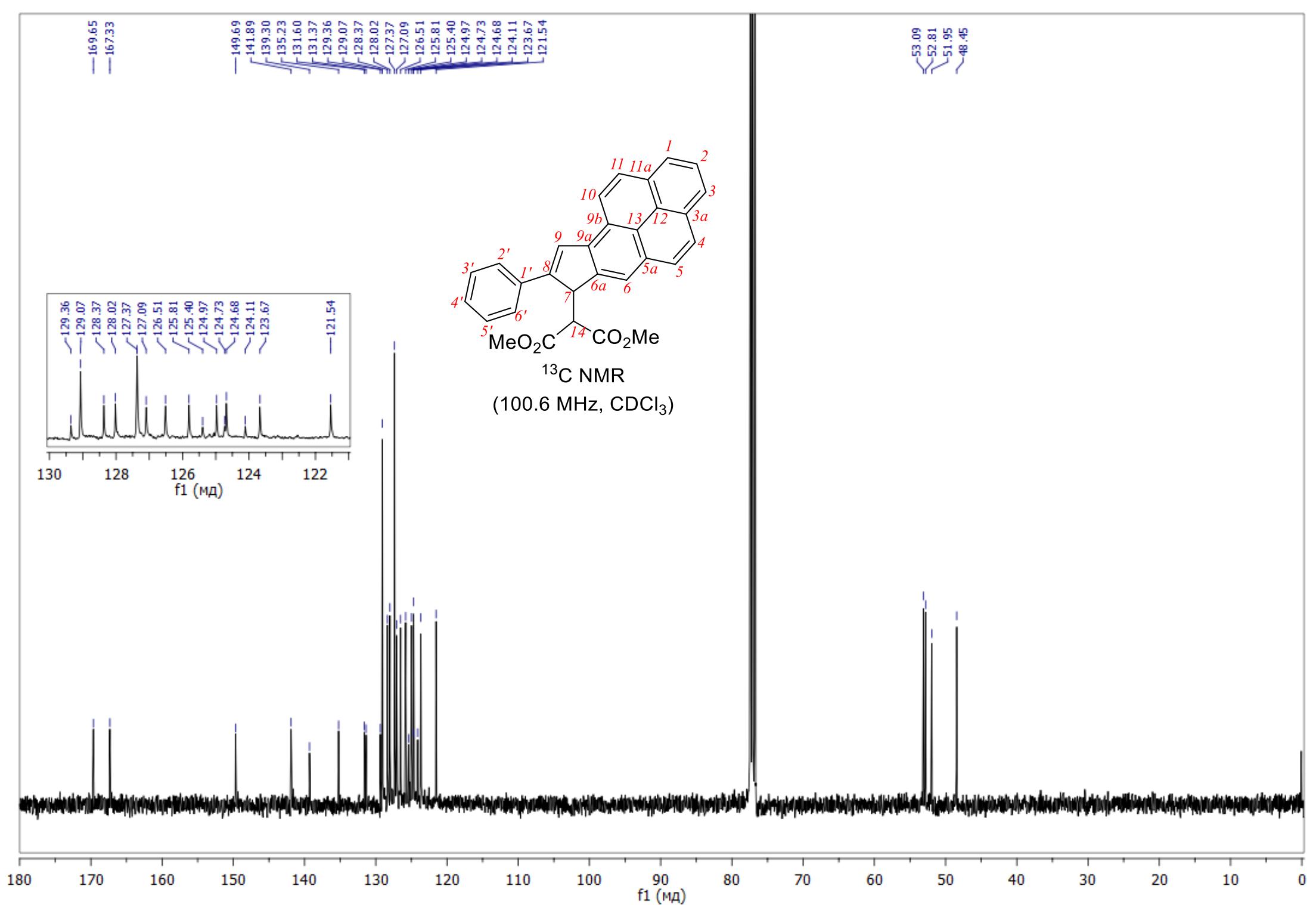


NMR spectra of tetrahydronaphthalene // Figure S55.

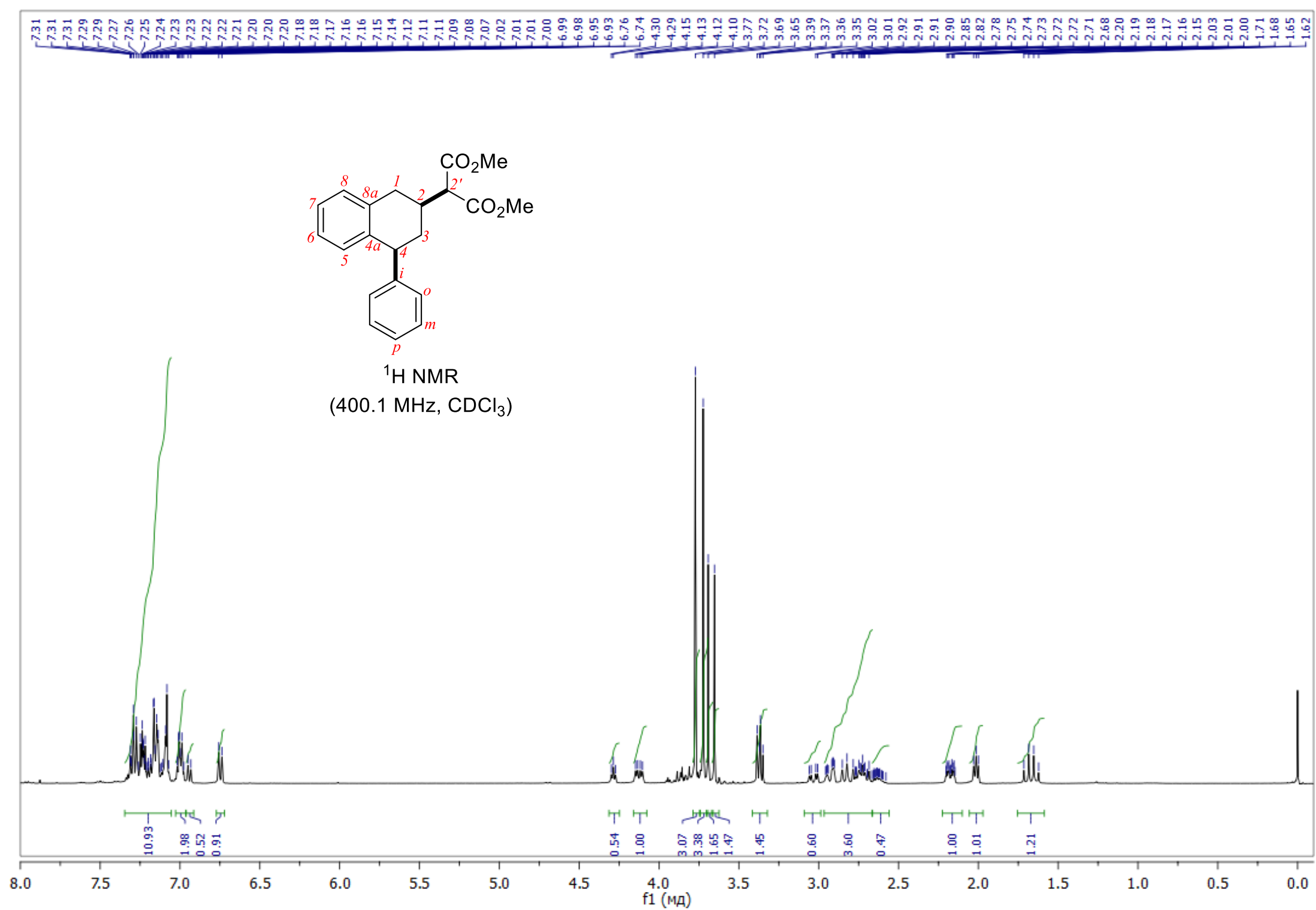


Figure S56.

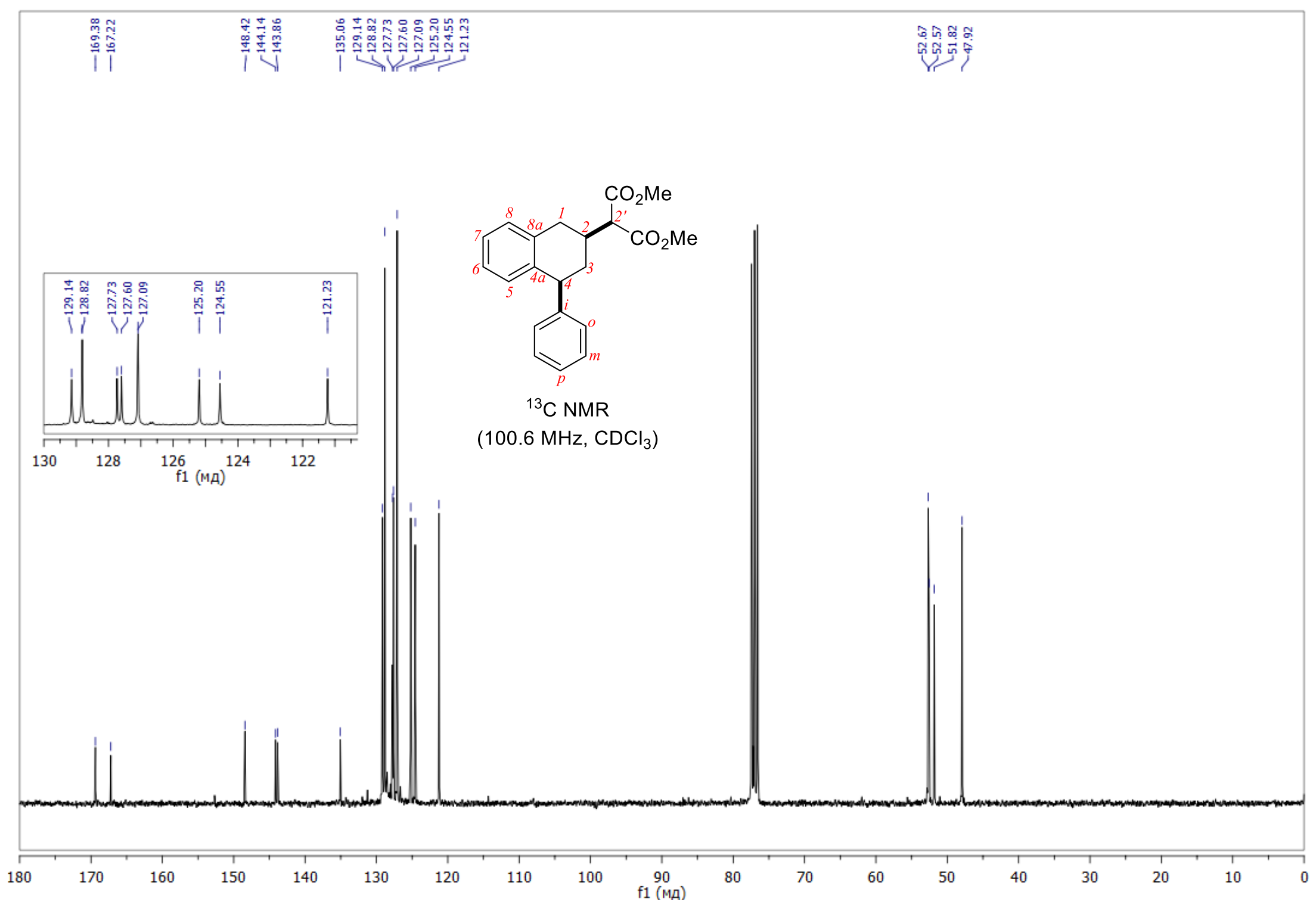

